\title{
Review Article \\ Electron Band Alignment at Interfaces of Semiconductors with Insulating Oxides: An Internal Photoemission Study
}

\author{
Valeri V. Afanas'ev \\ Laboratory of Semiconductor Physics, Department of Physics and Astronomy, University of Leuven, Celestijnenlaan 200D, \\ 3001 Leuven, Belgium
}

Correspondence should be addressed to Valeri V. Afanas'ev; valeri.afanasiev@fys.kuleuven.be

Received 19 June 2013; Accepted 27 October 2013; Published 13 February 2014

Academic Editor: Victor V. Moshchalkov

Copyright (C) 2014 Valeri V. Afanas'ev. This is an open access article distributed under the Creative Commons Attribution License, which permits unrestricted use, distribution, and reproduction in any medium, provided the original work is properly cited.

Evolution of the electron energy band alignment at interfaces between different semiconductors and wide-gap oxide insulators is examined using the internal photoemission spectroscopy, which is based on observations of optically-induced electron (or hole) transitions across the semiconductor/insulator barrier. Interfaces of various semiconductors ranging from the conventional silicon to the high-mobility Ge-based $\left(\mathrm{Ge}, \mathrm{Si}_{1-x} \mathrm{Ge}_{x}, \mathrm{Ge}_{1-x} \mathrm{Sn}_{x}\right)$ and $\mathrm{A}_{\mathrm{III}} \mathrm{B}_{\mathrm{V}}$ group ( $\mathrm{GaAs}, \mathrm{In}_{x} \mathrm{Ga}_{1-x} \mathrm{As}$, InAs, GaP, InP, GaSb, InSb) materials were studied revealing several general trends in the evolution of band offsets. It is found that in the oxides of metals with cation radii larger than $\approx 0.7 \AA$, the oxide valence band top remains nearly at the same energy $( \pm 0.2 \mathrm{eV})$ irrespective of the cation sort. Using this result, it becomes possible to predict the interface band alignment between oxides and semiconductors as well as between dissimilar insulating oxides on the basis of the oxide bandgap width which are also affected by crystallization. By contrast, oxides of light elements, for example, $\mathrm{Be}, \mathrm{Mg}, \mathrm{Al}, \mathrm{Si}$, and $\mathrm{Sc}$ exhibit significant shifts of the valence band top. General trends in band lineup variations caused by a change in the composition of semiconductor photoemission material are also revealed.

\section{Introduction}

Interfaces of semiconductors with wide-bandgap oxide insulators are encountered in a broad variety of electron devices including technologically important areas of logic, memory, and high-frequency integrated circuits. To meet the scaling requirements for the future generations of these electronic devices in terms of specific capacitance, gate leakage current, and carrier mobility in semiconductor channel, the search for suitable semiconductors and insulators capable of replacing the traditionally used $\mathrm{Si} / \mathrm{SiO}_{2}$ entity leads to combinations of more and more exotic materials [1-5]. Commonly, two parameters are considered to be of outmost importance when evaluating the potential of an insulator for applications as a gate dielectric in a transistor or a blocking layer in a chargestorage memory cell: the dielectric permittivity $(\kappa)$ and the bandgap width $\left(E_{g}\right)[1,6-10]$. However, the leakage current is determined by the rates of the electron and hole injection at the interfaces which, in turn, are sensitive to the conduction and valence band offsets, respectively, rather than to the insulator bandgap width per se [11]. The practical importance of interface barriers stimulated considerable efforts devoted to the analysis of the electrode effects on the interface band alignments. Up to this day, most of the interface barrier heights and band offsets have been calculated theoretically [12] because reproducible fabrication of metal/oxide and oxide/semiconductor entities of high quality represents a formidable processing challenge. However, the predicted band offset values appear to differ significantly (by $0.5 \mathrm{eV}$ or more) from the available experimental results which is probably caused by the application of inadequate models to simulate the interface structure. Furthermore, adding to this inconsistency, a large number of experimental studies have been conducted using an inappropriately oversimplified analysis of the photoelectron spectra which makes it worth discussing in more detail.

From the experimental point of view, the reliable quantification of the barrier height at the semiconductor/insulator or metal/insulator interfaces does not represent an easy task. The major difficulty is related to the fact that the frequently used 
photoelectron spectroscopy methods suffer from the attendant insulator charging effects [13-17]. The insulator charging artifacts may lead to a systematic error in the measured band offset value unless adequately corrected for [18-21].

In the present paper the recent results obtained by using spectroscopy of internal photoemission (IPE) and photoconductivity (PC) [21, 22] will be overviewed aiming at characterizing the barriers for electrons and holes at interfaces of silicon and various high-mobility semiconductors with a broad variety of insulating metal oxides. Four general issues are addressed: (1) the influence of the insulating oxide crystallinity on the interface band diagram as compared to an amorphous insulator; (2) the band offsets variations with the oxide bandgap width as a function of ionic radius $\left(r_{i}\right)$ of the oxide cation; (3) the effect of composition on the bandgap edge energies in high-mobility semiconductor alloys; and (4) the band alignment in semiconductor stacks. It is found that, in the case of compact cations $\left(r_{i} \lesssim 0.7 \AA\right)$ such as $\mathrm{Be}, \mathrm{Al}$, $\mathrm{Mg}$, and $\mathrm{Sc}$, the oxide crystallization results in widening of the gap, predominantly associated with the downshift of the O2pderived electron states at the top of the oxide valence band (VB). By contrast, in the case of larger cations $\left(r_{i} \gtrsim 0.7 \AA\right)$, no significant difference between the gaps of amorphous or (poly) crystalline oxides is found. The top of the VB preserves its energy in these oxides within a difference limit of about $\pm 0.2 \mathrm{eV}$, while the differences in bandgap are mostly reflected in the energy of the cation-derived electron states close to the bottom of the oxide conduction band (CB). This observation suggests that $\mathrm{O} 2 \mathrm{p}$-states at the $\mathrm{VB}$ top can be used as reliable reference level to evaluate the intrinsic band offsets between semiconductors and oxides as well as between two oxides if $r_{i}$ exceeds $0.7 \AA$. The comparison of energy barriers at interfaces between different semiconductors and oxide insulators indicates that the bulk density of electron states (DOS) represents the major factor determining the band alignment, with nearly no influence of the structure- or processingsensitive dipoles. In agreement with this conclusion, the band offsets are shown to follow the transitivity rule which opens the way to evaluate the band diagrams at interfaces between dissimilar semiconductors on the basis of IPE results concerning electron/hole barriers at the interfaces of these semiconductors with the same insulator, for instance, $\mathrm{Al}_{2} \mathrm{O}_{3}$.

Most of the results concerning the experimental determination of the band alignment presented in this work pertain to the interfaces of bulk-liked semiconductors. These results provide the frame of reference for future evaluation of other physical effects which may affect the electronic structure of solids such as strain, doping, or quantum confinement of electron states. For example, significant size-dependent variation of electronic structure is found in silicon nanoparticles embedded into an insulating matrix [23-25] which makes it different from the band alignment observed at interfaces of bulk silicon with the same insulators. Up to this day, only a limited amount of experimental information regarding band alignment at interfaces of nanoparticles is available which precludes one from a systematic analysis of these effects. Nevertheless, this area of research is firmly set for development in the near future.

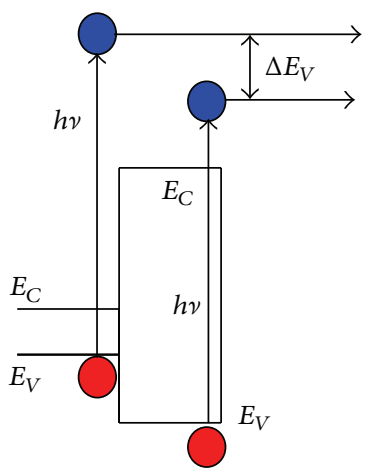

(a)

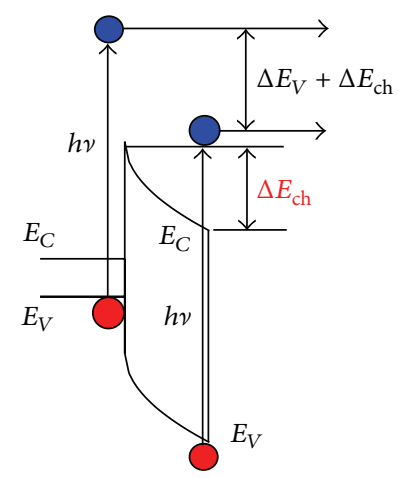

(b)
FIGURE 1: Schematic of the VB offset $\left(\Delta E_{V}\right)$ determination from an external photoemission experiment. While in the ideal case of the uncharged insulator (panel (a)) the energy difference between electrons emitted from the VB tops of the semiconductor and insulator exactly corresponds to the VB offset, the X-ray induced charging of the insulator (panel (b)) introduces an energy shift $\Delta E_{\mathrm{ch}}$ due to variation of the electrostatic potential across the insulating layer leading to a systematic error in the measured band offset value.

\section{Band Offsets Determination Using Spectroscopy of Internal Photoemission}

Experimentally, the band alignment at semiconductor interfaces can be determined in several ways by observing transport of electrons or holes across the interface. In the case of interfaces with insulators, however, application of the classical barrier characterization methods faces a major difficulty: at room temperature the equilibrium rate of electron (or hole) injection becomes negligible if the corresponding interface barrier height exceeds $2 \mathrm{eV}$. Under these circumstances, only charge carriers excited to the energy sufficient to surmount the energy barrier can be transported across the interface mandating the use of external excitation. Furthermore, if the insulating layer is subjected to charge injection or exposed to exciting UV-radiation, X-rays, electron, or particle beams, the traps usually encountered in wide-gap oxides will accumulate charge leading to a global shift of the energy levels in the insulator as compared to the uncharged initial state $[13,14]$, sometimes referred to as "the differential charging effect" [15]. This phenomenon is illustrated in Figure 1 which schematically depicts the energy band diagram of a semiconductor/insulator interface prior to (a) and after trapping of a positive charge in the bulk of the insulator (b). In theory, in a neutral sample (panel (a)) the VB offset can be measured by observing simultaneously the energy distribution of electrons emitted from the VBs of the semiconductor and insulator or by comparing them to two core level energies (the Kraut's method [26, 27]). However, in the presence of the oxide charge (panel (b)), all electron states in the outer layer of the insulator will be shifted in energy by $\Delta E_{\text {ch }}$ because of the electric field induced by the trapped charge. Taking into account that electron states located close to the surface of the sample provide the dominant contribution to electron photoemission, the charging results in a global electrostatic 


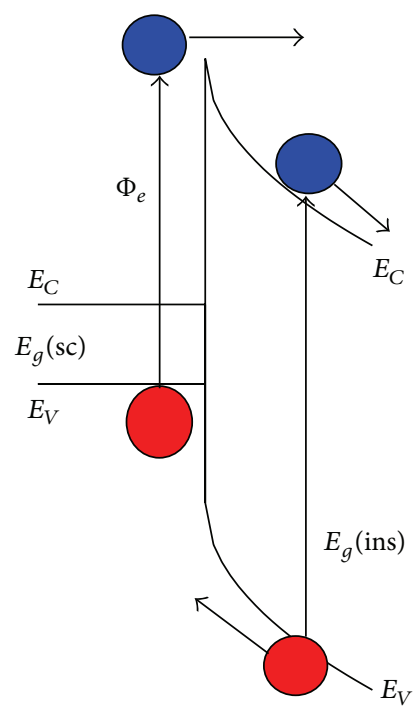

(a)

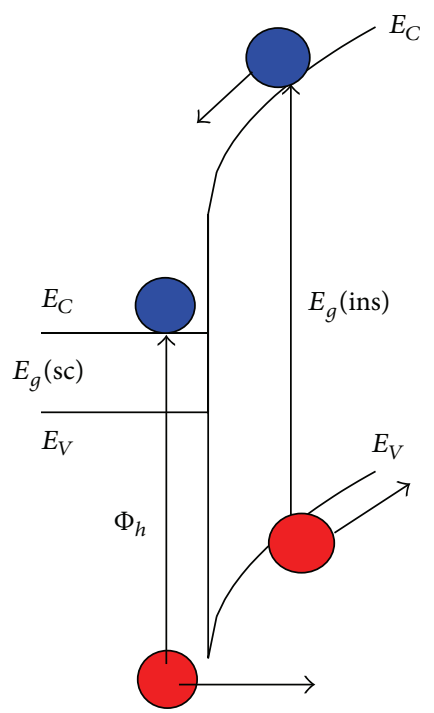

(b)

FIGURE 2: Schematic semiconductor/insulator energy band diagram for positive (a) and negative (b) bias applied to the top metal electrode (not shown), indicating the electron transitions in the case of electron IPE (threshold $\Phi_{e}$ ) and hole IPE (threshold $\Phi_{h}$ ) from the semiconductor into the insulator and the insulator PC (threshold $E_{g}$ (ins)). Thanks to the externally applied electric field, the thresholds $\Phi_{e}$ and $\Phi_{h}$ correspond to the energy position of the insulator band edges at the interface, not influenced by the charges distributed over the remaining thickness of the insulating layer. $E_{g}(\mathrm{sc})$ refers to the width of semiconductor bandgap.

energy shift leading to a systematic error in the measured band offset value. To account for this radiation-induced measurement artifact, a correction based on the surface potential monitoring, for example, by using the shift of the C1s core level of adsorbed carbon atoms, can be introduced. Alternatively, shifts of the core levels in substrate and in the insulating overlayer can also be used to evaluate the effect of the insulator charging $[20,28]$. Typically, the correction for charging effect in the range of $0.3-0.5 \mathrm{eV}$ is reported, see, for example, Table 2 in [20], though the charging-induced potential drop values approaching $1 \mathrm{eV}$ have been reported for some insulating materials [29]. However, accuracy of these correction procedures may further be questioned since the charging effect is sensitive to the in-depth trapped charge profile which, in general, remains unknown.

Particularly large error is expected to arise in the case of photoelectron spectroscopy analysis of stacked insulators because of additional differential charging between two stacked components which cannot be corrected by using only the value of electrostatic potential of the sample surface. This effect can be seen in the recently published results of band alignment analysis at interfaces of insulating oxides using the Kraut's method [30]. For example, the inferred CB offset between $\mathrm{Al}_{2} \mathrm{O}_{3}$ and $\mathrm{HfO}_{2}$ of $2.55 \mathrm{eV}$ is more than $2 \mathrm{eV}$ larger than the barrier differences found at interfaces of these oxides with a variety of semiconductors such as $\mathrm{Si}, \mathrm{Ge}$, and $\mathrm{GaAs}$. Obviously, this result is related to the artifact caused by the $\mathrm{X}$-ray induced insulator charging rather than reflects the real $\mathrm{CB}$ offset.

In order to avoid this ambiguity, the interface band offsets were determined using a combination of electron/hole IPE and PC spectroscopies on metal-insulator-semiconductor
(MIS) capacitors obtained by deposition of a semitransparent metal electrode on top of the oxide $[21,22,31]$. The physics of the IPE/PC technique is illustrated by the interface band diagram shown in Figure 2. When the sample is illuminated with photons of energy $(h \nu)$ exceeding the energy barrier for electrons $\left(\Phi_{e}\right)$ under positive gate bias (panel (a)) or the barrier for holes $\left(\Phi_{h}\right)$ under negative gate bias (panel (b)), charge carriers may be injected into the oxide producing a photocurrent as the carriers are drifting towards the opposite metal electrode. Thanks to the electric field in the oxide induced by biasing the top metal electrode, the measured barrier height is determined by the relative energy position of the band edges in the semiconductor and in the insulator taken at a distance of the mean photoelectron escape depth in the semiconductor and at the image-barrier top position in the insulator, respectively. With both values in the range of a few nanometers, the impact of oxide charges on the electrostatic potential variation across the interface becomes negligible. The IPE spectra are usually measured at several different strengths of the externally applied electric field. Then the spectral thresholds are found by extrapolating the quantum yield of IPE to zero at every field strength value. In turn, the extrapolation of the inferred IPE thresholds to zerofield strength eventually yields the barrier height unaffected by built-in charges.

It is also worth mentioning that IPE experiments usually require photons of much lower energy (typically below $5 \mathrm{eV}$ ) than the photons used in the photoelectron spectroscopy measurements. This prevents generation of electron-hole pairs in the insulator, thus minimizing the charging-related phenomena. In addition, by simply extending the spectral range to higher photon energies, one can also determine 
the oxide bandgap $E_{g}$ (ins) from the spectral threshold of the intrinsic PC. This oxide gap value can be used to calculate both the CB and VB offset at the interface if only one IPE barrier is measured experimentally, or, else, to provide the internal consistency proof by comparing the experimental $E_{g}$ (ins) value to the gap width calculated from the electron and hole IPE barriers $E_{g}$ (ins) $=\Phi_{e}+\Phi_{h}-E_{g}$ (sc) (cf. Figure 2).

Determination of the insulator bandgap width using PC measurements is of particular value when considering nanometer-thin insulating layers on semiconductor or metal substrate. The PC signal is proportional to the number of absorbed photons and can be considered as close analog of optical absorption measurements. Determination of optical absorption in thin layers by other optical methods, such as spectroscopic ellipsometry, is strongly influenced by the choice of the absorption model used to simulate the spectra resulting in significant uncertainty in the inferred onset of the intrinsic optical absorption. It is also possible to evaluate the insulator bandgap value from the electron energy loss spectra [32-36]; however, the need to subtract the zero-loss peak in order to recover the single-electron loss function faces a number of problems which impair accuracy of this technique. Worth adding here is that the frequently applied linear fit of the loss function is inconsistent with physics of electron energy loss through excitation of direct or indirect transitions in a solid [33] and cannot be considered as a valid procedure of threshold determination. Furthermore, if the electron energy loss spectrum associated with O1s (or N1s) photoemission peak is analyzed [37], the additional broadening of the zero-loss peak caused by Coulomb interaction of a photoelectron with a core hole must be taken into account when extracting the single-electron loss function. Since this kind of analysis is almost never done, the extracted insulator bandgap values cannot be considered as being reliable. In these circumstances, bandgap determination using PC spectroscopy offers a highly attractive alternative $[38,39]$.

\section{Basics of Internal Photoemission Spectroscopy}

Though the physical background of the IPE spectroscopy has recently been extensively overviewed [22], it is still worth of providing a reader with a short summary of its most essential features. In general the IPE process can be defined as optically stimulated transition of a charge carrier from one condensed phase into another. The transition across the interface region between two solids makes the IPE physically different from the internal photoeffect, which refers to optical generation of mobile charge carriers in a condensed phase. The internal photoeffect may occur in a variety of ways which include not only IPE but, also, excitation of PC in a homogeneous sample. The transition of the optically excited charge carrier across the interface barrier into another solid represents a key element of IPE which makes it different from the internal photoeffect. To observe the IPE, the corresponding electron transitions must provide a substantial contribution to the total flux of charge carriers across the interface. This regime is normally attained if a potential barrier of sufficient height is present at the interface. The barrier will suppress electron transitions between nonexcited (ground) states but the optically excited carriers may be transported across it. This picture allows the description of IPE by using the welldeveloped model of electron photoemission into vacuum (the external photoemission effect) [22, 31], even though the replacement of vacuum by another solid ensues nonnegligible effects, some of which will be discussed later.

The most successful descriptions of both external and internal electron photoemission processes are based on phenomenological model [40-42] considering this quantum phenomenon as a sequence of three relatively independent stages: (1) photoexcitation of a carrier inside a solid, (2) transport of the photoexcited carriers towards the emitting surface (or interface), and (3) escape of the carrier from the surface by surmounting the potential barrier. The well appreciated advantage of this model consists in providing a simple analytical scheme, which can be directly applied to the analysis of experimental results.

Further simplification can be reached by limiting the energies of photons and the excited charge carriers to the range in close vicinity of the spectral threshold of photoemission $(\Phi)$. Then one may assume that no variation in the optical properties of the studied system occurs when changing the photon energy in this narrow range [43]. The energy distribution of the excited carriers reaching the surface of the emitter will represent a (an attenuated) replica of the internal photoexcited carrier distribution $N^{*}(E)$ determined exclusively by the optical and scattering properties of the emitter material because any inelastic scattering event will make carrier escape impossible [43]. The escape probability $P$ of the carriers from the $N^{*}(E)$ distribution can in the simplest way be described by using the well known Fowler condition [44]

$$
P\left(p_{n}\right)= \begin{cases}1, & \text { if } p_{n}>p_{c} \\ 0, & \text { if } p_{n}<p_{c}\end{cases}
$$

where $p_{n}$ represents the component of the carrier momentum oriented along the normal to the emitter surface and $p_{c}$ is the "critical" escape momentum value corresponding to the particular surface/interface barrier height $\Phi=p_{c}^{2} / 2 \mathrm{~m}^{*}$, where $m^{*}$ is the effective mass of an electron. If tunnelling transitions are neglected, which, as will be shown further is the case relevant to IPE, the Fowler condition can be rewritten in the energy terms as [43]

$$
P\left(E_{n}\right)= \begin{cases}1, & \text { if } E_{n}>\Phi, \\ 0, & \text { if } E_{n}<\Phi,\end{cases}
$$

where $E_{n}$ is the kinetic energy associated with the normal component of electron momentum $p_{n}$. The transition to the total carrier energy $E$ yields $P(E)=D(E-\Phi)^{a}$ for $E>\Phi$, where $D$ is a constant and $a$ is determined by the carrier distribution in the momentum space, usually lying in the range $0.5-1[43,45]$.

The basic assumption made in deriving the above expression for $P(E)$ via (2) consists in relaxation of the momentum conservation requirement. Several arguments in favour of 
this simplifying approach have been suggested [43] including breakdown of translational symmetry at the interface, multiple bands contributing to the density of states (DOS) in the near-threshold energy range, and fast relaxation of the excited state due to scattering of electrons as evidenced by the small photoelectron escape depth $\left(\lambda_{\mathrm{sc}} \approx 1 \mathrm{~nm}\right.$ in Si near the threshold of external photoemission, see, e.g., [46]). To these arguments one may add that, as concerns the IPE into high $-\kappa$ oxide insulators, the final state of the injected carrier belongs to an amorphous or to a polycrystalline solid and, therefore, the momentum conservation requirement is lifted entirely. In this case, the number of photoemitted electrons can be calculated simply by integrating $P(E)$ and $N *(E)$ over the electron energy range exceeding the barrier height at the interface [38].

The IPE quantum yield $Y$, that is, the number of emitted electrons normalized to the number of exciting photons, can be expressed as a power function of the excess photon energy above the threshold energy $\Phi$ :

$$
Y(h v)=A(h v-\Phi)^{p},
$$

in the range $h v>\Phi$, where the exponent $p$ depends on the functional form of the excited carrier energy distribution at the surface of the emitter [43]. The spectral threshold of photoemission $\Phi$ corresponds to the minimal photon energy sufficient for electron emission and can be directly associated with the height of the energy barrier at the interface. The IPE of electrons from states close to the Fermi level of a metal (described by a step function $N^{*}(E)$ ) is predicted to follow the Fowler law $Y \sim(h v-\Phi)^{2}$ [44]. The IPE of electrons excited out of the semiconductor VB follows the $Y \sim(h \nu-\Phi)^{3}$ law [43], because $N^{*}(E)$ can be well approximated by a linear function [47]. In order to determine the spectral threshold $\Phi$ of photoemission the yield spectral curves are linearized in $Y^{1 / p}-h \nu$ coordinates and then linearly extrapolated to zero yield value.

The $N^{*}(E)$ distributions encountered in real solids and the energy-dependent scattering mechanisms [22] may cause deviation of the IPE yield spectra from the idealized cases analyzed by Powell [43] forcing one to use the exponent $p$ as a fitting parameter. In any case the photon energy range of fitting is rather limited and rarely exceeds $1 \mathrm{eV}$. For example, the energy distribution of electrons photoemitted from Si into vacuum (which should be proportional to $N^{*}(E)$ ) can be approximated by a linear function of energy below the top of silicon $\mathrm{VB}$ only within $\approx 0.8 \mathrm{eV}$ wide energy range [47]. Applying the yield fitting over a wider energy interval would definitely lead to an error in the determination of the spectral threshold.

It should also be added here that under momentum conservation restrictions, the power function yield dependence on the photon energy can still be used but with a somewhat higher $p$ value (usually by an amount of 0.5 ) than the classical Powell theory would give [48]. Perhaps, the most convincing argument in favour of the applicability of the simplified Powell model (i.e., neglecting the momentum conservation requirement) is provided by the electron IPE in structures with amorphous insulators and in samples with the same crystalline insulators grown epitaxially on the same silicon photoemitter [49]. The same spectral threshold values are found using the same spectral fits for both singlecrystal and amorphous oxides $\left(\mathrm{LaLuO}_{3}, \mathrm{Lu}_{2} \mathrm{O}_{3}\right)$ suggesting no significant influence of the momentum conservation condition per se. Therefore, extraction of the IPE threshold from extrapolation of $Y^{1 / p}-h v$ plots represents a valid procedure of the interface barrier determination, also applicable in the case of epitaxially-grown insulating layers.

Next, in the IPE experiment the thin insulating film is usually sandwiched between semiconductor and metal electrodes which enables application of an electric field of high strength $F$ (up to $10^{7} \mathrm{~V} / \mathrm{cm}$ ) by biasing the MIS capacitor. Application of the electric field affects the barrier shape and height which can be described using the potential of imagelike interaction between the photoinjected charge $q$ moving in insulator at a distance $x$ from the polarized surface of the emitter as

$$
V(x)=-\frac{q}{4 \pi \varepsilon_{0} \varepsilon_{i}(2 x)},
$$

where $\varepsilon_{0}$ is the vacuum permittivity and $\varepsilon_{i}$ is the effective image force dielectric constant. The latter is usually taken close to the relative optical permittivity of the insulator in the frequency range corresponding to the inverse transit time of electron transport across the barrier region (close to the optical frequencies, i.e., $\varepsilon_{i} \approx n^{2}$, where $n$ is the refractive index of the insulating material) $[22,31]$. In the framework of the image force model, both the barrier height $\Phi$ and the position of the barrier maximum above the emitter surface plane $x_{m}$ are expected to be field-dependent [31, 43], given as

$$
\begin{gathered}
\Phi(F)=\Phi_{0}-q\left(\frac{q F}{4 \pi \varepsilon_{0} \varepsilon_{i}}\right)^{1 / 2}, \\
x_{m}=\left(\frac{q}{16 \pi \varepsilon_{0} \varepsilon_{i} F}\right)^{1 / 2},
\end{gathered}
$$

where $\Phi_{0}$ is the zero-field barrier height (in some cases referred to as the band offset).

This kind of image-force barrier lowering (frequently referred to as the Schottky effect) makes the spectral threshold field dependent. As a result, the true zero-field barrier value $\Phi_{0}$ can only be obtained by extrapolating $\Phi(F)$ to $F=0$ by using a linear fit in the $\Phi-\sqrt{ } F$ coordinates (the Schottky plot). In the case of the conventional $\mathrm{Si} / \mathrm{SiO}_{2}$ system, the Schottky barrier lowering may be considerable, sometimes exceeding $0.5 \mathrm{eV}[31,50]$. With increasing the optical dielectric constant (from $\varepsilon_{i}=2.1$ in $\mathrm{SiO}_{2}$ to $\varepsilon_{i}=5$ in $\mathrm{ZrO}_{2},[51,52]$ ), the barrier lowering becomes smaller and for the electric field strength below $1 \mathrm{MV} / \mathrm{cm}$ it appears to be less than $0.1 \mathrm{eV}$ in most of the high $-\kappa$ insulators of interest.

It is worth adding here that there are two additional factors which must be taken into account when evaluating the strength of electric field at the injecting interface. First, in a capacitor structure most frequently used in the IPE experiments, an additional electric field may arise due to different work functions of the electrodes or due to the presence of charges in the insulating layer. In this case the zero-field 
voltage point can be found as the minimal attractive voltage at which the IPE current becomes detectable. Second, in the case of a stacked insulator, the strength of the electric field must be calculated using thicknesses and dielectric permittivities of separate layers. These properties must be found using independent experiments, for example, the transmission electron microscopy and capacitance measurements [51]. If this additional characterization is not possible, one can use the average field across the insulating stack to extrapolate the Schottky plot to zero field, since the average field is always proportional to the strength of the field in each component of the stack.

Since the major goal of this work is to quantify the interface barriers and band offsets, the influence of interface charges and electron scattering will not be addressed here. The reader can find extensive analysis of these factors in the literature $[21,22,31]$. Rather, it is worth briefly describing how the IPE experiments are carried out in practice. There are two essential requirements to extract meaningful information from the IPE experiment. First, the optical excitation of charge carriers must be realized under the condition of a static and uniform electric field applied to the interface. Second, the escape of charge carriers over the barrier into the insulator should be detected with sufficient sensitivity. The first condition leads to the simplest configuration, that is, a planar MIS capacitor with one of the electrodes transparent enough to allow the optical excitation of charge carriers at interfaces of the sandwiched insulator layer. The (semi)transparent electrode can be applied in a variety of ways such as deposition of semitransparent metal or semiconductor electrodes (10-30 nm in thickness) or, else, application of an optically transparent conducting electrolyte contact [45]. Evaporation of chemically inert Au electrodes of $13-15 \mathrm{~nm}$ thickness through a shadow mask probably provides the simplest and most feasible solution.

Detection of charge carriers overcoming the interface barrier can be done in several ways. First, one can simply measure the dc photocurrent generated when the injected carriers drift from the emitter to the opposite metal electrode. This approach has a sensitivity limited by the instability level of the dark current flowing through the insulating film at a given bias. The sensitivity of a standard electrometer $(\sim 1 \mathrm{fA})$ usually lies far below. As a remedy, one might consider the use of ac detection by applying a chopped light source. However, a large specific capacitance of samples with high- $\kappa$ insulator layers of only a few nanometers in thickness would necessitate the usage of capacitors of sufficiently small area. This, in turn, makes the design of the focusing optics quite demanding, because of the large size of the emitting body of the ark discharge lamps (used to attain a sufficient light intensity in the photon energy range $h v>3 \mathrm{eV}$ ) and the unconditional need to avoid chromatic aberrations over a broad spectral range.

All the measurements discussed in this work were performed in the dc current mode in which the IPE current was determined as the difference between the photocurrent measured under illumination and the one measured in darkness, using a Keithley 6517 or 617 electrometer. The optical scheme has been discusssed elsewhere [21, 22] in which the absolute calibration of the light intensity at the exit was attained using NIST-traceable Si $(\lambda>300 \mathrm{~nm})$ and $\mathrm{SiC}(\lambda<400 \mathrm{~nm})$ photodiodes. The spectral resolution was fixed at $2 \mathrm{~nm}$ which corresponds to a monochromator slit energy width of about $15 \mathrm{meV}$ at $h v=3 \mathrm{eV}$. A second optical beam provided was used to suppress the surface photovoltage signal arising from a semiconductor surface space charge layer generated by the short-term instability of the primary light source. The relative quantum yield $Y$ was defined in terms of the photocurrent normalized to the incident photon flux.

The application of current measurements in a MIS capacitor to detect IPE transitions generally brings about an additional problem, namely regarding the identification of the dominant photocurrent generation process [21,22]. First, since both electrodes of the MIS capacitor are illuminated, charge carriers of opposite signs may be injected from the opposite electrodes, that is, illumination of a positively biased MIS structure may lead to simultaneous IPE of electrons from the semiconductor and of holes from the metal. In the case of negative gate bias, electrons are photoemitted from the metal electrode, while IPE of holes is possible from the semiconductor. Both electrons and holes contribute equally to the photocurrent, which, in principle, makes it impossible to immediately identify the injecting interface. Though somewhat laborous, the solution to this problem lies in the comparison of the IPE characteristics of various otherwise identical MIS capacitors except for the application of different metal electrodes with strongly different work functions $[21,22]$. Should the IPE be related to the electron states of the metal, the change of the Fermi energy would lead to a corresponding shift of the IPE spectral threshold. If no corresponding shift is observed in the yield spectra, the dominant contribution to the photocurrent likely comes from the opposite electrode. For reasons of completeness, we also add that identification of the injecting interface can also make use of the optical characteristics of the electrodes, for example, the transitions between high-symmetry points in the Brillouin zone of the emitter [21, 22, 31].

The discussed two approaches in the identification of IPE-related photocurrent generation mechanisms are of particular importance in the case of insulators containing a considerable density of traps [53,54]. Charge carriers captured by these imperfections may then be optically excited in the course of IPE experiments and give rise to additional photocurrent unrelated to the IPE. The occupancy of these traps will be determined by electronic exchange with electrodes of MIS capacitors and to some extent will be sensitive to the energy position of the Fermi level in semiconductor or metal. However, the above described IPE model does not provide an adequate description of the trap photoionization and, therefore, cannot be used to extract meaningful barrier height values from the trap-assisted photocurrent spectra. In some cases, when traps are continuousluy distributed in energy inside the insulator bandgap, one may associate the upper edge of the traps occupied by electrons with the Fermi level in the nearby electrode and use extrapolation of the corresponding threshold to zero field in order to estimate the corresponding barrier [53, 54]. However, neither Powell's 
description of the yield spectra nor the image force barrier model provide physical background for such extrapolation. Rather, the best fit of the photoionization spectral curve with the exponent $p$ kept as fitting parameter can be done using, for instance, the algorithm proposed by Lange et al. $[55,56]$.

As a final point here, it is worth addressing the experimental inaccuracy involved with the IPE spectral threshold determination. There are several uncertainty sources which have been discussed in detail previously [21] ranging from the energy width of the monochromator slit to the linear extrapolation procedures used to extract the spectral threshold at zero field. Taken together as independent errors, these uncertainty contributions result in a typical experimental error in the barrier determination between \pm 0.05 and $\pm 0.10 \mathrm{eV}$. Somewhat better accuracy, close to $\pm 0.05 \mathrm{eV}$, can be achieved if measuring energy shifts of the IPE spectra which have similar shapes excludes the need for extrapolation. This kind of relative measurements can be performed, for instance, when analyzing IPE into the same insulator from different semiconductors or metals $[57,58]$.

\section{Interfaces of Semiconductors with Deposited Oxides}

In this work we will address experiments conducted on the semiconductor/insulator structures prepared by depositing an insulating oxide on the surface of a semiconductor. While amorphous insulating layers can easily be deposited by a variety of techniques, fabrication of crystalline oxides is more challenging. To obtain crystalline oxide phases we employed two approaches. First, thanks to the thermal stability of silicon, the amorphous oxide layers can be crystallized into a polycrystalline layer by using a postdeposition anneal. In some cases temperatures as high as $1000^{\circ} \mathrm{C}$ may be needed as, for instance, in the $\gamma-\mathrm{Al}_{2} \mathrm{O}_{3} / \mathrm{Si}$ system [59]. Second, if the lattice mismatch between the oxide and the substrate is not prohibitively large, the oxide layer can be grown epitaxially on (100) or (111) silicon surfaces because of the possibility to conduct this growth at a sufficiently high temperature $\left(>650^{\circ} \mathrm{C}\right)$. The crystallinity of the oxide layer was routinely assessed by X-ray Diffractometry (XRD) or Reflection High Energy Electron Diffraction (RHEED) and will not be discussed here. The annealing approach offers the advantage to enable fabrication of relatively thick $(>20 \mathrm{~nm})$ crystallized oxide layers which are usually difficult to grow epitaxially because of the limited pseudomorphic growth thickness achievable. The Si-related component of the interface band diagram remains unchanged, thus providing a reliable energy reference which allows monitoring of the oxide bandgap edge energies as a function of oxide composition and structure.

In a similar approach, semiconductor/insulator structures were also fabricated on a number of high-mobility semiconductor materials by depositing the same insulating metal oxide in order to analyze the behavior of the interface barriers as affected by the bulk DOS of the semiconductor and the crystallographic orientation of its surface plane. As the semiconductors such as $\mathrm{Ge}$ or $\mathrm{A}_{\mathrm{III}} \mathrm{B}_{\mathrm{V}}$ compounds lack the thermal stability of $\mathrm{Si}$ crystals, the amorphous $\mathrm{Al}_{2} \mathrm{O}_{3}$ layers are used as the common "frame of reference." Several other insulating oxides to be discussed, for instance, $\mathrm{HfO}_{2}$ and $\mathrm{ZrO}_{2}$, exhibit polycrystalline features if deposited at temperatures exceeding $350^{\circ} \mathrm{C}$. Nevertheless, as it will be shown below, one can obtain a meaningful comparison of the band offsets to those observed in the case of the $\mathrm{Si}$ substrate because, for cations of large ionic radius $r_{i}$, the oxide crystallinity has no measurable effect on the interface band alignment. All the MIS samples were prepared in the same way by thermoresistive evaporation of a semitransparent top electrode $(\mathrm{Au}, \mathrm{Al}, \mathrm{Ni}, \ldots)$ in vacuum. These structures were then used to acquire IPE/PC spectra as was already discussed elsewhere [21, 22].

\section{Silicon/Insulator Interfaces}

Interfaces of silicon with high-permittivity metal oxide insulators allow one to analyze the evolution of the energy barriers when varying composition and structure of the oxide overlayer while preserving silicon as a kind of reference source of electron or hole photoemission. In this section, we will show how this approach can be used to trace the influence of the oxide bandgap width on the interface barriers as affected by the oxide composition and crystallinity. Furthermore, it will be shown that neither interlayers (IL) of various compositions nor the change in crystallographic orientation of the Si photoemitter lead to any measurable interface barrier change, importantly indicating an insignificant contribution of structure-sensitive interface dipoles to the barrier.

5.1. Interfaces of Silicon with Oxides of Light Elements (Be, $\mathrm{Mg}, \mathrm{Al}, \mathrm{Si}, \mathrm{Sc}$ ). It is worth starting discussion regarding the $\mathrm{Si} /$ insulator barriers from interfaces between this semiconductor and insulating oxides of light elements, since they can directly be compared to the well studied case of $\mathrm{Si} / \mathrm{SiO}$ interface. Here we will pay particular attention to interfaces between $\mathrm{Si}$ and $\mathrm{Al}_{2} \mathrm{O}_{3}$ which represent the most interesting case for several reasons. First of all, unlike most of other metal oxides, amorphous alumina can be grown by atomic layer deposition (ALD) directly on an H-terminated Si surface (HF-dip last) resulting in an atomically abrupt interface, see, for example, transmission electron microscopy image shown in Figure 5 in $[60,61]$. This property had allowed us in the past to analyze the influence of the subsequently thermally grown $\mathrm{SiO}_{2}$ IL on the electron IPE from $\mathrm{Si}$ into $\mathrm{Al}_{2} \mathrm{O}_{3}$ and $\mathrm{Al}_{2} \mathrm{O}_{3} / \mathrm{ZrO}_{2}$ stacks $[51,53]$. Second, while the deposited $\mathrm{Al}_{2} \mathrm{O}_{3}$ is amorphous, it may be crystallized by postdeposition annealed allowing one to monitor the evolution of $\mathrm{CB}$ and $\mathrm{VB}$ offsets as the insulator bandgap width increases from 6.1-6.2 eV in amorphous alumina $\left(\mathrm{a}-\mathrm{Al}_{2} \mathrm{O}_{3}\right)$ to $8.7 \mathrm{eV}$ in polycrystalline cubic $\gamma$-alumina [59] or in the $\gamma-\mathrm{Al}_{2} \mathrm{O}_{3}$ layers epitaxially grown on the (111)Si surface [62]. Third, thanks to its ability to effectively passivate surfaces of high mobility semiconductors such as $\mathrm{Ge}$ and $\mathrm{A}_{\mathrm{III}} \mathrm{B}_{\mathrm{V}}$ compounds (at least aresenides and phosphides), $\mathrm{a}-\mathrm{Al}_{2} \mathrm{O}_{3}$ represents the best choice for the "common oxide reference" which we will later use to evaluate the relative band offsets between different semiconductors. Finally, by comparing $\mathrm{Si} / \mathrm{a}-\mathrm{Al}_{2} \mathrm{O}_{3}$ samples 


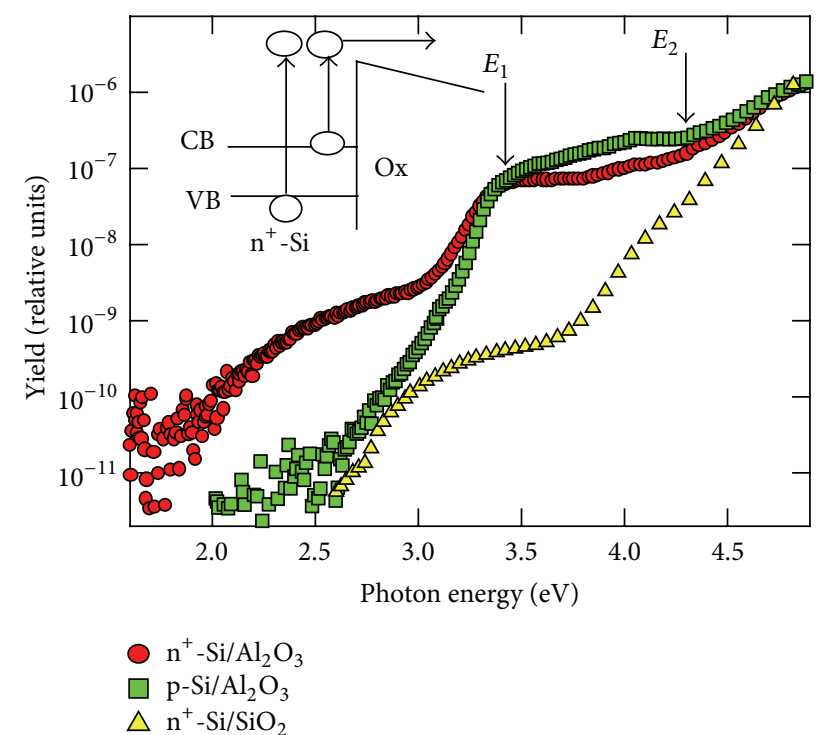

FIGURE 3: Semilogarithmic plots of the photocurrent yield as measured on $\mathrm{p}-\mathrm{Si}(100) / \mathrm{Al}_{2} \mathrm{O}_{3} / \mathrm{Au}(\square)$ and $\mathrm{n}^{+}-\mathrm{Si}(100) / \mathrm{Al}_{2} \mathrm{O}_{3} / \mathrm{Au}(\mathrm{O})$ capacitors biased to an average strength of electric field in the oxide of $2 \mathrm{MV} / \mathrm{cm}$. The insulating layer was fabricated by ALD of alumina at $300^{\circ} \mathrm{C}$. For comparison, also shown is the yield spectrum measured on a $\mathrm{n}^{+}-\mathrm{Si}(100) / \mathrm{SiO}_{2} / \mathrm{Au}$ sample for the oxide field strength of $1.57 \mathrm{MV} / \mathrm{cm}(\triangle)$. The inset schematically illustrates the observed electron IPE from the CB and VB of silicon.

prepared by different techniques such as ALD, chemical vapor deposition, molecular beam deposition (MBD), ebeam evaporation in a wide range of processing conditions, for example, when changing the substrate temperature from $-50^{\circ} \mathrm{C}$ to $420^{\circ} \mathrm{C}$, we found the same oxide bandgap and the band alignment with respect to the Si substrate. The stability of the oxide properties allows us to directly compare semiconductor/alumina interfaces prepared by using different deposition techniques at different fabrication facilities.

Let us first compare (100) $\mathrm{Si} / \mathrm{SiO}_{2}$ and (100) $\mathrm{Si} / \mathrm{Al}_{2} \mathrm{O}_{3}$ interfaces for the case of $\mathrm{a}-\mathrm{Al}_{2} \mathrm{O}_{3}$ which comes closest to the amorphous thermally grown $\mathrm{SiO}_{2}$ insulator. In Figure 3, the spectra of the photocurrent quantum yield from the low Bdoped $\left(n_{a} \approx 10^{15} \mathrm{~cm}^{-3}\right) \mathrm{p}$-Si(100) (口) and from the heavily P-doped $\left(n_{d}>10^{20} \mathrm{~cm}^{-3}\right) \mathrm{n}^{+}-\mathrm{Si}(100)(\mathrm{O})$ into the same type of a- $\mathrm{Al}_{2} \mathrm{O}_{3}$ prepared by ALD on the IMEC-cleaned [63] silicon substrates are shown. These spectra reveal that the doping-induced increase of electron density in the $\mathrm{Si} \mathrm{CB}$ results in the appearance of the additional photocurrent in the spectral range $2 \leq h v \leq 3 \mathrm{eV}$. This signal, therefore, can be associated with IPE of electrons from the silicon CB into the oxide. The second threshold at approximately $1 \mathrm{eV}$ higher energy can then be assigned to the electron IPE from the silicon VB. This assignment is independently supported by observation of kinks in the IPE yield curves at 3.3-3.4 eV and $4.3 \mathrm{eV}$ indicated by arrows $E_{1}$ and $E_{2}$ and corresponding to optical singularities of the Si crystal. The spectrum of electron IPE from the similarly heavily-doped $\mathrm{n}-\mathrm{Si}$ into $\mathrm{SiO}_{2}$ $(\triangle)$ exhibits the same structure as the $\mathrm{n}^{+}-\mathrm{Si} / \mathrm{Al}_{2} \mathrm{O}_{3}$ sample but shifted by $\approx 1 \mathrm{eV}$ toward higher photon energies. The latter

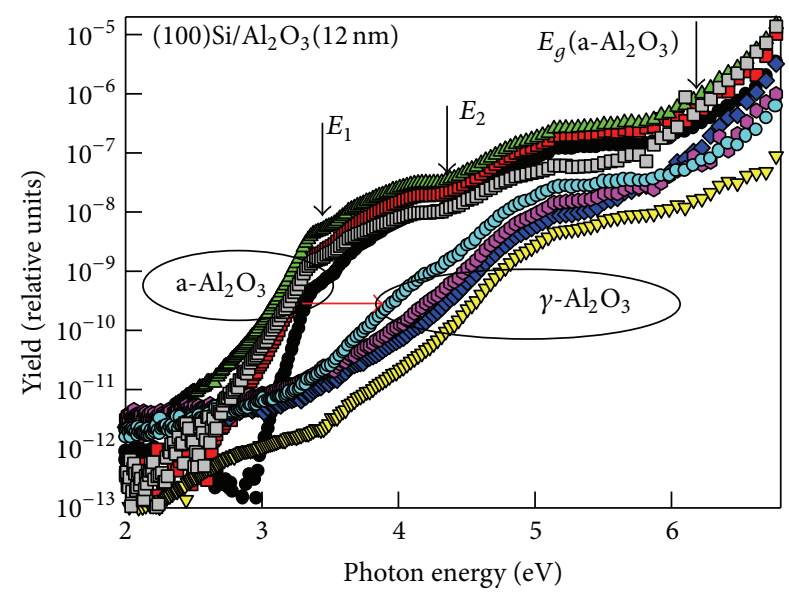

$$
\begin{array}{ll}
V \text { (Volt) } & \\
\bullet+1 \mathrm{~V} & \nabla+2 \mathrm{~V} \\
\square+2 \mathrm{~V} & \diamond+3 \mathrm{~V} \\
\Delta+3 \mathrm{~V} & 0+4 \mathrm{~V} \\
& 0+5 \mathrm{~V}
\end{array}
$$

FIGURE 4: Semilogarithmic plot of the quantum yield of electron IPE from $\mathrm{Si}$ as a function of photon energy measured on $\mathrm{Si} / \mathrm{Al}_{2} \mathrm{O}_{3} / \mathrm{Au}$ samples with an as-deposited $12 \mathrm{~nm}$ thick amorphous (a) ALD $\mathrm{Al}_{2} \mathrm{O}_{3}$ layer, as compared to the polycrystalline ( $\gamma$-phase) $\mathrm{Al}_{2} \mathrm{O}$ (same samples) obtained by annealing at $1000^{\circ} \mathrm{C}$ (filled symbols). Vertical arrows indicate energies of direct optical transitions in the $\mathrm{Si}$ substrate crystal acting as the source of photoelectrons as well as the onset of intrinsic photoconductivity (the bandgap, $E_{g}$ ) of a- $\mathrm{Al}_{2} \mathrm{O}_{3}$.

reflects a $1 \mathrm{eV}$ difference in the $\mathrm{Si}$ /oxide electron barrier height corresponding to $1 \mathrm{eV}$ difference in electron affinity between $\mathrm{SiO}_{2}$ and a- $\mathrm{Al}_{2} \mathrm{O}_{3}$. This example shows how variations in the oxide $\mathrm{CB}$ position are directly reflected in the electron IPE spectra.

To further illustrate the use of the IPE spectroscopy to monitor the energy position of the oxide CB bottom edge, we address the impact of crystallization on the band alignment between silicon and $\mathrm{Al}_{2} \mathrm{O}_{3}$. While amorphous a- $\mathrm{Al}_{2} \mathrm{O}_{3}$ films exhibit only a 6.1-6.2 eV wide gap [52], crystallization into cubic $\gamma-\mathrm{Al}_{2} \mathrm{O}_{3}$ by $1000^{\circ} \mathrm{C}$ annealing eliminates the low-energy PC [59] reflecting an increase in the gap width to $8.7 \mathrm{eV}$ value which was reported previously for this $\mathrm{Al}_{2} \mathrm{O}_{3}$ phase [64]. The most interesting aspect here is the evolution of the $\mathrm{VB}$ and $\mathrm{CB}$ edges caused by the transition from the amorphous to the cubic $\mathrm{Al}_{2} \mathrm{O}_{3}$. Electron IPE spectra, such as shown in Figure 4 for the as-deposited a- $\mathrm{Al}_{2} \mathrm{O}_{3}$ and polycrystalline $\gamma$ $\mathrm{Al}_{2} \mathrm{O}_{3}$, indicate a $\sim 0.4 \mathrm{eV}$ upshift of the oxide $\mathrm{CB}$ relative to the reference level of the Si VB top. This shift is also consistent with the results of electron IPE measurements performed on single-crystal $\gamma$ - $\mathrm{Al}_{2} \mathrm{O}_{3}$ films epitaxially grown on (111) Si at $775^{\circ} \mathrm{C}$ [62]. Therefore, one may conclude that the crystallization-induced gap widening in $\mathrm{Al}_{2} \mathrm{O}_{3}$ from 6.2 to $8.7 \mathrm{eV}$ occurs for about $80 \%$ through a downshift of the $\mathrm{Al}_{2} \mathrm{O}_{3}$ VB top.

In order to determine the interface barrier height one first needs to extract the spectral thresholds of IPE from the quantum yield spectra and then to analyze their field 


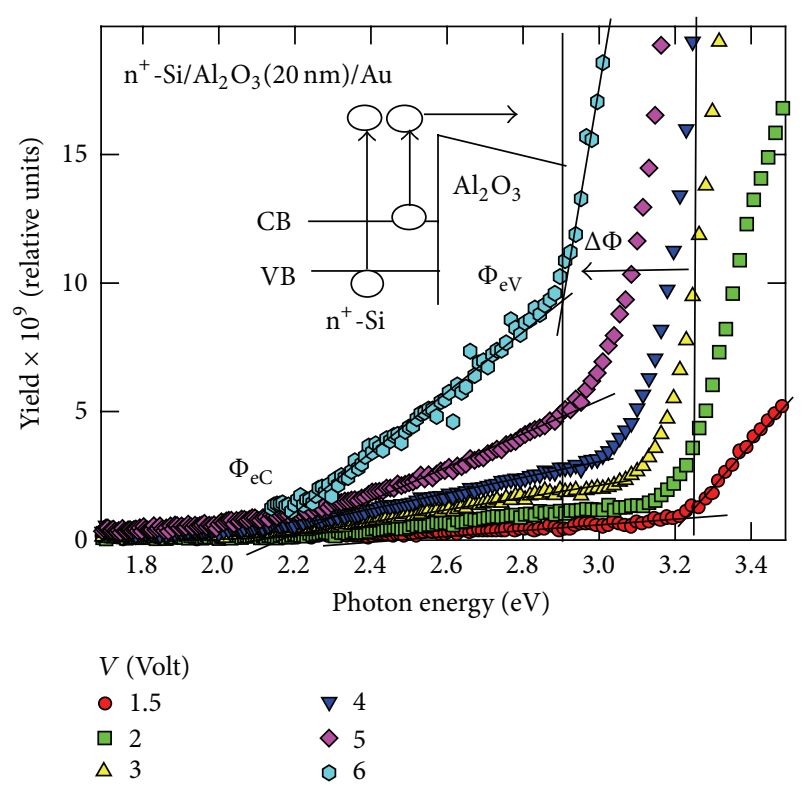

FIGURE 5: Linear plots of the photocurrent quantum yield in a $\mathrm{n}^{+}-\mathrm{Si}(100) / \mathrm{Al}_{2} \mathrm{O}_{3}(20 \mathrm{~nm}) / \mathrm{Au}$ capacitor measured under different positive voltages $V$ applied to the metal electrode. Arrow $\Delta \Phi_{e}$ indicates the observed field-induced spectral threshold shift. The inset schematically illustrates the observed electron IPE from the CB and VB of silicon corresponding to the spectral thresholds $\Phi_{\mathrm{eC}}$ and $\Phi_{\mathrm{eV}}$, respectively.

dependence. In the analytically most simple case of electron IPE from the CB of the heavily-doped n-type Si the linear yield plots versus photon energy can directly be used to find the spectral thresholds, as is illustrated in Figure 5. These data additionally reveal two important features. First, the quantum yield of electron IPE from a narrow energy distribution close to the bottom of the silicon $\mathrm{CB}$ perfectly follows the predicted linear behavior. This observation indicates that the transitions associated with under-barrier electron tunneling do not provide any measurable contribution to the observed photoemission even in the presence of a $0.7-0.8 \mathrm{~nm}$ thick $\mathrm{Si}$ oxide IL expected to be present at the interface after IMEC cleaning [63] which includes sample treatment in ozonated water as the final step. Second, the onset of electron IPE from the silicon $\mathrm{CB}$ and $\mathrm{VB}$ is seen to be field-dependent reflecting the above discussed image-force barrier lowering. This observation indicates that the recently made suggestion regarding the optical nature of the photocurrent increase at 3.3-3.4 eV at interfaces of silicon with oxide insulators finds no experimental support [65] and reveals this threshold to be the true IPE onset. More details concerning the interpretation of the near-threshold features in the IPE spectra from silicon, including artifacts associated with optical excitation of trapassisted transitions, can be found in the recently published comment [54].

Illustrating the final step in the band alignment determination, Figure 6 shows the Schottky plot of the electron IPE spectral thresholds corresponding to photoemission of

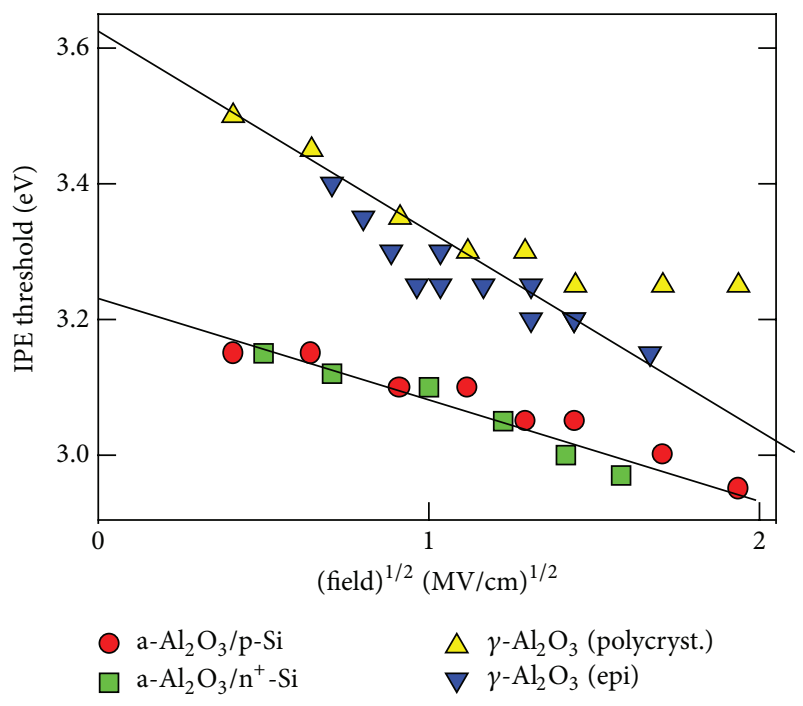

FIGURE 6: Schottky plot of the field-dependent electron IPE thresholds as measured on samples with different types of insulating $\mathrm{Al}_{2} \mathrm{O}_{3}$ grown on silicon crystal substrate. Lines illustrate linear extrapolation used to infer the zero-field energy barrier between the top of the $\mathrm{Si} \mathrm{VB}$ and the bottom of the $\mathrm{Al}_{2} \mathrm{O}_{3} \mathrm{CB}$.

electrons from the Si VB at the interfaces with a- and $\gamma$ $\mathrm{Al}_{2} \mathrm{O}_{3}$ insulators $\left(Y^{1 / 3}-h v\right.$ plots are not shown since these were illustrated in previous publications several times). Extrapolation to zero field yields a $0.4 \mathrm{eV}$ difference in the electron barrier height which reflects the shift in the oxide CB bottom as measured with respect to the reference level of the Si VB top edge. Noteworthy here is the importance of the low-field measurements which help to improve the accuracy of the Schottky plot extrapolation. As the final remark, one may add that the $2.1 \mathrm{eV} \mathrm{CB}$ offset between Si and a- $-\mathrm{Al}_{2} \mathrm{O}_{3}$ combined with the the $6.2 \mathrm{eV}$ gap width of the oxide correspond to the hole IPE barrier of $4.1 \mathrm{eV}$ which coincides with the recently reported value obtained in graphene-gated $\mathrm{Si} / \mathrm{a}-\mathrm{Al}_{2} \mathrm{O}_{3}$ entities [66].

Turning now to other studied oxides of light metals such as $\mathrm{MgO}$ [67] and $\mathrm{Sc}_{2} \mathrm{O}_{3}[49,68]$, the transition from the amorphous to the crystalline cubic phase has been found to occur without any measurable change in the electron IPE threshold (within the above indicated experimental accuracy of $\pm 0.1 \mathrm{eV}$ ). This leaves the oxide VB shift entirely responsible for the crystallization-induced bandgap widening from $6.1 \mathrm{eV}$ to $7.8 \mathrm{eV}$ in $\mathrm{MgO}$ and from $5.6 \mathrm{eV}$ to $6.0 \mathrm{eV}$ in $\mathrm{Sc}_{2} \mathrm{O}_{3}$. Recently published results regarding band alignment at the $\mathrm{Si} / \mathrm{BeO}$ interface [69] indicate that the CB offset slightly increases upon postdeposition anneal from $2.3 \mathrm{eV}$ to $2.6 \mathrm{eV}$, which is comparable to the above discussed case of $\mathrm{Al}_{2} \mathrm{O}_{3}$ but without measurable oxide VB edge shift. However, it needs to be mentioned that the studied $\mathrm{BeO}$ layers were already crystallized upon atomic layer deposition on Si [69-71].

Summarizing the results concerning band lineup at interfaces of silicon with insulating oxides of light elements, in Figure 7 are shown relative positions of the bandgaps of the discussed oxides referenced to the silicon VB top. 


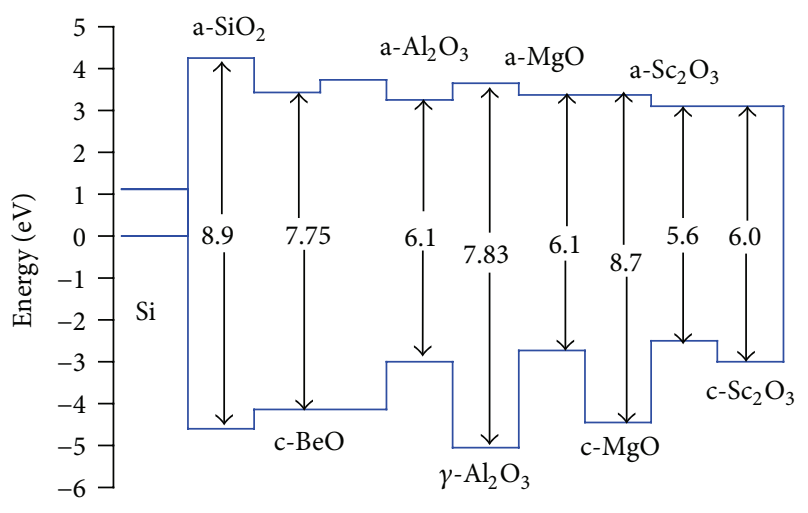

FIGURE 7: Schematic of band alignment at the interfaces of silicon with oxides of several light metals. The diagrams are shown for amorphous (a-) and crystalline cubic $\left(\gamma^{-}, \mathrm{c}^{-}\right)$phases. The origin of the energy scale is at the top of the silicon VB.

The band offsets and bandgaps are indicated according to the IPE/PC or optical data available in the literature for a$\mathrm{SiO}_{2}[72,73]$, c- $\mathrm{BeO}[69]$, a- and $\gamma-\mathrm{Al}_{2} \mathrm{O}_{3}[51,52,59,62]$, aand $\mathrm{c}-\mathrm{MgO}$ (cubic phase) $[67,74]$, and a- and c- $\mathrm{Sc}_{2} \mathrm{O}_{3}$ (cubic phase) $[49,68]$. A comparison between the bandgap edge energies of the last three oxides in amorphous and crystalline (all cubic) phases reveals an interesting trend: most of the crystallization-induced bandgap widening occurs through the shift of the VB top edge. Taking into account that the electron states near the VB top in these oxides are derived from the $2 p$ lone-pair orbitals of $O$ anions [75], crystallization to a denser phase results in lowering of the lone-pair electron energy. The latter indicates a kind of attractive interaction (e.g., the exchange one) between the O2p lone-pair electrons to become stronger. The effect appears to be the largest for the most compact cation $\left(\mathrm{Al}^{+3}\right)$ suggesting the distance between anions to be the essential factor influencing the VB top energy. Therefore, in the subsequent analysis the band offsets will be presented as functions of the (average) cation radius in the corresponding oxide [76,77].

5.2. Interfaces of Silicon with Oxides of Transition Metals ( $Y$, $\mathrm{Zr}, \mathrm{Hf}, \mathrm{Ti}, \mathrm{Ta}, \mathrm{Nb}$ ). Oxides of transition metals attract a lot of technological interest since they have by far higher dielectric permittivity than the above discussed oxides of the light elements. However, this increase in $\kappa$-value comes at the price of the lower bandgap width which is decreasing from $5.6-5.9 \mathrm{eV}$ in $\mathrm{HfO}_{2}, 5.6 \mathrm{eV}$ in $\mathrm{Y}_{2} \mathrm{O}_{3}$, and $5.4-5.5 \mathrm{eV}$ in $\mathrm{ZrO}_{2}$ to $\approx 4-4.4 \mathrm{eV}$ in amorphous $\mathrm{Nb}_{2} \mathrm{O}_{5}, \mathrm{Ta}_{2} \mathrm{O}_{5}$, and $\mathrm{TiO}_{2}$ [21]. In three latter cases the narrow bandgap results in low interface barriers preventing meaningful IPE analysis by allowing a high leakage current in MIS capacitors. Under these circumstances the band offsets were determined in complex Hf:Ti and Hf:Ta oxides and correspond to an amorphous sub-network of Ti and Ta oxides [78-80].

Another complication is related to the presence of a $\mathrm{SiO}_{2}$-like IL between $\mathrm{Si}$ and the high- $\kappa$ metal oxide which is often introduced to improve nucleation of the oxide during ALD process. This layer may potentially introduce an

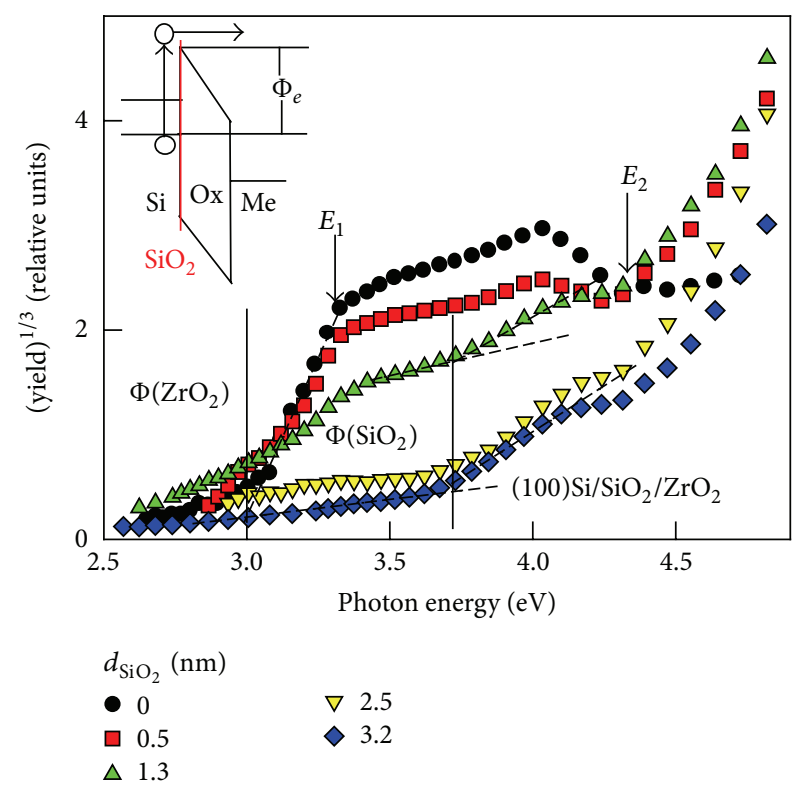

FIGURE 8: Cube root of the IPE yield as a function of photon energy for MOS capacitors with a $7.4 \mathrm{~nm}$ thick $\mathrm{ZrO}_{2}$ insulator stacked with different $\mathrm{SiO}_{2}$ ILs as illustrated in the inset. All curves are measured under an electric field of $2 \mathrm{MV} / \mathrm{cm}$ in the insulating layer closest to $\mathrm{Si}$, that is, $\mathrm{ZrO}_{2}$ in the sample with $d_{\mathrm{SiO}_{2}}=0$ or $\mathrm{SiO}_{2}$ in other samples. The arrows $E_{1}$ and $E_{2}$ indicate onsets of direct optical transition in the silicon crystal. The spectral thresholds of electron IPE from the VB of Si into different oxides are indicated by vertical lines.

additional barrier affecting the results of IPE experiments. Because of the importance of this problem, the impact of the stacked insulator structure on the IPE spectra has been addressed from the very beginning [51]. This has been done by using the controlled incorporation of $\mathrm{SiO}_{2}$ IL to the initially abrupt $\mathrm{Si} / \mathrm{Al}_{2} \mathrm{O}_{3}$ or $\mathrm{Si} / \mathrm{ZrO}_{2}$ interfaces fabricated by ALD of the corresponding oxide on the H-terminated (HF-dip last) (100)Si surface. A typical result is exemplified in Figure 8 which shows $Y^{1 / 3}-h v$ plots of the photocurrent yield in $\mathrm{Si} / \mathrm{SiO}_{2} / \mathrm{ZrO}_{2} / \mathrm{Au}$ capacitors with different thickness of the $\mathrm{SiO}_{2}$ IL. The indicated $E_{1}$ and $E_{2}$ optical singularities observed in the spectra leave little doubt that the photocurrent contains a significant contribution of electron IPE from silicon. The spectra exhibit a clear threshold close to $3 \mathrm{eV}$, similar to that observed at the $\mathrm{Si} / \mathrm{Al}_{2} \mathrm{O}_{3}$ interfaces but also show a subthreshold photocurrent which requires identification. The latter has been achieved by performing several additional experiments. First, with increasing $\mathrm{SiO}_{2}$ thickness, the photocurrent with spectral onset at $3 \mathrm{eV}$ and clear fingerprints of Si optical features is gradually eliminated while the "tail" stretching below $3 \mathrm{eV}$ remains and even increases in some samples [51]. This result suggests that these two photocurrents have different excitation mechanisms. Furthermore, the photocurrent below $3 \mathrm{eV}$ spectral threshold has been found to decay in time suggesting the electron bath of initial states to be partially exhausted. This would correspond to the defect-assisted injection rather than 

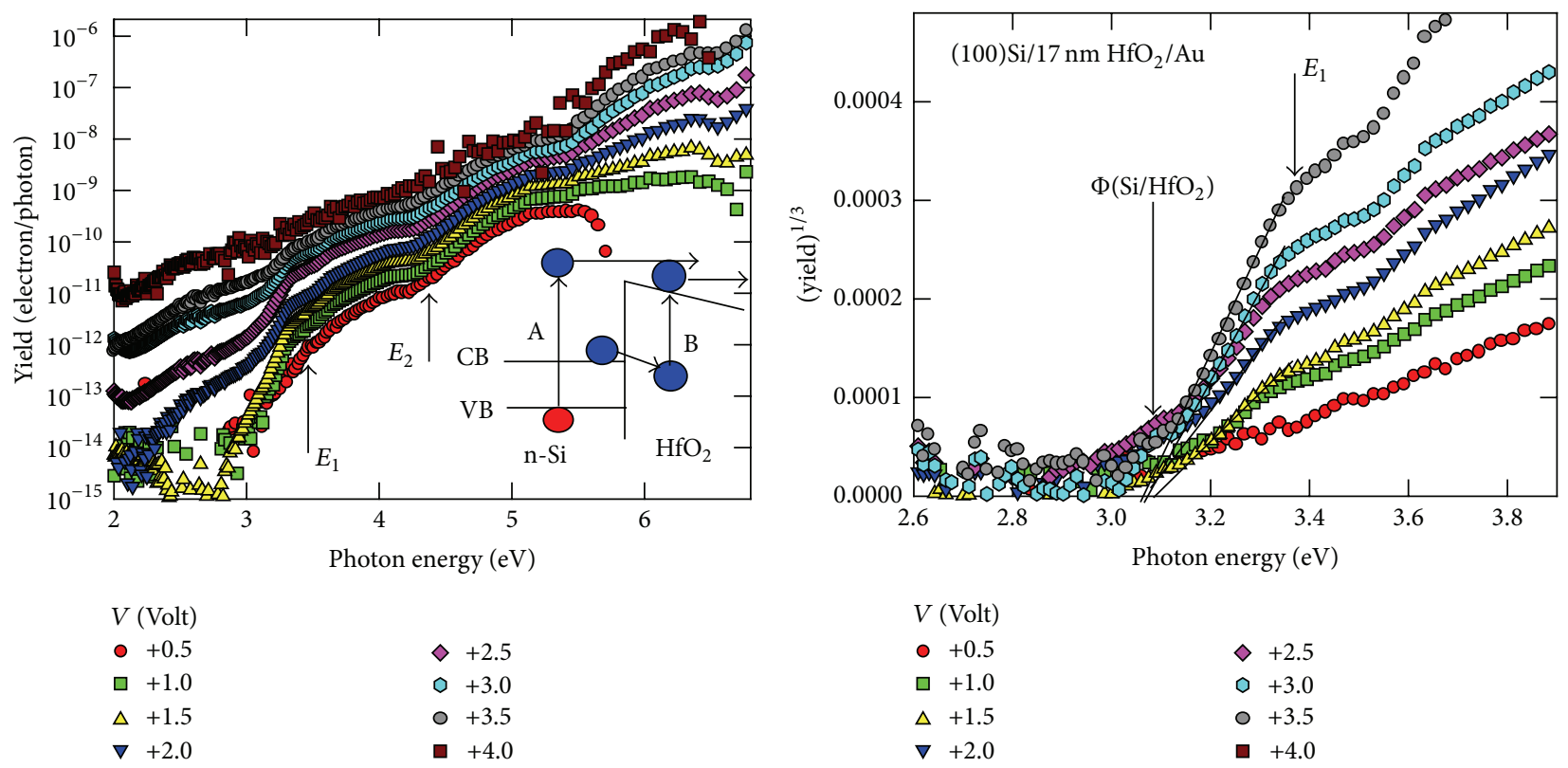

(a)

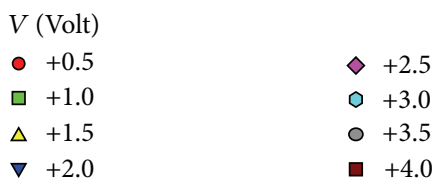

(b)

FIGURE 9: (a) Semilogarithmic plot of the IPE quantum yield as a function of photon energy measured on $\mathrm{n}$-type $\left(\mathrm{P}\right.$-doped, $n_{d} \approx(1-3) \times$ $\left.10^{15} \mathrm{~cm}^{-3}\right)(100) \mathrm{Si} / \mathrm{HfO}_{2}(17 \mathrm{~nm}) / \mathrm{Au}$ sample with the applied bias varying from $1 \mathrm{~V}$ to $4 \mathrm{~V}$. The inset illustrates the schematic of the IPE type electron transitions (A) and the transitions associated with photoionization of near-interface oxide traps ("pseudo-IPE", B). The arrows $E_{1}$ and $E_{2}$ indicate onsets of direct optical transition in the silicon crystal. (b) Cube root of the IPE yield as a function of photon energy as inferred from the data shown in the panel (a) after subtracting the "pseudo-IPE" background photocurrent. The inferred spectral threshold $\Phi$ of electron IPE from the VB of Si into the $\mathrm{CB}$ of $\mathrm{HfO}_{2}$ is indicated by the vertical arrow.

IPE from the Si crystal. Obviously then, these two photocurrent generation mechanisms are not correlated to one another.

In order to verify the hypothesis about nonIPE origin of the low-energy photocurrent, we examined in more detail the field dependence of the yield spectra. As it is exemplified in Figure 9 (a) for the case of $(100) \mathrm{Si} / \mathrm{HfO}_{2}$ interface, the photocurrent band with threshold at around $3 \mathrm{eV}$ and well pronounced Si features are visible in low fields. However, when increasing the positive voltage applied to the top gold electrode silicon contribution to the total signal gradually disappears resulting in a featureless spectrum at the highest field applied. Besides affirming different origins of the two photocurrents, this observation indicates electron tunneling to electron traps in $\mathrm{HfO}_{2}$ layer with their subsequent photoionization as the additional photocurrent generation mechanism (labeled as B in the inset in Figure 9(a)) as opposed to the true IPE process A. In order to find the spectral threshold of IPE in the presence of additional photocurrent signal, the latter has been simulated by the polynomial fit, extrapolated to the spectral region $h v>3 \mathrm{eV}$, and then subtracted from the total photocurrent. The resulting IPE spectrum has been analyzed using $Y^{1 / 3}-h v$ plots as shown in Figure 9(b), revealing a weakly field-dependent spectral threshold close to $3-3.1 \mathrm{eV}$. The weak field dependence is consistent with the high electronic component of the dielectric permittivity of $\mathrm{HfO}_{2}$ resulting in a weak image force interaction between a photoemitted electron and silicon [21].
Although it will not be discussed in depth here, experiments comparing ILs of different thickness and composition $\left(\mathrm{SiO}_{2}, \mathrm{SiON}, \mathrm{Si}_{3} \mathrm{~N}_{4}\right)$ indicate that the interface barrier for electrons in $\mathrm{Si} / \mathrm{HfO}_{2}$ entities is reproduced with the mentioned measurement accuracy of $0.05-0.1 \mathrm{eV}$ suggesting, once again, the absence of significant IL-sensitive dipole contributions to the barrier [21, 81]. Furthermore, at the interfaces of $\mathrm{HfO}_{2}$ and $\mathrm{ZrO}_{2}$ with silicon IPE of holes has also been observed [81] allowing one to reference the positions of the oxide $\mathrm{VB}$ and $\mathrm{CB}$ of the oxide with respect to the bandgap edges in silicon. The energy threshold of hole IPE from Si into $\mathrm{HfO}_{2}$ was found to be $3.6 \mathrm{eV}$ [81] which, being combined with the zero-field electron barrier of $3.15 \mathrm{eV}$, corresponds exactly to the lowest PC threshold of $5.6 \mathrm{eV}$ in the studied $\mathrm{HfO}_{2}$ layers. There has been found, however, the second threshold of $\mathrm{PC}$ at $5.9 \mathrm{eV}$ [81] which is suggestive of two phases present in the deposited hafnia. The lowest bandgap at $5.6 \mathrm{eV}$ has later been assigned to the presence of the monoclinic (m-) $\mathrm{HfO}_{2}$ phase on the basis of correlation between XRD and optical [82] or PC [83] measurements. In its turn, the optical absorption and PC threshold at $5.9 \mathrm{eV}$ has been assigned to the presence of amorphous hafnia [82, 84] which can be stabilized, for instance, by incorporating $\mathrm{Al}$ into the film which prevents formation of the $\mathrm{m}-\mathrm{HfO}_{2}[85,86]$. Interestingly, the direct comparison of electron barriers at the interfaces of $\mathrm{Si}$ with $\mathrm{m}$ - and a- $\mathrm{HfO}_{2}$ reveals no measurable difference suggesting the gap narrowing in $\mathrm{m}-\mathrm{HfO}_{2}$ to occur at the VB side of the oxide bandgap [84]. 


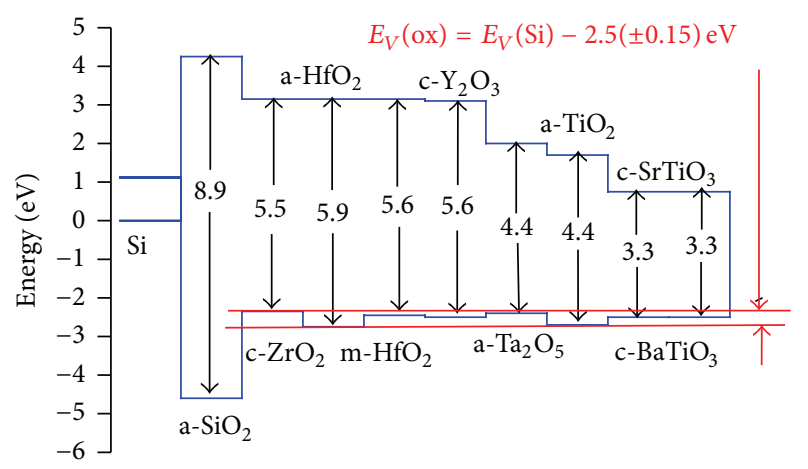

FIGURE 10: Schematic of band alignment at the interfaces of silicon with oxides of several transition and rare-earth metals as compared to the $\mathrm{Si} / \mathrm{SiO}_{2}$ interface. The diagrams are shown for amorphous (a-) and crystalline (c-) phases. The origin of the energy scale is located at the top of the silicon VB.

The available results concerning band offsets at interfaces between $\mathrm{Si}$ and several transition metal oxides are summarized in Figure 10 as compared to the $\mathrm{Si} / \mathrm{SiO}_{2}$ interface band diagram. These results reveal an important change in the band offset evolution trend as compared to the oxides of lighter elements (cf. Figure 7). The top of the $\mathrm{VB}$ remains close to $2.5 \mathrm{eV}$ within the energy interval of $\pm 0.15 \mathrm{eV}$ which may be considered as the upper limit of the structure-sensitive dipole contribution to the barrier height. The bandgap variations are mostly reflected at the $\mathrm{CB}$ side of the oxide bandgap. Furthermore, this conclusion finds independent confirmation from other barrier offset measurements. For example, the VB offset at the interfaces of (100)Si with perovskite $\mathrm{SrTiO}_{3}$ and $\mathrm{BaTiO}_{3}$ also appears to be in the indicated range of $2.5 \pm 0.15 \mathrm{eV}$ [87] and is added to Figure 10 . Note, these XPS results may be considered as sufficiently accurate since no correction for insulator charging is required because of negative $\mathrm{CB}$ offset at these interfaces, ensuring sufficient supply of electrons to the oxide. As it will be shown further, the revealed nearly-constant energy of the $\mathrm{O} 2 \mathrm{p}$ electron states at the oxide $\mathrm{VB}$ top with respect to the $\mathrm{Si} \mathrm{VB}$ edge (the so-called common anion rule) is also the case at the interfaces of silicon with the rare-earth and complex oxides.

Before continuing with analysis of behavior of interfaces of rare-earth oxides and complex oxides, it is worth briefly discussing the electronic properties of the nanometer-thin $\mathrm{SiO}_{2}$ ILs frequently encountered at interfaces of $\mathrm{Si}$ with nonnative oxides. As it can be seen from the band alignment schemes shown in Figures 7 and 10 and corresponding to sufficiently thick ( $>5 \mathrm{~nm}$ ) oxide films, the $\mathrm{SiO}_{2}$ IL might be expected to provide a $1 \mathrm{eV}$ additional barrier at the $\mathrm{CB}$ side of the bandgap and a $2 \mathrm{eV}$ barrier at the VB side. While the blocking effect of $\mathrm{SiO}_{2}$ IL is well pronounced in the case of hole IPE into the oxide VB [21, 81], it appears by far less efficient in blocking electron IPE from Si into the metal oxide CB. For instance, the spectral curves shown in Figure 8 indicate that a $1.3 \mathrm{~nm}$ thick $\mathrm{SiO}_{2}$ IL attenuates the electron IPE from Si into $\mathrm{ZrO}_{2}$ but does not block it despite the fact that the barrier of electron IPE into the CB of $\mathrm{SiO}_{2} \Phi\left(\mathrm{SiO}_{2}\right)$ is considerably higher than the barrier for the IPE into $\mathrm{ZrO}_{2} \Phi\left(\mathrm{ZrO}_{2}\right)$. This observation would suggest that $\mathrm{a} \approx 1 \mathrm{~nm}$ thick $\mathrm{SiO}_{2}$ IL constitutes no substantial barrier for electrons arriving from the Si side of the interface and acts more like a scatterer.

At first sight the last conclusion seems to contradict the well-known result of DiStefano [50] who suggested that the energy of the $\mathrm{SiO}_{2} \mathrm{CB}$ bottom is preserved down to a distance of about $0.4 \mathrm{~nm}$ from the $\mathrm{Si} / \mathrm{SiO}_{2}$ interface plane. However, more elaborated analysis points towards several factors which may lead to the smaller height of the ILrelated barrier if not completely eliminating it. First, in the case of a thin insulating layer sandwiched between two materials with a higher dielectric permittivity, the image force interaction must be considered at both interfaces of the IL [88]. This problem has been given extensive consideration several decades ago [89-91] which reveals strong additional lowering of the barrier due to polarization of the second interface. Second, while in experiments of DiStefano the $\mathrm{SiO}_{2}$ thickness was in excess of $100 \mathrm{~nm}$ [50], the band structure of the $\mathrm{SiO}_{2}$ on $\mathrm{Si}$ is expected to become thickness sensitive, while the layer becomes thinner than $1 \mathrm{~nm}$ [92-95]. The most profound effect of the $\mathrm{SiO}_{2}$ thickness reduction consists in the bandgap shrinking which is equivalent to the interface barrier lowering. The "bulk-like" CB position in $\mathrm{SiO}_{2}$ is reached at about $0.8 \mathrm{~nm}$ from the surface of silicon. Taking into account that the distance of the same order must be taken from both interfaces of the $\mathrm{SiO}_{2}$ IL, this estimate appears to be in good agreement with experimental findings [51], indicating that at least $1.3 \mathrm{~nm}$ of $\mathrm{SiO}_{2}$ IL thickness is required to start observing the $\mathrm{SiO}_{2}$ bottom CB edge (barrier $\Phi(\mathrm{Si})$ in Figure 8).

Finally, because of the extended quantum-mechanical nature of electron states in the CB of metal oxides, they will unavoidably "tail" into the gap of IL due to the tunneling effect, resulting in a projection of the high- $\kappa$ insulator CB DOS in the $\mathrm{SiO}_{2}$ IL bandgap [96]. The density of these "interface induced gap states" is expected to decrease when increasing the IL thickness which agrees with experimental observations (cf. Figure 8). However, it is this projected DOS of the high- $\kappa$ oxide CB states inside the IL that will determine the escape probability and the IPE yield. This model explains the absence in the experimental spectra of any measurable IPE threshold shift expected to be caused by voltage drop across the IL [65]. In the example shown in Figure 7 a field of $2 \mathrm{MV} / \mathrm{cm}$ strength inside the IL would result in a $0.1 \mathrm{eV}$ and $0.26 \mathrm{eV}$ shift of the $\mathrm{ZrO}_{2} \mathrm{CB}$ bottom if measured behind a 0.5 - and $1.3-\mathrm{nm}$ thick $\mathrm{SiO}_{2} \mathrm{IL}$, respectively. The obvious absence of this threshold shift in the experimental spectra indicates that the simple "stacked" $\mathrm{SiO}_{2} /$ high- $\kappa$ barrier model is irrelevant to the studied Si/high- $\kappa$ oxide interfaces. Rather, the available experimental results suggest that the presence of IL in general does not lead to a considerable variation of the high- $\kappa$ oxide band edge energies observed in the IPE experiment. More evidence in favor of this conclusion will be provided by experimental results discussed in the following sections. 
5.3. Interfaces of Silicon with Oxides of Rare-Earth Metals and Complex Oxides. Oxides of rare-earth (RE) metals and complex oxides on their base (aluminates, scandates, ...) not only provide the desirably high dielectric permittivity but also allow great flexibility in the growth conditions. As a result, they can be fabricated by a wide variety of techniques both in amorphous and crystalline phases on differently oriented faces of silicon crystals which enabled extensive IPE analysis of various aspects of the interface band alignment properties. Since many of the revealed features have already been presented in the literature and extensively overviewed $[21,22,77,97]$, here we will list the most essential findings and illustrate them by previously unpublished experimental results. However, before addressing these experimental observations, it is worthwhile to indicate the difficulties in the analysis of the electronic properties of RE-oxide-based insulators.

First of all, there is available only limited information regarding the bulk crystal bandgap width in the RE oxides. A frequently cited optical study [98] in which single-crystal gap widths for most of the RE sesquioxides are listed is actually based on a rather questionable method of the optical absorption threshold determination. The latter has been evaluated as the onset of optical transmission of $0.1 \%$ in the sample of about $0.2 \mathrm{~cm}$ thickness if coming from the short wavelength spectral range [99] rather than from the analysis of the optical absorption spectra. The accuracy of this estimate may be low as can be noticed from the estimated optical absorption edge of $4.15 \mathrm{eV}$ for the yttrium-stabilized $\mathrm{ZrO}_{2}$ crystals [100], to be compared to the bandgaps of 5.5 and $5.6 \mathrm{eV}$ for deposited polycrystalline $\mathrm{ZrO}_{2}$ and $\mathrm{Y}_{2} \mathrm{O}_{3}$, respectively. Therefore, determination of the bandgap width in the studied RE oxide layers is still necessary.

Furthermore, the PC spectra of deposited thin layers of RE-based oxides systematically exhibit "tail" stretching to $\approx 1 \mathrm{eV}$ below the onset of intense PC suggesting the presence of the band-tail states [21, 22, 49, 68, 101-105]. Indeed, amorphous $\mathrm{RE}$ oxide layers are found to exhibit a more intense subthreshold PC than their crystalline (epitaxiallygrown) counterparts which has been correlated with the corresponding "tail" in the electron IPE from Si into the oxide $\mathrm{CB}[21]$, supporting the suggestion regarding the $\mathrm{CB}$ tailing in RE oxides [101]. Nevertheless, these "tail" states are not eliminated even in the single-crystal epitaxiallygrown $\mathrm{RE}$ oxide layers. Furthermore, as it is revealed by the PC spectra measured on $\mathrm{Si} / \mathrm{Gd}_{x} \mathrm{Al}_{2-x} \mathrm{O}_{3}$ samples of different composition and shown in Figure 11, two different PC spectral thresholds appear in some cases. Noteworthy here is that these samples are prepared using ALD while no such effect is found in $\mathrm{Gd}_{2} \mathrm{O}_{3}$ layers grown by molecular beam epitaxy $[104,105]$. Apparently, phase composition of the RE oxides is significantly sensitive to the layer growth method. Obviously then, in the case of not uniquely defined bandgap width, the oxide VB top energy cannot be calculated from the measured electron IPE barrier. Fortunately enough, in many cases, the VB offset between $\mathrm{Si}$ and RE-based insulators can be measured independently by using IPE of holes from Si [101, 104, 105]. These results have allowed us to evaluate the transport gap in RE oxide insulators by

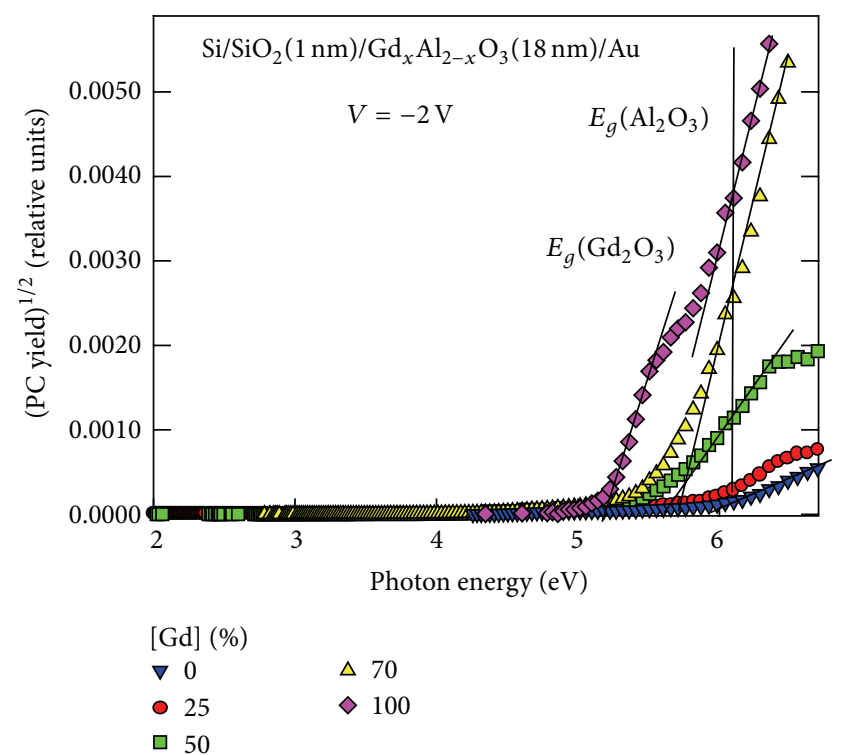

FIGURE 11: Square root of the PC yield as a function of photon energy in $\mathrm{Si} / \mathrm{SiO}_{2} / \mathrm{Gd}_{x} \mathrm{Al}_{1-x} \mathrm{O}_{3} / \mathrm{Au}$ capacitors with different concentration of gadolinium in the insulating layer.

combining the barriers inferred from the electron and hole IPE spectra rather than from the PC or optical measurements. The bandgaps are found to be in the range $5.6-5.9 \mathrm{eV}$ for a broad variety of materials addressed so far $\left(\mathrm{Gd}_{2} \mathrm{O}_{3}, \mathrm{Lu}_{2} \mathrm{O}_{3}\right.$, $\mathrm{LaAlO}_{3}, \mathrm{LuLaO}_{3}, \mathrm{GdScO}_{3}, \mathrm{DyScO}_{3}, \mathrm{LaScO}_{3}, \ldots$, cf. Table 2 in [21]) which are considerably higher than some optical absorption edge energies reported in [98]. In the forthcoming analysis only these IPE results will be used to compare interface band alignments at different Si/oxide interfaces.

Finally, it needs to be mentioned that the carrier injection in RE oxides may be facilitated by the RE f-states in the O2p$\mathrm{RE} 5 \mathrm{~d}(6 \mathrm{~s})$ gap as has been revealed by both electron and hole IPE at the Si/ $\mathrm{Nd}_{2} \mathrm{O}_{3}$ interface [105]. The involvement of f-states complicates the picture further and, to avoid misunderstanding, we will consider RE oxide bandgap as the energy gap between the O2p-derived VB top edge and the oxide CB bottom originating from unoccupied RE $5 \mathrm{~d}(6 \mathrm{~s})$ states since this gap retains its width even if partially occupied f-orbitals appear within it.

The $Y^{1 / 3}-h v$ plots shown in Figure 12 illustrate the typical behavior of the electron IPE spectra at interfaces of silicon with $\mathrm{RE}$ and complex oxides of different composition using the case of $\mathrm{Gd}_{x} \mathrm{Al}_{2-x} \mathrm{O}_{3}$ layers with different $\mathrm{Gd}$ content grown by ALD. It is obvious that no measurable shift in the electron IPE threshold can be found despite the gap narrowing from $\approx 6 \mathrm{eV}$ in $\mathrm{Al}_{2} \mathrm{O}_{3}$ to the range close to $5.5 \mathrm{eV}$ in pure $\mathrm{Gd}_{2} \mathrm{O}_{3}$, as revealed by the PC spectra shown for the same samples in Figure 11. This observation suggests that the energy of the oxide $\mathrm{CB}$ bottom is marginally sensitive to the composition of the oxide.

The same conclusion can also be made for the ALDgrown $\mathrm{Lu}_{x} \mathrm{Al}_{2-x} \mathrm{O}_{3}$ insulators on the basis of the PC and electron IPE spectra shown in Figures 13 and 14, respectively. The PC spectra reveal no substantial bandgap variation when 


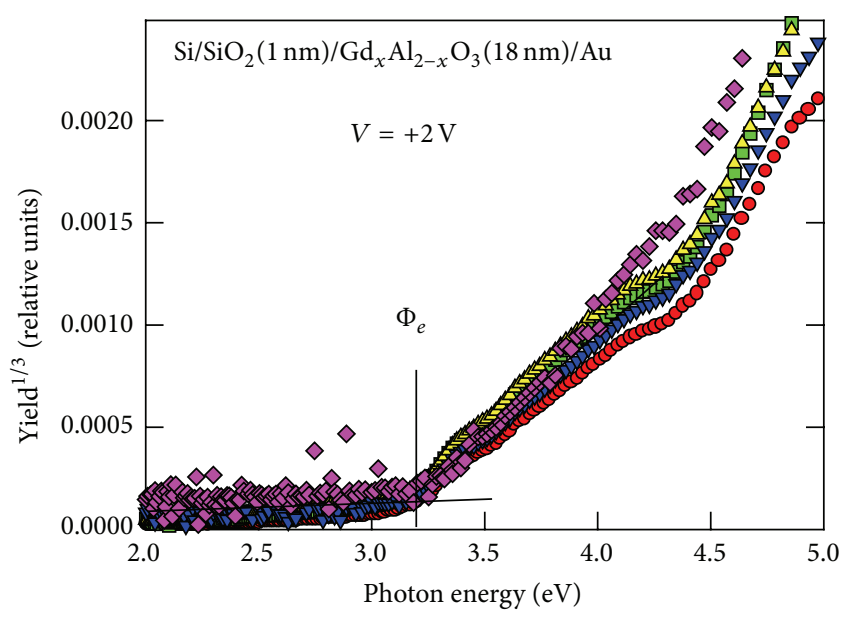

[Gd] (\%)

$\begin{array}{lll}\nabla & 0 & \Delta 70 \\ \circ 25 & \diamond 100 \\ \square 50 & \end{array}$

FIGURE 12: Cube root of the IPE yield as a function of photon energy plot used to find the spectral threshold $\Phi_{e}$ of electron IPE from the $\mathrm{VB}$ of $\mathrm{Si}$ into the $\mathrm{CB}$ of $\mathrm{Gd}_{x} \mathrm{Al}_{1-x} \mathrm{O}_{3}$ is indicated by vertical line.

changing the oxide composition. However, the photoconductivity is seen to increase significantly upon crystallizing the film by postdeposition annealing (PDA) at $1000^{\circ} \mathrm{C}$. Remarkably though, the already mentioned "tail" in the PC spectrum appears to be enhanced despite a lower volume fraction of the remaining amorphous phase. An even more significant impact of crystallization on the electron IPE spectra is seen in Figure 14. While no signal which may be potentially identified as IPE from silicon can be found in the as-deposited amorphous sample, upon PDA the spectral curves emerge which bear all features of the IPE from Si. These spectra closely resemble the electron IPE spectrum from Si into a$\mathrm{Al}_{2} \mathrm{O}_{3}$ shown on the same graph for comparison. Again, the same spectral threshold of IPE close to $3.1 \mathrm{eV}$ is found for all oxide compositions.

Several other results from previous studies are also worth being listed.

(i) There is no measurable difference between the band offsets encountered at interfaces of Si with amorphous and crystalline RE oxides. Only the "tail" portions in the PC and IPE spectra are affected.

(ii) Variation of silicon surface crystallographic orientation from the traditional (100) to (111) face which has a higher surface atom density has no measurable effect on the interface band diagram.

(iii) Atomically abrupt interfaces have the same band alignment as those with "inserted" $\approx 1 \mathrm{~nm}$ thick $\mathrm{SiO}_{2}$ IL.

(iv) There is no measurable variation of the band offsets due to differences in composition of the interfacial layer, for example, between silicate-like and oxide-like $\mathrm{Gd}_{2} \mathrm{O}_{3} / \mathrm{Si}$ interfaces [106].
As the final step, the available up to date values of Si/oxide CB and VB offsets inferred from the IPE and PC experiments are compiled in Figure 15 in which they are shown as functions of the ionic radius of the cation in the corresponding oxide. Open circles and squares correspond to the $\mathrm{CB}$ and $\mathrm{VB}$ edge energies in amorphous oxides while the filled ones show the same energies in the crystallized (cubic) oxides. Red and green symbols show the $\mathrm{CB}$ and $\mathrm{VB}$ energies in the oxides for which no comparison between amorphous and the crystalline phases is possible yet. The points for $\mathrm{Ti}^{4+}$ cation are placed using the earlier indicated XPS results [87]. The compiled band offset results indicate the remarkable stability of the VB top energy, within a $\pm 0.2 \mathrm{eV}$ interval, in oxides of cations with ionic radius $r_{i}$ exceeding 0.75-0.8 $\AA$ as well as for $\mathrm{Ti}^{4+}$ and $\mathrm{Ta}^{5+}$ with no noticeable sensitivity to the oxide crystallinity. Furthermore, in the case of amorphous films, oxides with even smaller cations appear to have the VB energies in the indicated range. This observation suggests that the $\mathrm{O} 2 \mathrm{p}$-derived electron states near the top of the oxide $\mathrm{VB}$ become nearly insensitive to the cation type and the details of the oxide network structure if the cation is large enough. Only in the case of small cations, substantial variations of the bandgap edges as affected by the oxide composition and crystallinity are observed. Taking into account that most of the oxides of interest as high- $\kappa$ insulators correspond to the large cation radii, the revealed simple band offset scheme with constant VB top energy interval of $2.5 \pm$ $0.2 \mathrm{eV}$ below the $\mathrm{VB}$ of silicon allows one to evaluate the band offset simply from the oxide bandgap width. In most of the cases, the oxide charge density in the studied samples has been controlled using capacitance-voltage measurements and revealed that the density of charged centers does not exceed $\approx 3 \times 10^{12} \mathrm{~cm}^{-2}$. Therefore, the Coulomb perturbation of the interface barrier cannot be kept responsible for the band offset variations as a function of interface structure and composition. Apparently then, the indicated \pm 0.2 width of the oxide VB energy interval reflects the typical value of interface dipole variation at the studied Si/oxide interfaces.

\section{Band Alignment at Interfaces of Germanium and Ge-Based Semiconductors}

Germanium attracts large interest as the possible channel material primarily because of its intrinsically high hole mobility. However, this semiconductor lacks a stable native oxide which can be used as gate insulator since $\mathrm{GeO}_{2}$ is known to be water-soluble and prone to degradation at an elevated temperature. As a result, most of the attention has been devoted to the search for a suitable nonnative insulator as well as to the development of Ge surface passivation schemes which would allow one to attain a low density of Ge/oxide interface traps. Furthermore, the $\mathrm{Ge}_{x} \mathrm{Si}_{1-x}$ and $\mathrm{Sn}_{x} \mathrm{Ge}_{1-x}$ alloys were also proposed for strained channel devices which raised an interest to study their interfaces with different oxide insulators. In this section, we will overview the available IPE results concerning the band alignment at interfaces of $\mathrm{Ge}$ with several insulating oxides. 


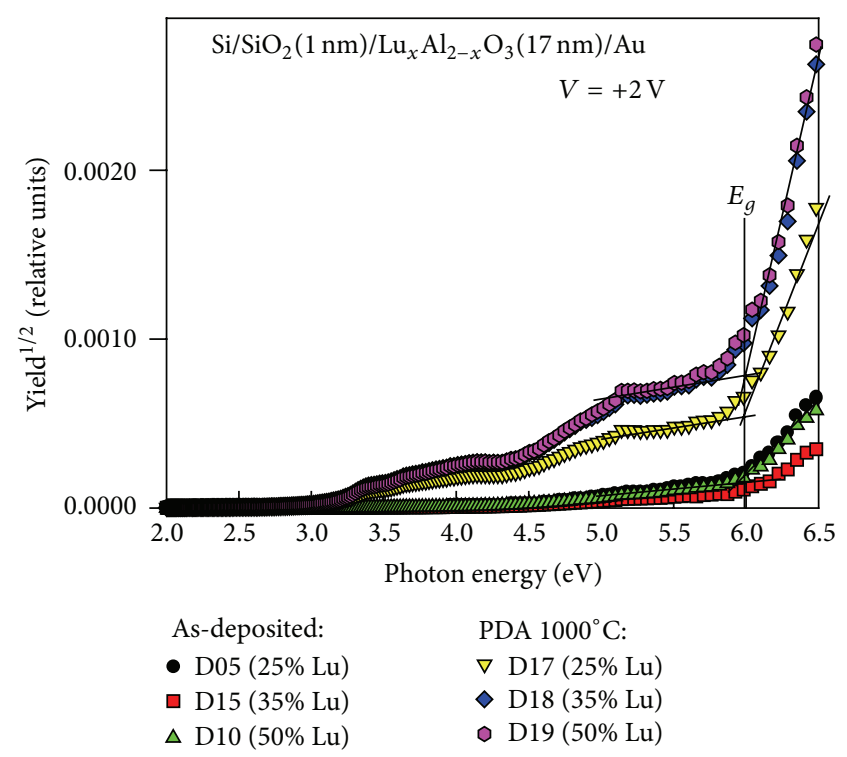

(a)

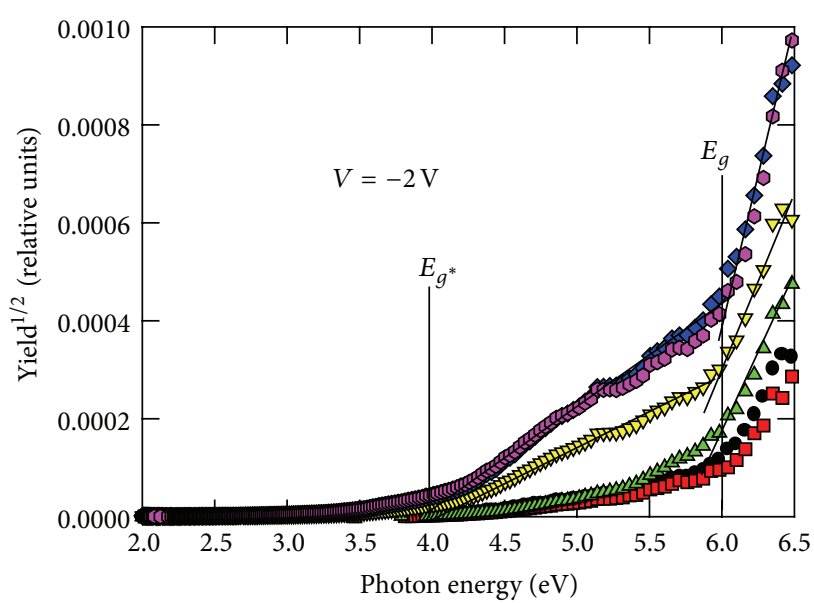

As-deposited:

- D05 (25\% Lu)

- D15 $(35 \% \mathrm{Lu})$

$\triangle \mathrm{D} 10(50 \% \mathrm{Lu})$
$\operatorname{PDA} 1000^{\circ} \mathrm{C}$ :

$\nabla \mathrm{D} 17(25 \% \mathrm{Lu})$

$\diamond \mathrm{D} 18(35 \% \mathrm{Lu})$

- D19 $(50 \% \mathrm{Lu})$

(b)

Figure 13: Square root of the PC yield as a function of photon energy in $\mathrm{Si} / \mathrm{SiO}_{2} / \mathrm{Lu}_{x} \mathrm{Al}_{1-x} \mathrm{O}_{3} / \mathrm{Au}$ capacitors with different concentration of lutetium in the insulating layer as measured under positive (a) and negative (b) bias of $2 \mathrm{~V}$ applied to the top Au electrode. The results are shown both for the as-deposited samples as for those supplementally annealed at $1000^{\circ} \mathrm{C}$ in $\mathrm{N}_{2}$ for 1 min prior to metallization. The inferred spectral thresholds of the intrinsic PC are indicated by vertical lines $E_{g}$ and $E_{g^{*}}$.

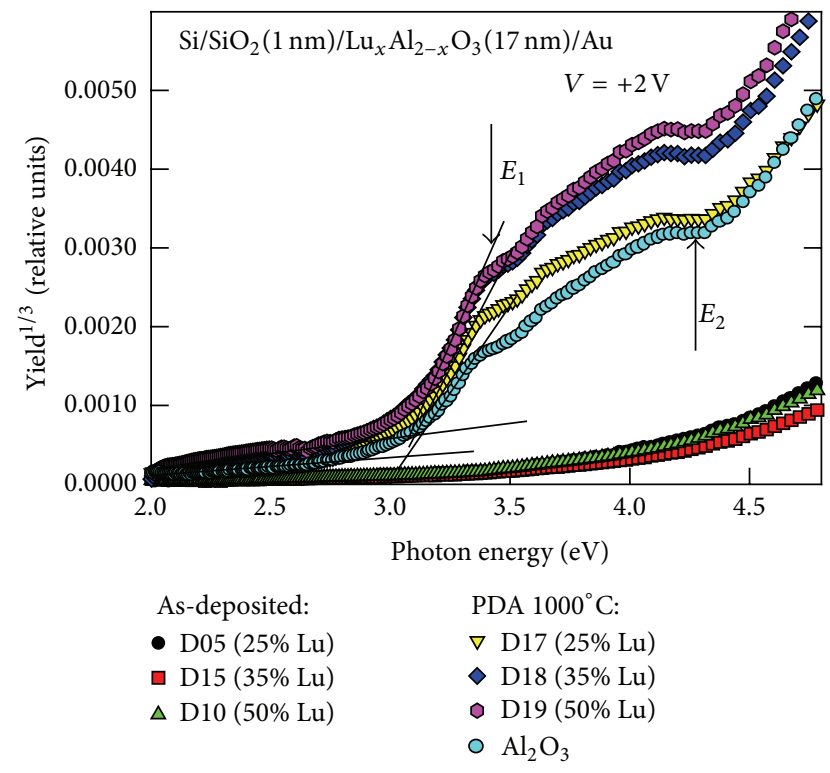

Figure 14: Cube root of the IPE yield as a function of photon energy as inferred from the data shown in Figure 13. The inferred spectral threshold $\Phi_{e}$ of electron IPE from the VB of Si into the CB of $\mathrm{Lu}_{x} \mathrm{Al}_{1-x} \mathrm{O}_{3}$ is indicated by vertical line. The arrows $E_{1}$ and $E_{2}$ indicate onsets of direct optical transition in the silicon crystal.

6.1. Ge/Oxide Interfaces. The major difference between interfaces of germanium with a deposited oxide from the similar $\mathrm{Si} /$ oxide interface consists in the fast oxidation of the Ge surface resulting in a thin $(\approx 1-2 \mathrm{~nm})$ IL of Ge oxide. Unlike

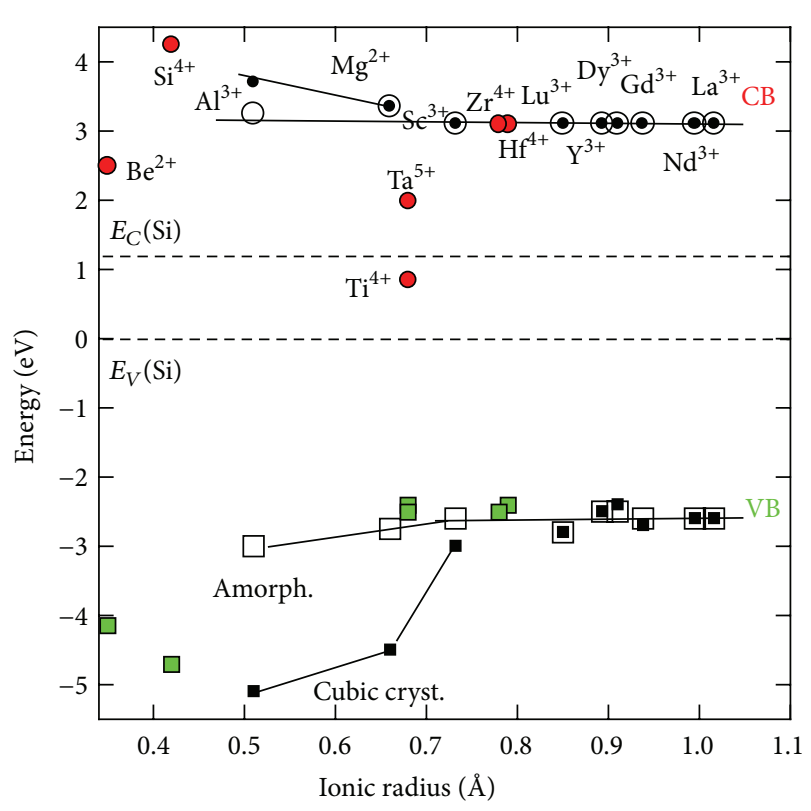

FIgURE 15: Energies of the VB top and CB bottom in different oxide insulators as a function of metal cation radius referenced to the band edges of the Si crystal as inferred from the IPE experiments.

the wide-gap $\mathrm{SiO}_{2}$ ILs, the Ge oxide grown during ALD or $\mathrm{MBD}$ of a metal oxide is characterized by a relatively narrow bandgap of $4.4 \mathrm{eV}$ which leads to the lower thresholds of electron and hole injection [106-109]. As a result, the IPE spectra of Ge/oxide samples usually exhibit two additional contributions which are exemplified in Figure 16 for the case 


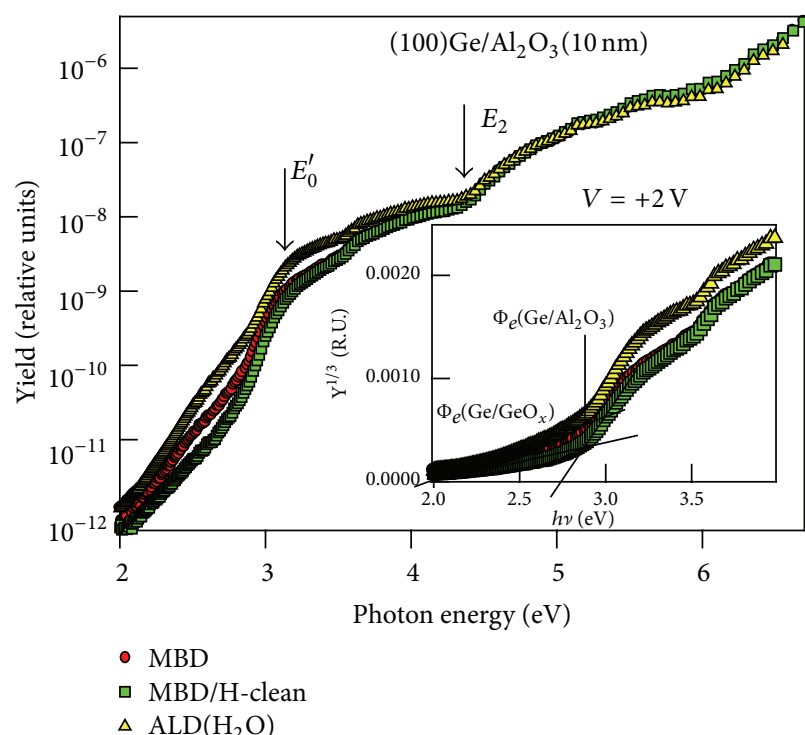

(a)

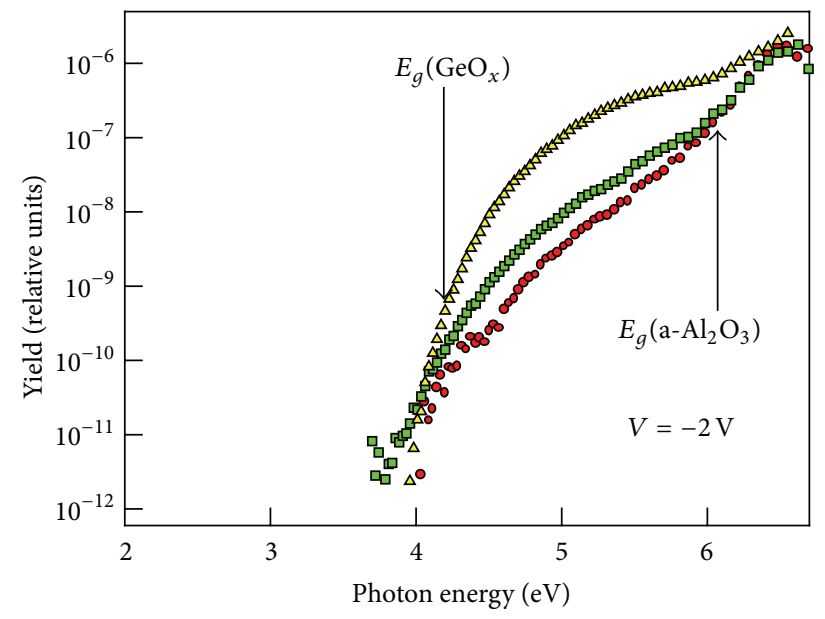

- MBD

口 $\mathrm{MBD} / \mathrm{H}$-clean

$\triangle \operatorname{ALD}\left(\mathrm{H}_{2} \mathrm{O}\right)$

(b)

FIGURE 16: Semilogarithmic plot of the photocurrent yield spectra of (100)Ge/a- $\mathrm{Al}_{2} \mathrm{O}_{3}(10 \mathrm{~nm}) / \mathrm{Au}$ capacitors fabricated using different alumina deposition methods as measured under (a) positive and (b) negative bias of 2 Volt applied to the top metal electrode. The inset in panel (a) illustrates determination of the electron IPE spectral thresholds using $Y^{1 / 3}-h v$ plots. The threshold energies $\Phi_{e}$ are indicated for electron transitions from the $\mathrm{VB}$ of germanium into the $\mathrm{CB}$ of $\mathrm{a}-\mathrm{Al}_{2} \mathrm{O}_{3}$ and into the $\mathrm{CB}$ of $\mathrm{GeO}_{x}$ IL formed during oxide deposition. The vertical arrows $E_{0}^{\prime}$ and $E_{2}$ in panel (a) mark the energies of direct optical transitions in the Ge crystal. In panel (b) the vertical arrows mark the onsets of the photoconductivity in a- $\mathrm{Al}_{2} \mathrm{O}_{3}$ film and $\mathrm{GeO}_{x} \mathrm{IL}$.

of $\mathrm{Al}_{2} \mathrm{O}_{3}$ grown on Ge using MBD or ALD techniques. The electron IPE spectra reveal two thresholds corresponding to the direct IPE into the CB of $\mathrm{Al}_{2} \mathrm{O}_{3}\left(\Phi_{e}\left(\mathrm{Ge} / \mathrm{Al}_{2} \mathrm{O}_{3}\right)=\right.$ $2.85 \pm 0.10 \mathrm{eV})$ or to transitions mediated by the $\mathrm{GeO}_{x} \mathrm{IL}\left(\Phi_{e}\right.$ $\left.\left(\mathrm{Ge} / \mathrm{GeO}_{x}\right)=2.1 \pm 0.2 \mathrm{eV}\right)$ as shown in panel (a). In turn, the PC spectra shown in panel (b) indicate two thresholds corresponding to the electron-hole pair generation in $\mathrm{GeO}_{x}$ $\mathrm{IL}$ and in the bulk of a- $\mathrm{Al}_{2} \mathrm{O}_{3}$. The oxide deposition method strongly affects the $\mathrm{GeO}_{x}$ IL thickness leading to the well detectable process-induced variations of the IPE or PC yield. Nevertheless, the same electron IPE threshold from the VB of (100)Ge into the CB of Ge oxide is found both for deposited [110] and thermally grown [111] $\mathrm{GeO}_{2}$ layers.

As compared to the interfaces of silicon, the $\mathrm{VB}$ of $\mathrm{Ge}$ is up-shifted in energy by $0.4-0.45 \mathrm{eV}$ while the $\mathrm{CB}$ bottom remains nearly at the same energy as in Si. This semiconductor VB shift can directly be seen when comparing the electron IPE spectra for Si and Ge photoemitters shown in Figure 17 for the same insulator $\mathrm{La}_{2} \mathrm{Hf}_{2} \mathrm{O}_{7}$. By contrast, the absence of any measurable shift of the hole IPE threshold indicates the same $\mathrm{CB}$ position of $\mathrm{Si}$ and $\mathrm{Ge}$ with respect to the reference level of the oxide VB top. The spectra shown in Figure 17 also reveal a much reduced signal from the ILassisted electron IPE which reflects a transformation of the IL from $\mathrm{GeO}_{x}$ into a wide-gap La germanate which allows one to suppress low-field electron injection in the oxide stack $[112,113]$. Among other methods enabling reduction of the $\mathrm{Ge}$ oxidation one may mention the Ge surface passivation with several monolayers of silicon or using a $\mathrm{GeN}_{x}$ barrier layer $[114,115]$. In all these cases no IL-sensitive or crystallographic

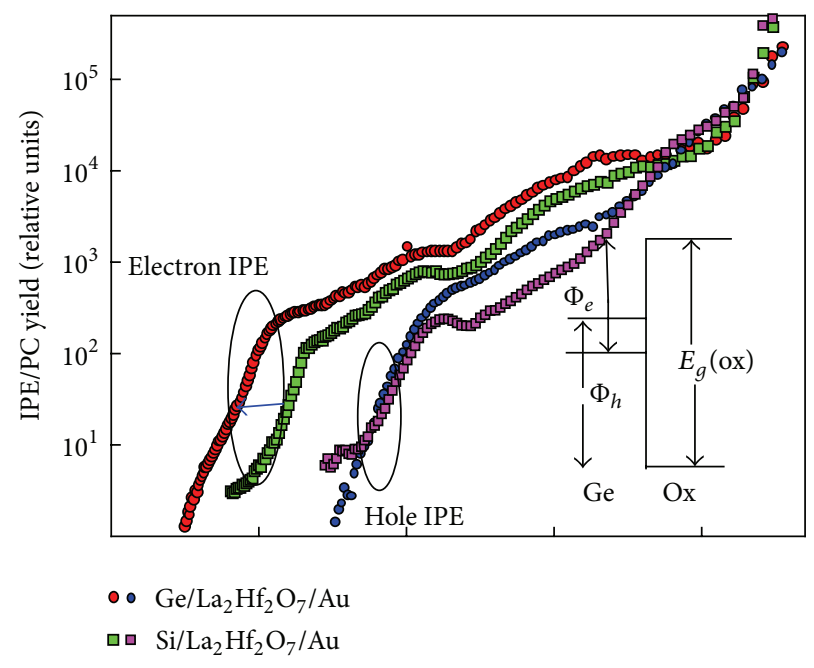

FIGURE 17: Semilogarithmic plots comparing electron and hole IPE spectra from $\mathrm{Si}$ and $\mathrm{Ge}$ into a $10 \mathrm{~nm}$ thick $\mathrm{La}_{2} \mathrm{Hf}_{2} \mathrm{O}_{7}$ insulator. The inset schematically shows the interface band diagram and illustrates the physical meaning of the observed spectral thresholds.

face related variations of the Si/high- $\kappa$ oxide band offsets have been found affirming the behavior earlier found at interfaces of silicon with insulating metal oxides.

Summarizing the available results regarding band offsets at the interfaces of Ge with insulating metal oxides [106118 ], the energies of the oxide $\mathrm{VB}$ and $\mathrm{CB}$ edges are shown in 


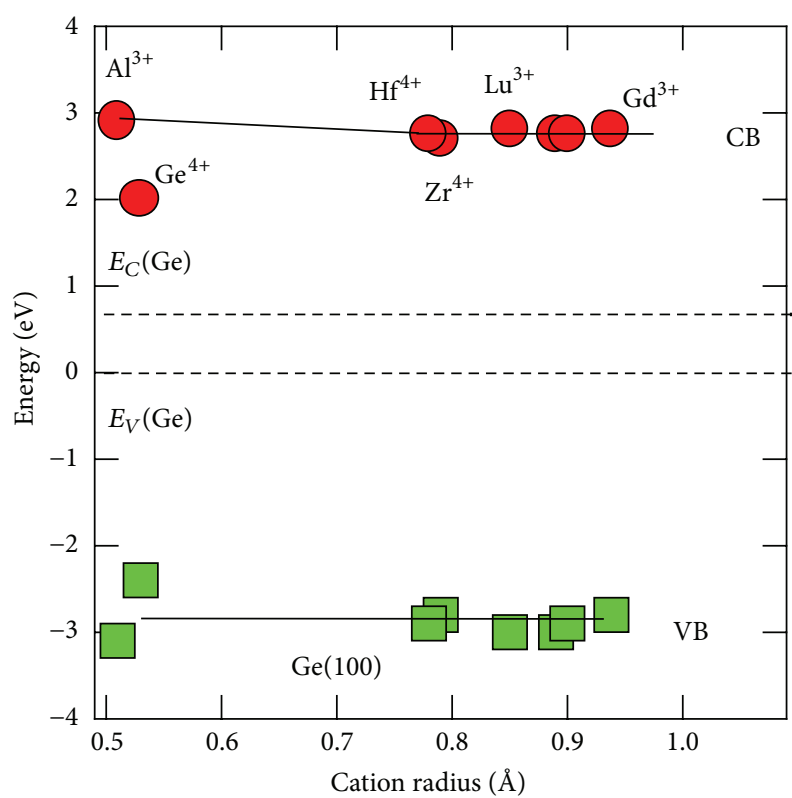

FIGURE 18: Energies of the VB top and CB bottom in different oxides as a function of cation radius referenced to the band edges for $\mathrm{Ge}$ crystal as inferred from the IPE experiments. The top of the Ge VB is at $\delta E_{V}=0.4 \mathrm{eV}$ above the silicon $\mathrm{VB}$ as measured relative to the common a- $\mathrm{Al}_{2} \mathrm{O}_{3} \mathrm{CB}$ bottom reference level.

Figure 18 as functions of the corresponding cation radius. The origin of the energy scale is placed at the top of the $\mathrm{Ge} \mathrm{VB}$, that is, at $0.4 \mathrm{eV}$ above the $\mathrm{VB}$ of silicon (cf. Figure 17). The cations of large radii are all seen to provide the $\mathrm{VB}$ offset of $2.9 \mathrm{eV}$. For the $\mathrm{Al}^{3+}$ and $\mathrm{Ge}^{4+}$ cations the oxide $\mathrm{VB}$ is also positioned at close energy. If referenced to the Si bandgap edge positions, the $2.9 \mathrm{eV}$ offset with $\mathrm{VB}$ of Ge would become a $2.5 \mathrm{eV}$ offset with respect to the VB of Si thus yielding the same "universal" value as shown in Figure 15. Therefore, one may conclude that the energy of the O2p-derived VB in insulating oxides is reproduced irrespective of the semiconductor substrate crystal. We will further support this result by comparing band offsets of GaAs with different insulators.

6.2. Interfaces of $\mathrm{Ge}_{x} \mathrm{Si}_{1-x}$ with Thermal $\mathrm{SiO}_{2}$. Analysis of band offsets at interfaces of $\mathrm{Si}_{1-x} \mathrm{Ge}_{x}$ alloys with insulating materials offers a unique opportunity to systematically investigate the influence of Ge concentration on the bandgap edges. In the previous studies, comparing $\mathrm{Si} / \mathrm{SiO}_{2}$ and $\mathrm{SiC} / \mathrm{SiO}_{2}$ interfaces the predominant effect of carbon incorporation has been found to be a considerable $(1.75 \mathrm{eV})$ $\mathrm{VB}$ downshift while the $\mathrm{CB}$ bottom in $\mathrm{SiC}$ remains in the vicinity of the $\mathrm{CB}$ of silicon though exhibiting some SiC-polytype sensitive variations [119-122]. In the case of $\mathrm{Si}_{1-x} \mathrm{Ge}_{x}$, the interface with the same reference insulator (thermally grown $\mathrm{SiO}_{2}$ ) can be fabricated using the condensation growth method in which Ge concentration in the $\mathrm{Si}_{1-x} \mathrm{Ge}_{x}$ is increased by oxidizing a thin layer of this material at high temperature $[123,124]$. By observing electron IPE from the $\mathrm{VB}$ of $\mathrm{Si}_{1-x} \mathrm{Ge}_{x}$ into the $\mathrm{CB}$ of $\mathrm{SiO}_{2}$, one can directly monitor the composition-dependent VB top energy [125].
As an example, in Figure 19 are shown the spectra of electron IPE from $\mathrm{Si}_{0.72} \mathrm{Ge}_{0.28}$ into the $\mathrm{CB}$ of an $83 \mathrm{~nm}$ thick top $\mathrm{SiO}_{2}$ layer measured at the indicated positive voltages applied to the Al gate electrode. The onset energy of IPE from the VB is observed as an abrupt increase of the photocurrent in the photon energy range $3<h \nu<4 \mathrm{eV}$ in Figure 19(a). Using the $Y^{1 / 3}-h \nu$ plots, the value of the corresponding spectral threshold $\Phi$ can be found for each strength $F$ of the externally applied electric field over the top oxide, as illustrated in Figure 19(b). The data clearly reveal a red shift of this IPE threshold with increasing electric field strength caused by the image-force barrier lowering (the Schottky effect). In Figure 19(a) and in the inset in Figure 19(b) one may also notice a photocurrent in the low-energy portion of the spectra $(h v<3 \mathrm{eV})$. A similar photocurrent spectrum is observed in the case of reversed electric field orientation in the oxide (curves not shown). This suggests a bulk electronic excitation in the oxide, possibly caused by the incorporation of some Ge atoms into the $\mathrm{SiO}_{2}$ network. However, this current has a very low quantum yield and, therefore, does not impair the accuracy of determination of the $\mathrm{Si}_{1-x} \mathrm{Ge}_{x} \mathrm{VB}$ energy.

In order to determine the correct energy offset between the top of the $\mathrm{Si}_{1-x} \mathrm{Ge}_{x} \mathrm{VB}$ and the bottom of the oxide CB we used linear extrapolation of the field-dependent spectral thresholds of the electron IPE to zero electric field using the Schottky coordinates $\Phi(F)-F^{1 / 2}$, as illustrated in Figure 20 for samples with different $\mathrm{Ge}$ fraction in the $\mathrm{Si}_{1-x} \mathrm{Ge}_{x}$ layer. The results are seen to obey the Schottky law quite well, suggesting that Ge has no noticeable influence on the oxide optical polarizability and does not introduce a significant density of bulk oxide charges. This observation suggests that the electric field possibly induced by the above mentioned electron states (traps) in $\mathrm{SiO}_{2}$ is negligible as compared to the externally applied field.

The inferred zero-field barrier height corresponding to the energy difference between the bottom of the $\mathrm{SiO}_{2} \mathrm{CB}, E_{C}$ $\left(\mathrm{SiO}_{2}\right)$, and the top of the $\mathrm{Si}_{1-x} \mathrm{Ge}_{x} \mathrm{VB}, E_{V}(\mathrm{SiGe})$, is shown as a function of Ge content in Figure 21. The decrease in the height of the barrier indicates that the top of the $\mathrm{Si}_{1-x} \mathrm{Ge}_{x}$ VB shifts up with increasing Ge content. This shift may be described by the linear function $E_{V}(\mathrm{eV})=(0.55 \pm 0.05) x$. Interestingly, this relationship corresponds to a $0.55 \mathrm{eV}$ shift between VBs of pure Si and pure Ge, which is by 0.10 $0.15 \mathrm{eV}$ larger than the difference between the VB tops of $\mathrm{Si}$ and $\mathrm{Ge}$ at the interfaces with deposited metal oxides $\left(\mathrm{Al}_{2} \mathrm{O}_{3}, \mathrm{HfO}_{2}\right)$. The possible explanation of this effect may be related to compressive strain developed in the thin $\mathrm{Si}_{1-x} \mathrm{Ge}_{x}$ layer during condensation growth [124]. Nevertheless, these results affirm the trend of predominant VB shift in the group IV semiconductor alloys though the effect of $\mathrm{Ge}$ addition to silicon appears to be much smaller than that of carbon.

6.3. Interfaces of $\mathrm{Sn}_{x} \mathrm{Ge}_{1-x}$ with $a-\mathrm{Al}_{2} \mathrm{O}_{3}$. To further investigate the band evolution trends in $\mathrm{A}_{\mathrm{IV}} \mathrm{B}_{\mathrm{IV}}$ alloys, we compared band offsets at the interfaces of $\mathrm{Ge}$ and $\mathrm{Sn}_{x} \mathrm{Ge}_{1-x}(0.04<$ $x<0.08)$ with deposited a- $\mathrm{Al}_{2} \mathrm{O}_{3}$. The IPE analysis of 


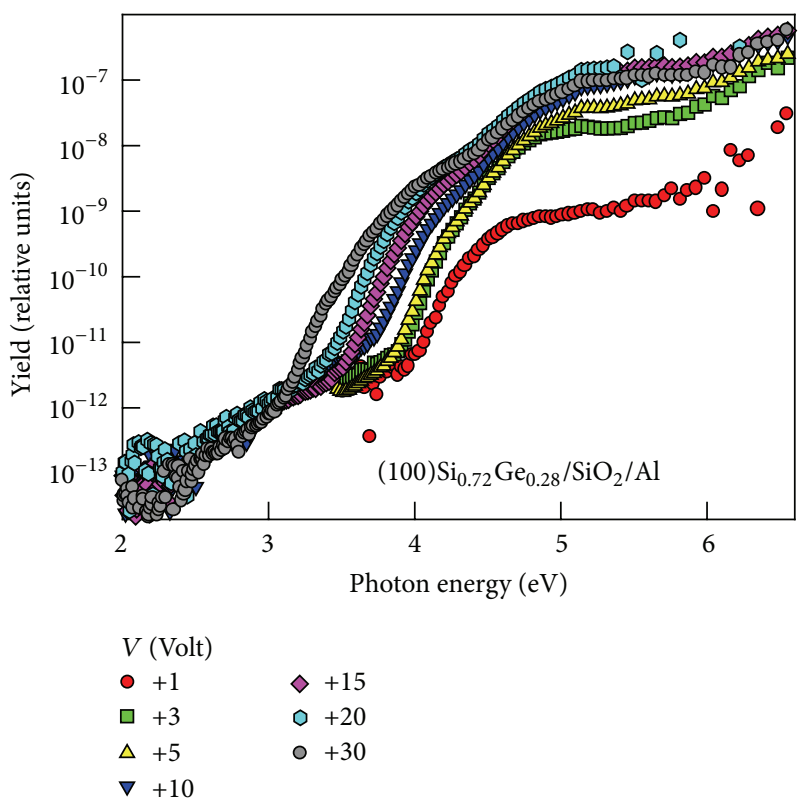

(a)

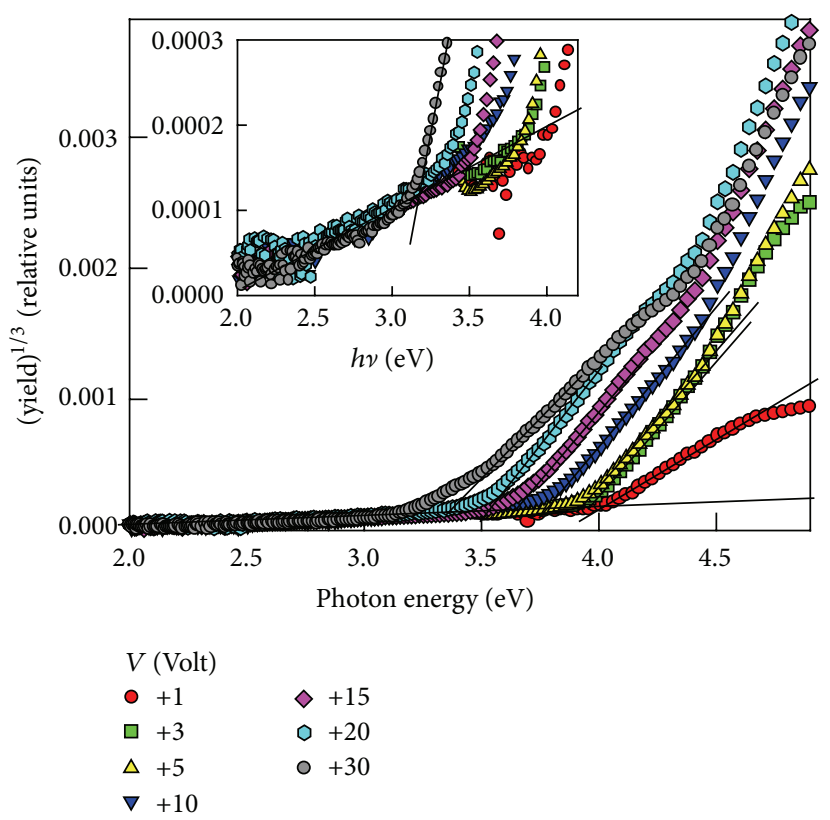

(b)

Figure 19: (a) Electron IPE quantum yield as a function of photon energy measured on $(100) \mathrm{Si}_{0.72} \mathrm{Ge}_{0.28} / \mathrm{SiO}_{2}(83 \mathrm{~nm}) / \mathrm{Al}$ samples under indicated positive bias on the $\mathrm{Al}$ gate electrode. (b) Determination of the electron IPE threshold $\Phi\left(\mathrm{Si}_{1-x} \mathrm{Ge}_{x}\right)$ using $Y^{1 / 3}-h \nu$ plots. Lines guide the eye. The inset shows a magnified view of the photocurrent yield in the subthreshold spectral region.

these interfaces represents a significant challenge because indiffusion of tin into the oxide causes high leakage current making the IPE analysis impossible. To resolve this issue, the alumina layers were grown by $\mathrm{MBD}$ at a low temperature (293 or $223 \mathrm{~K}$ as compared by standard $300^{\circ} \mathrm{C}$ in the ALD process) or, else, the $\mathrm{Sn}_{x} \mathrm{Ge}_{1-x}$ layer was capped by a $5 \mathrm{~nm}$ thick Ge layer [126]. The electron IPE spectra from a $9 \mathrm{~nm}$ thick $\mathrm{Sn}_{0.05} \mathrm{Ge}_{0.95}$ layer grown on top of (100)Ge substrate crystal into $9 \mathrm{~nm}$ thick a- $\mathrm{Al}_{2} \mathrm{O}_{3}$ are shown in Figure 22 as measured under different positive voltages applied to the top $\mathrm{Au}$ electrode. The spectra are very similar to those observed at the $\mathrm{Ge} / \mathrm{Al}_{2} \mathrm{O}_{3}$ interface (cf. Figure 16) and suggest onset of the direct electron IPE into the CB of alumina to be at around $2.85 \mathrm{eV}$. In higher fields, one observes the development of the $\mathrm{GeO}_{x}$ IL-related "tail" stretching to the photon energies close to $2 \mathrm{eV}$.

In order to evaluate the influence of $\mathrm{Sn}$ incorporation of the semiconductor VB energy in Figure 23 we compared the electron IPE spectra measured on $\mathrm{Ge} / \mathrm{a}-\mathrm{Al}_{2} \mathrm{O}_{3}$ sample and three $\mathrm{Sn}_{0.05} \mathrm{Ge}_{0.95} / \mathrm{a}-\mathrm{Al}_{2} \mathrm{O}_{3}$ samples prepared by $\mathrm{MBD}$ of alumina at low temperature, with or without removal of the native oxide from the $\mathrm{Sn}_{0.05} \mathrm{Ge}_{0.95}$ surface. In all cases, the electron IPE threshold $\Phi_{e}$ appears to be at the same energy of $2.9 \mathrm{eV}$, indicating that incorporation of $\mathrm{Sn}$ does lead to any measurable VB shift. Affirming the correctness of the above conclusion, the same result has also been obtained in the case of $\mathrm{Sn}_{0.08} \mathrm{Ge}_{0.92}$ layers irrespective of the Ge substrate induced strain or the presence of a $5-3 \mathrm{~nm}$ thick Ge capping layer. Therefore, the $0.1-0.15 \mathrm{eV}$ bandgap narrowing expected to occur in $\mathrm{Sn}_{x} \mathrm{Ge}_{1-x}$ alloys for $0.05<x<0.08$ must be occurring in the $\mathrm{CB}$ side of the $\mathrm{Sn}_{x} \mathrm{Ge}_{1-x}$ bandgap. These results suggest the reversal of the trend of the predominant shift of the semiconductor VB earlier observed upon C or Ge incorporation into silicon.

\section{Band Alignment at Interfaces of $\mathbf{A}_{\mathrm{III}} \mathbf{B}_{\mathrm{V}}$ Semiconductors with Insulating Oxides}

Compound semiconductors of $\mathrm{A}_{\mathrm{III}} \mathrm{B}_{\mathrm{V}}$ group are attracting great technological interest as the materials with record high electron mobility providing the possibility of quantum well/wire channel engineering as well as fabrication of tunneling structures and optoelectronic devices. Here we are not addressing the technological issues but will mostly focus on the evolution of band alignment as affected by the composition of the $\mathrm{A}_{\mathrm{III}} \mathrm{B}_{\mathrm{V}}$ material by comparing band offsets of arsenides, phosphides, and antimonides of In and $\mathrm{Ga}$. First, we will discuss the IPE results concerning band offsets at interfaces of GaAs with different insulators in order to check on the applicability of the common anion rule to the GaAs/oxide interfaces. This would enable one to use a much wider set of experimental data obtained at interfaces of silicon in order to evaluate the band alignment at interfaces of these oxides with GaAs. Next, we will turn towards the semiconductor side of the interface and discuss the effects caused by variation of the group $\mathrm{V}$ anion ( $\mathrm{P}, \mathrm{As}, \mathrm{Sb}$ ) or group III cation ( $\mathrm{Ga}, \mathrm{As}, \mathrm{Al})$ in the $\mathrm{A}_{\mathrm{III}} \mathrm{B}_{\mathrm{V}}$ compound as measured with respect to band edges of the "reference" insulator-a$\mathrm{Al}_{2} \mathrm{O}_{3}$. Finally, we will consider the IPE from semiconductor heterojunctions to demonstrate the applicability of the band offset transitivity rule. 


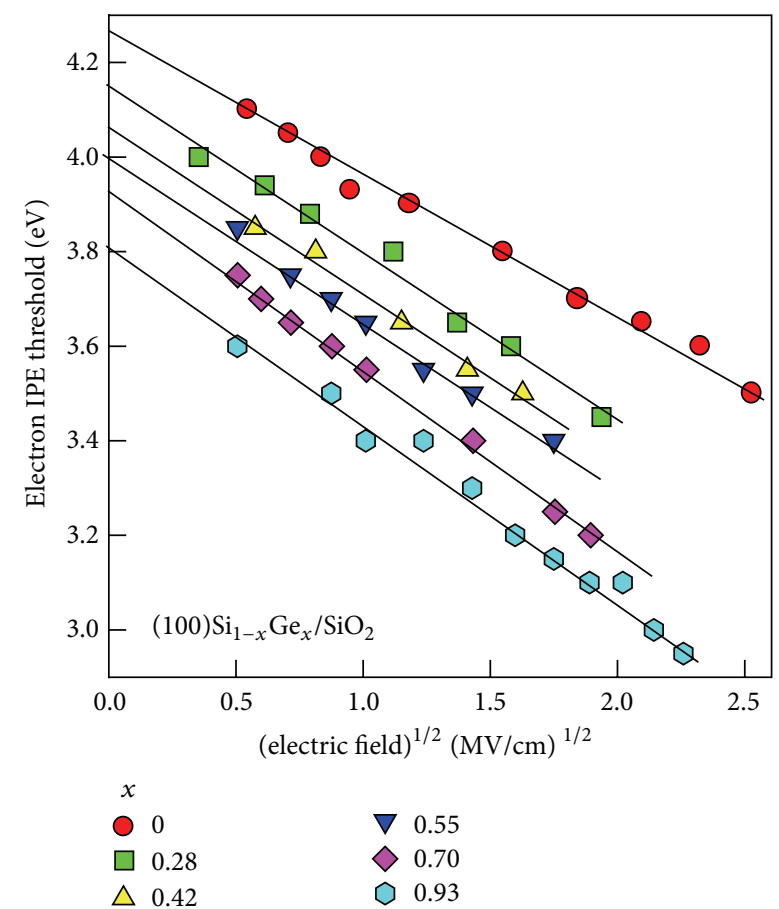

FIGURE 20: Schottky plot of the electron IPE spectral threshold from the $\mathrm{VB}$ of $\mathrm{Si}_{1-x} \mathrm{Ge}_{x}$ layers with different $\mathrm{Ge}$ content into the $\mathrm{CB}$ of thermal $\mathrm{SiO}_{2}$. Lines illustrate linear fits used to determine the zerofield barrier value.

7.1. Interfaces of Arsenides of In and $\mathrm{Ga}$ with Oxide Insulators. Among $\mathrm{A}_{\mathrm{III}} \mathrm{B}_{\mathrm{V}}$ semiconductors GaAs has enjoyed most of the attention being considered as the prototype material for MIS devices. As a result, band offsets at interfaces of GaAs with a considerable number of insulators have been experimentally determined which allows us to compare the energies of the oxide $\mathrm{CB}$ and $\mathrm{VB}$ edges measured with respect to the bandgap edges in the same GaAs photoemitter. Similar to the case of $\mathrm{Ge} /$ oxide interface, formation of a narrow gap IL represents the major difficulty in analyzing the IPE spectra, because this IL has a narrow bandgap with the width close to that of $\mathrm{Ga}_{2} \mathrm{O}_{3}(\approx 4 \mathrm{eV})$ leading to the additional IL-assisted IPE with low spectral threshold. The importance of the Ga-oxide IL influence was first recognized when studying interfaces in which the $\mathrm{Ga}_{2} \mathrm{O}_{3}$ IL was used as the passivation layer enabling one to achieve a low interface trap density $[127,128]$. Later, similar effects were encountered in the samples with ALD-grown $\mathrm{Al}_{2} \mathrm{O}_{3}$ and $\mathrm{HfO}_{2}[129,130]$. By combining the IPE with electron microscopy analysis it becomes possible to correlate the low-energy IPE threshold with the presence of a thicker IL between GaAs and the deposited high- $\kappa$ insulator [131]. The latter work has also importantly revealed that interfaces of $\mathrm{Al}_{2} \mathrm{O}_{3}$ with $\mathrm{GaAs}$ are much less prone to the IL growth than the interfaces between $\mathrm{GaAs}$ and $\mathrm{HfO}_{2}$ pointing towards the "cleaning" effect of trimetilaluminum $\mathrm{Al}\left(\mathrm{CH}_{3}\right)_{3}$ molecules used as the $\mathrm{Al}$ precursor in the ALD process $[132,133]$. By combining an optimized GaAs surface cleaning with the minimal IL growth it appears possible to fabricate samples exhibiting clear electron IPE thresholds

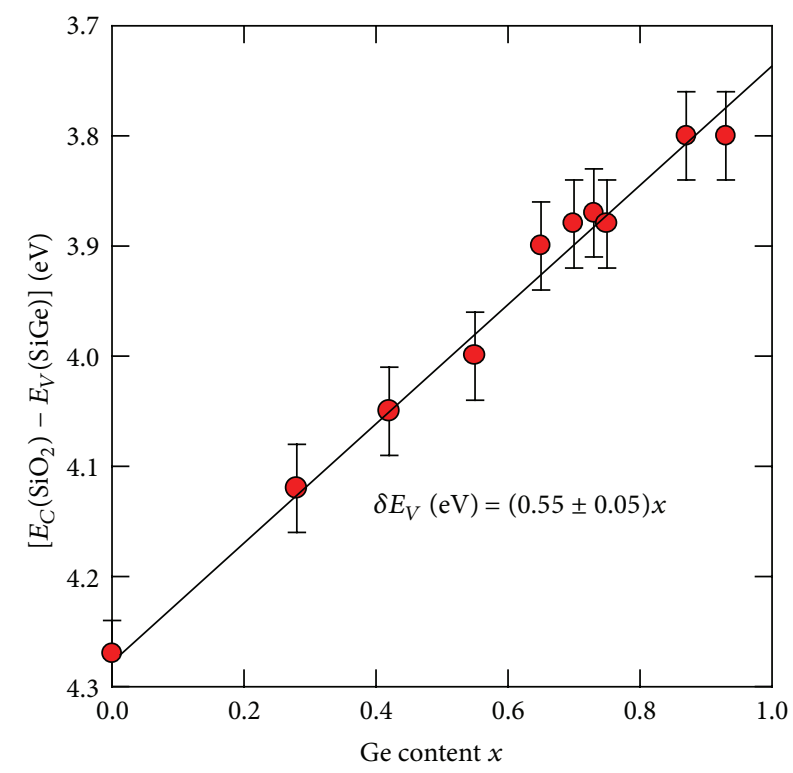

FIGURE 21: Energy barrier between the VB top of $\mathrm{Si}_{1-x} \mathrm{Ge}_{x}$ layers and the bottom of the $\mathrm{SiO}_{2} \mathrm{CB}$ as a function of Ge content in the semiconductor.

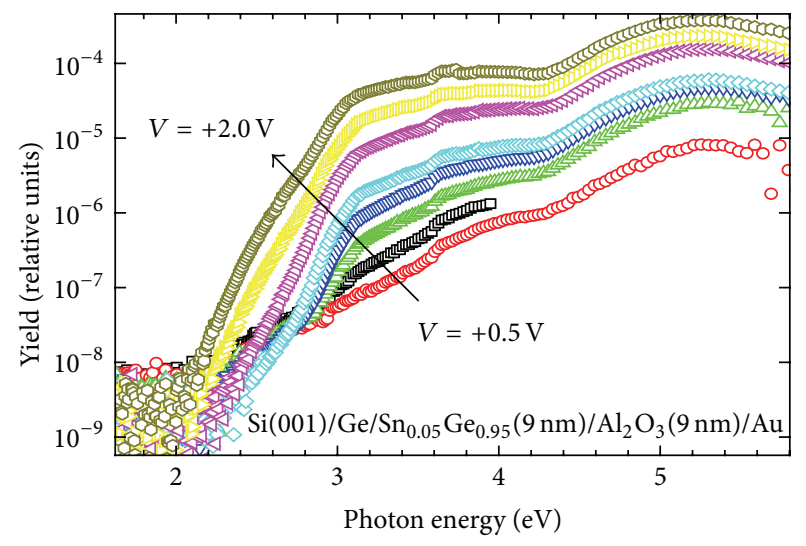

FIgURE 22: Semilogarithmic plot of the IPE quantum yield as a function of photon energy as measured on (100) $\mathrm{Si} / \mathrm{Ge} / \mathrm{Sn}_{0.05} \mathrm{Ge}_{0.95}(9 \mathrm{~nm}) / \mathrm{Al}_{2} \mathrm{O}_{3}(9 \mathrm{~nm}) / \mathrm{Au}$ sample with the applied bias varying from $0.5 \mathrm{~V}$ to $2.0 \mathrm{~V}$.

both at interfaces of $\mathrm{GaAs}$ with $\mathrm{Al}_{2} \mathrm{O}_{3}$ and $\mathrm{HfO}_{2}$. An example of such IPE spectra is presented in Figure 24 as $Y^{1 / 3}-h v$ plots for (100) $\mathrm{GaAs} / \mathrm{Al}_{2} \mathrm{O}_{3}$ and $(100) \mathrm{GaAs} / \mathrm{HfO}_{2}$ interfaces in panels (a) and (b), respectively. The red symbols correspond to the samples with the thinnest IL [131] and allow for reliable spectral threshold extraction. In the case of the $\mathrm{GaAs} / \mathrm{HfO}_{2}$ interface with a thick Ga-oxide IL (O-plasma assisted ALD, blue symbols in panel (b)), the additional low-energy IPE band becomes visible which is related to the IL-assisted electron photoinjection. Interestingly, despite different thickness of the IL, no additional field-induced shift in the spectral thresholds corresponding to direct IPE of electrons from $\mathrm{GaAs}$ into the $\mathrm{CB}$ of $\mathrm{Al}_{2} \mathrm{O}_{3}$ or $\mathrm{HfO}_{2}$ is 


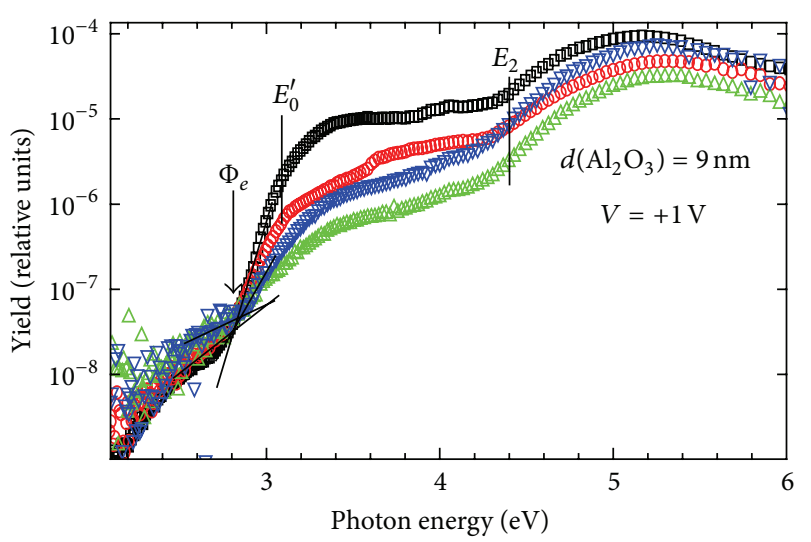

$$
\begin{array}{ll}
\square & \mathrm{Ge} / \mathrm{Al}_{2} \mathrm{O}_{3}(\mathrm{MBD}, 323 \mathrm{~K}) \\
\circ & \mathrm{Sn}_{0.05} \mathrm{Ge}_{0.95} / \mathrm{Al}_{2} \mathrm{O}_{3}(\mathrm{MBD}, 293 \mathrm{~K}) \\
\triangle & \mathrm{Sn}_{0.05} \mathrm{Ge}_{0.95} / \mathrm{Al}_{2} \mathrm{O}_{3}(\mathrm{MBD}, 223 \mathrm{~K}) \text {, native oxide } \\
\nabla & \mathrm{Sn}_{0.05} \mathrm{Ge}_{0.95} / \mathrm{Al}_{2} \mathrm{O}_{3}(\mathrm{MBD}, 223 \mathrm{~K})
\end{array}
$$

FIGURE 23: Semilogarithmic plot of the IPE quantum yield as a function of photon energy as measured on (100)Si/Ge/a$\mathrm{Al}_{2} \mathrm{O}_{3}(9 \mathrm{~nm}) / \mathrm{Au}$ and (100)Si/Ge/Sn $\mathrm{Sn}_{0.05} \mathrm{Ge}_{0.95} / \mathrm{a}-\mathrm{Al}_{2} \mathrm{O}_{3}(9 \mathrm{~nm}) / \mathrm{Au}$ samples prepared by using different alumina growth schemes.

observed supporting the earlier mentioned inference about projection of the oxide CB DOS across the IL.

Another important observation concerns the electron IPE barriers at the polar interfaces of GaAs. Direct comparison between (111)A (Ga-terminated) and (111)B (Asterminated) faces of GaAs covered with deposited a- $\mathrm{Al}_{2} \mathrm{O}_{3}$ reveals no electron barrier variation as compared to the (100) GaAs/a- $\mathrm{Al}_{2} \mathrm{O}_{3}$ interface [134]. This experiment indicates the absence of significant structure or composition-sensitive dipoles at GaAs interfaces, which is quite in contrast with the well known GaAs surface dipoles. The importance of this result consists in the possibility to use the interface barrier heights determined for (100)GaAs surface orientation to evaluate interface barriers at other faces which would not be possible in the case of different GaAs surfaces. The results regarding energies of the $\mathrm{CB}$ and $\mathrm{VB}$ edges of several studied high $-\kappa$ insulators are summarized in Figure 25 using the bandgap edges of the GaAs photoemitter as the reference levels. Alhough the selection of the studied oxides is considerably less rich than in the case of silicon (cf. Figure 15), the general trend of preserving the oxide VB energy irrespective of the oxide bandgap width is seen to be holding at the interfaces of GaAs as well. Observation of this behavior at interfaces of three different semiconductor materials ( $\mathrm{Si}, \mathrm{Ge}$, $\mathrm{GaAs}$ ) allows us to suggest that this behavior represents the universal trend at least for the oxides with sufficiently large cation radii $\left(r_{i}>0.07-0.08 \mathrm{~nm}\right)$.

Let us now briefly consider band alignment observed at the interfaces of GaAs, $\operatorname{In}_{x} \mathrm{Ga}_{1-x}$ As, and InAs with insulating a- $\mathrm{Al}_{2} \mathrm{O}_{3}$ [135-137]. The most straightforward way to trace the impact of replacing gallium by indium in the semiconductor is to directly compare the electron IPE spectra measured in the samples with identically prepared insulating layer. The example shown in Figure 26 for GaAs and $\mathrm{In}_{x} \mathrm{Ga}_{1-x}$ As layers of two compositions ( $x=0.15$ and $x=0.53$ ) allows one to estimate the difference of the spectral threshold to be within $\pm 0.05 \mathrm{eV}$ limit, that is, comparable to the typical IPE measurement accuracy. This conclusion finds an independent support from the observation that there is no difference in the band offsets between the $\operatorname{In}_{x} \mathrm{Ga}_{1-x} \mathrm{As}_{\mathrm{Al}} / \mathrm{Al}_{2} \mathrm{O}_{3}$ interfaces for $x=0.53$ and $x=0.75$ [137].

In turn, the photocurrent yield spectra at the (100)InAs/a$\mathrm{Al}_{2} \mathrm{O}_{3}$ interface shown in Figure 27 for three samples with different InAs doping type and surface preparation are quite reproducible and exhibit a clear feature at $h v \approx 4.5 \mathrm{eV}$, reflecting the $E_{0}^{\prime} / E_{0}^{\prime}+\Delta_{0}^{\prime}$ singularity of the InAs crystal [138]. The latter gives a clear indication that electron IPE from InAs provides the dominant contribution to the detected photocurrent. Determination of the corresponding spectral threshold(s) is illustrated in Figure 28 for the n-type InAs/a$\mathrm{Al}_{2} \mathrm{O}_{3}(20 \mathrm{~nm})$ sample revealing one spectral threshold in the low-field range which splits in two by increasing the positive voltage applied to the top metal electrode. The appearance of two IPE thresholds may be related, again, to the presence of an IL or to the incorporation of easily diffusing In into the alumina film. Since the spectral thresholds are clearly field dependent, the Schottky plots were used to determine the zero-field barrier value which corresponds to the energy offset between the VB of InAs and the CB of alumina. The Schottky plots shown in Figure 29 for GaAs, InAs, and two $\operatorname{In}_{x} \mathrm{Ga}_{1-x}$ As alloys reveal that the highest IPE thresholds associated with the direct IPE of electrons from the semiconductor into the $\mathrm{CB}$ of $\mathrm{a}-\mathrm{Al}_{2} \mathrm{O}_{3}$ are nearly the same and yield zero-field barrier value of $3.45 \pm 0.10 \mathrm{eV}$ in agreement with the previously published result $[135,136]$ as well as with a more recent evaluation [139].

By contrast, the lower thresholds show a very different behavior in InAs as compared to the GaAs and $\operatorname{In}_{x} \mathrm{Ga}_{1-x} \mathrm{As}$ interfaces. In the latter two cases the $\Phi_{e}$ (low) is clearly split by $\approx 1 \mathrm{eV}$ which corresponds to electron IPE into the IL, while at the InAs $/ \mathrm{Al}_{2} \mathrm{O}_{3}$ interface the difference between $\Phi_{e}$ (high) and $\Phi_{e}$ (low) is much smaller. Furthermore, extrapolation to zero field results in the same $\operatorname{InAs} / \mathrm{a}-\mathrm{Al}_{2} \mathrm{O}_{3}$ barrier value, suggesting perturbation of the interface barrier either by a built-in charge or by In incorporated into the alumina. The recently reported somewhat lower threshold of electron IPE at the InAs $/ \mathrm{Al}_{2} \mathrm{O}_{3}$ interface of $3.2 \mathrm{eV}$ [140] probably reflects the $\Phi_{e}$ (low) found in our samples. To conclude, the comparison between $\operatorname{In}_{x} \mathrm{Ga}_{1-x}$ As materials over the whole range of composition $0 \leq x \leq 1$ indicates that, within the measurement accuracy of $\pm 0.05 \mathrm{eV}$, the $\mathrm{VB}$ of semiconductor retains its energy position with respect to the reference level of a- $\mathrm{Al}_{2} \mathrm{O}_{3} \mathrm{CB}$ bottom. Then the bandgap shrinking from $1.42 \mathrm{eV}$ in GaAs to $0.35 \mathrm{eV}$ in InAs must occur via the semiconductor CB shift.

7.2. Interfaces of Phosphides and Antimonides of In and $G a$ with Oxide Insulators. While the IPE spectroscopy provides a clear picture of the semiconductor bandgap edges shifts when moving from GaAs to InAs by changing the group III cation, it remains unclear what trends will emerge when the group $\mathrm{V}$ anion is changed from As to $\mathrm{P}$ or to $\mathrm{Sb}$. In order to trace these 


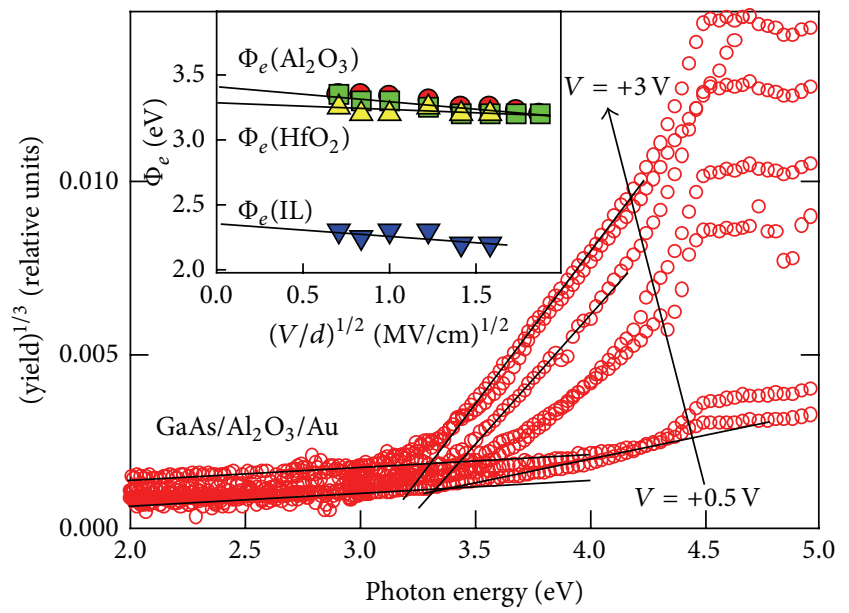

(a)

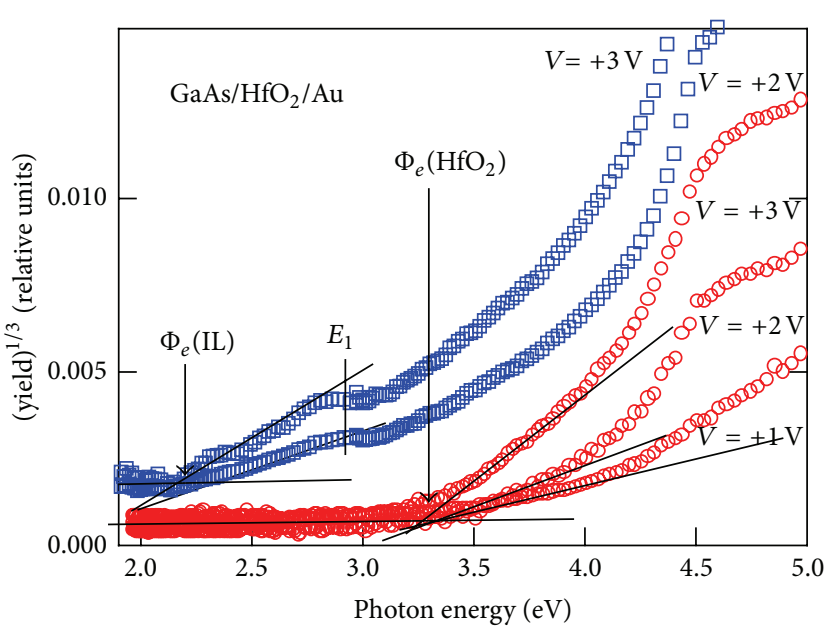

(b)

Figure 24: The $Y^{1 / 3}-h v$ plots in samples with a- $\mathrm{Al}_{2} \mathrm{O}_{3}$ (a) and $\mathrm{HfO}_{2}$ (b) insulators measured under different positive voltages applied to the top Au electrode used to determine the electron IPE spectral thresholds. The inset in panel (a) shows the Schottky plot of the thresholds of electron IPE from the GaAs VB into the CB of $\mathrm{Al}_{2} \mathrm{O}_{3}(\mathrm{O}, \square)$ and $\mathrm{HfO}_{2}(\triangle)$ and into the IL $(\nabla)$. Vertical arrows mark the observed threshold energies and lines guide the eye.

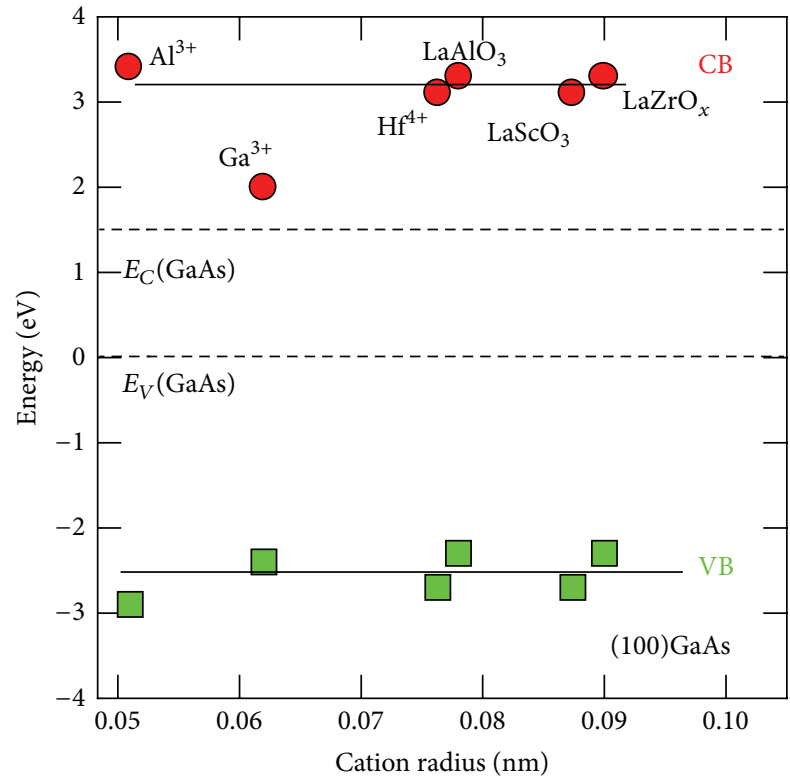

FIgURE 25: Energies of the VB top and CB bottom in different oxides as a function of cation radius referenced to the band edges for GaAs crystal as inferred from the IPE experiments. The top of the GaAs $\mathrm{VB}$ is at $\delta E_{V}=0.2 \mathrm{eV}$ below the silicon $\mathrm{VB}$ as measured relative to the common a- $\mathrm{Al}_{2} \mathrm{O}_{3} \mathrm{CB}$ bottom reference level.

trends we addressed the band alignment at the interfaces of $\mathrm{InP}, \mathrm{GaP}, \mathrm{InSb}$, and $\mathrm{GaSb}$ with a- $\mathrm{Al}_{2} \mathrm{O}_{3}$ which bandgap edges will serve, again, as the energy scale references.

The electron IPE spectra from (100)InP into a- $\mathrm{Al}_{2} \mathrm{O}_{3}$ shown in Figure 30 for samples with different pre-deposition treatment of the semiconductor surface can be correlated with the electron microscopy results suggesting, again,

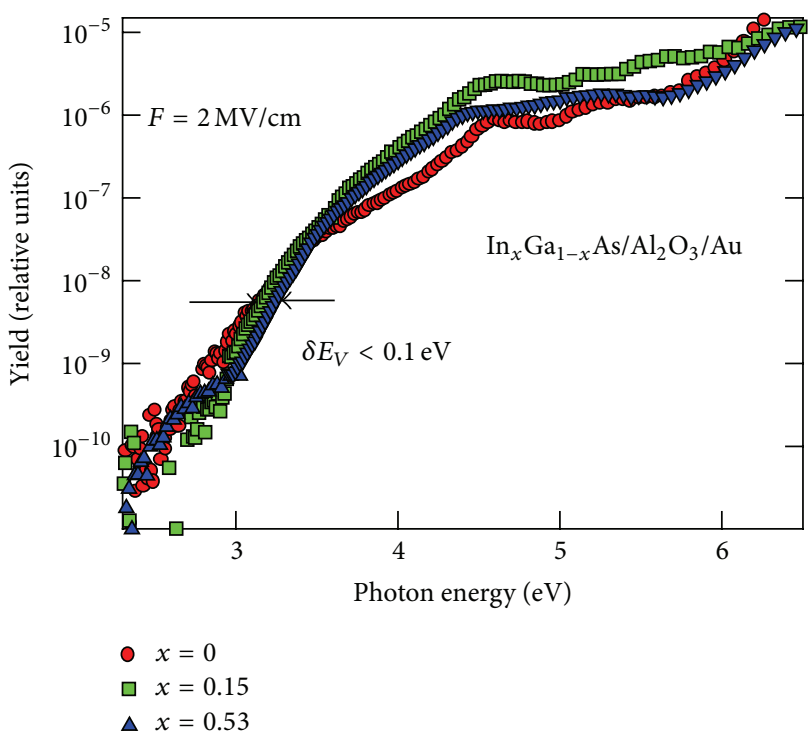

FIGURE 26: Semilogarithmic plots of quantum yield spectral dependences in samples with $\mathrm{Al}_{2} \mathrm{O}_{3}$ insulator on $\mathrm{In}_{x} \mathrm{Ga}_{1-x}$ As epitaxial layers for different concentrations of In in the semiconductor. The curves are measured under positive bias on the top metal electrode corresponding to the average strength of electric field in the oxide of $2 \mathrm{MV} / \mathrm{cm}$.

a combination of the direct IPE into the $\mathrm{CB}$ of $\mathrm{a}-\mathrm{Al}_{2} \mathrm{O}_{3}$ and of the transitions mediated by the IL grown by oxidation of InP during ALD of alumina [141]. These two photoinjection paths give rise to two different spectral thresholds which can be assigned according to their sensitivity to the IL thickness. The IL-insensitive threshold (cf. Figure 4(a) in [141]) corresponds to the direct IPE process. Being extrapolated to zero strength of the electric field in the insulating stack, it yields the barrier 


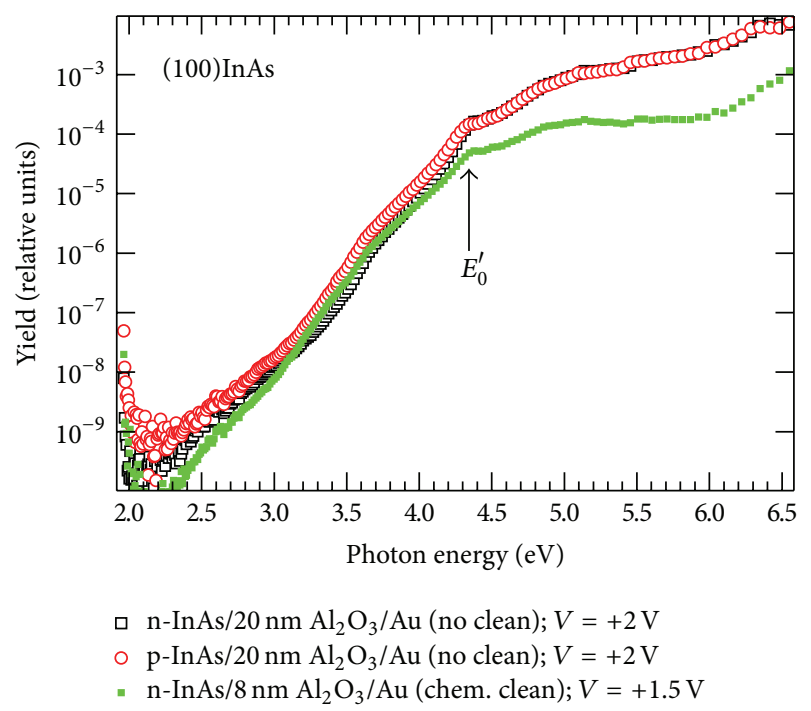

FIgURE 27: Semilogarithmic plot of the IPE quantum yield as a function of photon energy as measured on (100) InAs/a- $\mathrm{Al}_{2} \mathrm{O}_{3} / \mathrm{Au}$ samples prepared by using different alumina growth schemes. The vertical arrow $E_{0}^{\prime}$ marks the energy of direct optical transitions in the InAs crystal.
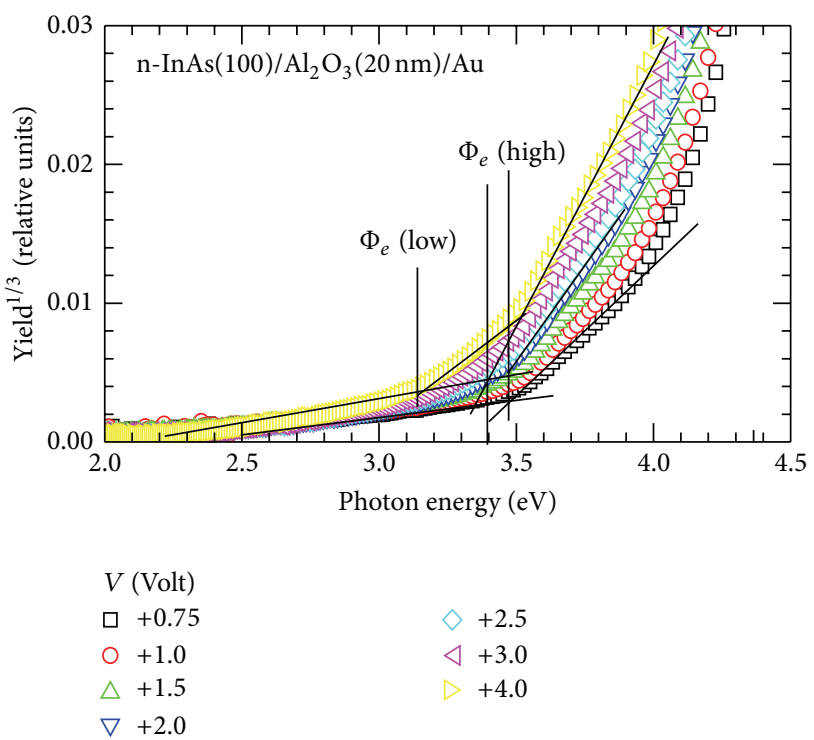

FIGURE 28: Cube root of the IPE yield as a function of photon energy as measured at different bias applied to the (100) InAs/a- $\mathrm{Al}_{2} \mathrm{O}_{3}$ $(20 \mathrm{~nm}) / \mathrm{Au}$ capacitor which are used to determine the spectral thresholds. The inferred thresholds $\Phi_{e}$ (low/high) of electron IPE from the $\mathrm{VB}$ of InAs into the $\mathrm{CB}$ of $\mathrm{a}-\mathrm{Al}_{2} \mathrm{O}_{3}$ are indicated by vertical lines. Lines guide the eye.

of $4.05 \pm 0.10 \mathrm{eV}$ between the top of the InP VB and the bottom of the a- $\mathrm{Al}_{2} \mathrm{O}_{3} \mathrm{CB}$. This result importantly indicates that variation of the group $\mathrm{V}$ element in $\mathrm{A}_{\mathrm{III}} \mathrm{B}_{\mathrm{V}}$ semiconductors has a profound effect on the VB energy as opposed to the change of the group III element.

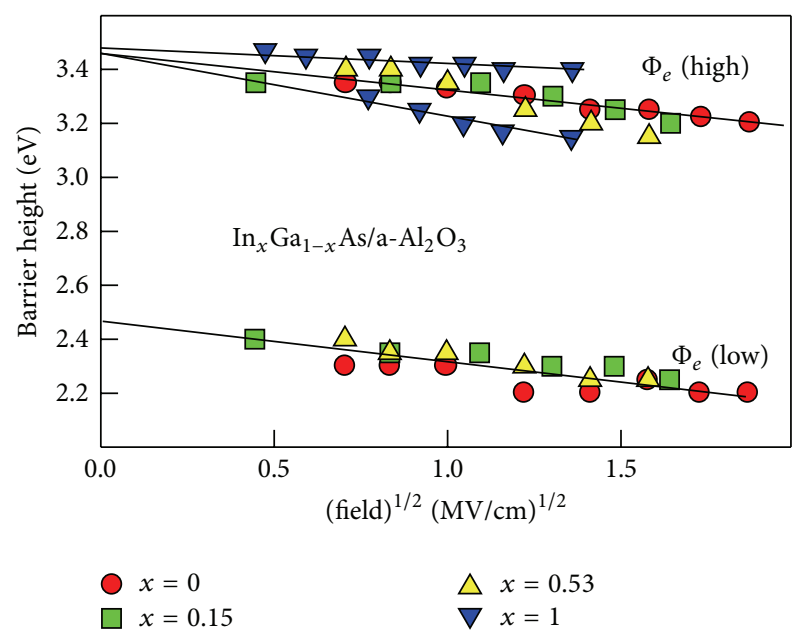

FIGURE 29: Schottky plots of the electron IPE spectral thresholds observed in samples with $\mathrm{Al}_{2} \mathrm{O}_{3}$ insulator and different content $x$ of In in the semiconductor substrate. The "high" spectral threshold corresponds to the energy barrier between the top of the $\operatorname{In}_{x} \mathrm{Ga}_{1-x} \mathrm{As}$ $\mathrm{VB}$ and the bottom of the deposited oxide CB. The "low" barriers stem from the electron IPE mediated by the narrow-gap $\operatorname{In}_{x} \mathrm{Ga}_{1-x}$ As "native" oxide present at the interface. Lines guide the eye.

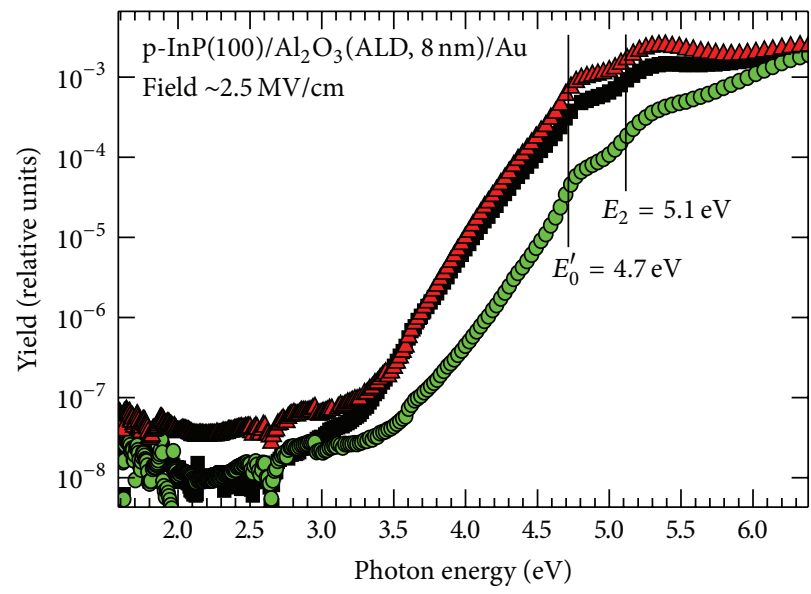
Surface treatment
- $\mathrm{HCl}$
$\mathrm{O} \mathrm{UVO}_{3}$
$\triangle\left(\mathrm{NH}_{4}\right)_{2} \mathrm{~S}$

FIGURE 30: Semilogarithmic plot of the IPE quantum yield as a function of photon energy as measured on p-type (100)InP/a$\mathrm{Al}_{2} \mathrm{O}_{3}(8 \mathrm{~nm}) / \mathrm{Au}$ samples prepared by using different InP surface treatments prior to the alumina growth by ALD. The vertical arrows $E_{0}$ and $E_{2}$ mark the energy of direct optical transitions in the InP crystal.

The same trend can be inferred from Figure 31 which shows photocurrent yield spectra measured on the n$\mathrm{GaP}(100) / \mathrm{a}-\mathrm{Al}_{2} \mathrm{O}_{3}(20 \mathrm{~nm}) / \mathrm{Al}$ sample under different bias applied to the metal electrode. Though the low-field measurements are distorted by the photocurrent related to recharging traps in the wide-gap semiconductor substrate 


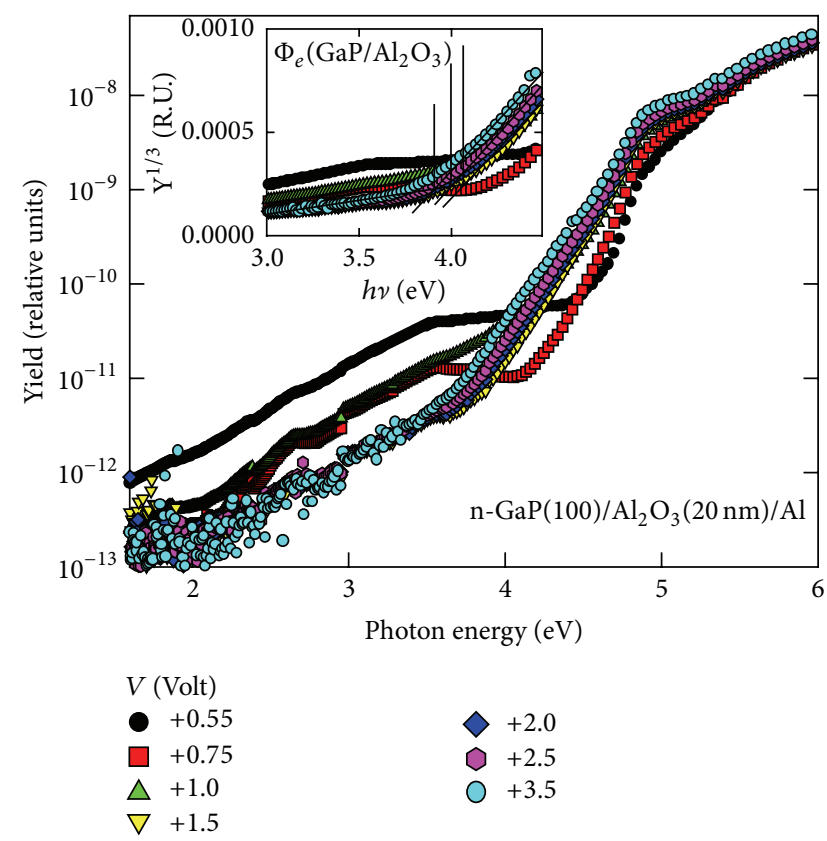

FIGURE 31: Semilogarithmic plot of the IPE quantum yield as a function of photon energy as measured on (100) GaP/a- $\mathrm{Al}_{2} \mathrm{O}_{3}(20 \mathrm{~nm}) / \mathrm{Al}$ sample with the applied bias varying from $0.55 \mathrm{~V}$ to $3.5 \mathrm{~V}$. The inset illustrates determination of electron IPE spectral threshold $\Phi_{e}$ using $Y^{1 / 3}-h v$ plots. Lines guide the eye.

$\left(E_{g}(\mathrm{GaP})=2.24 \mathrm{eV}\right.$ at $\left.300 \mathrm{~K},[142]\right)$, upon applying a bias exceeding $+1 \mathrm{~V}$, one observes the yield increase above $h v=$ $4 \mathrm{eV}$ by nearly three orders of magnitude. The corresponding spectral curves exhibit characteristic structure near $h v=$ $5 \mathrm{eV}$ which reflects excitation of optical transitions $\Gamma_{15 \mathrm{v}}-\Gamma_{15 \mathrm{c}}$ and $\mathrm{X}_{5 \mathrm{v}}-\mathrm{X}_{1 \mathrm{c}}$ between high symmetry points in the Brillouin zone of the GaP substrate crystal [143]. The latter leaves no doubt that the signal with a spectral onset close to $4 \mathrm{eV}$ originates from the IPE of electrons from the VB of GaP into the $\mathrm{CB}$ of alumina. The corresponding field-dependent spectral thresholds are inferred from the $Y^{1 / 3}-h \nu$ plots as schematically shown in the inset and, upon extrapolation to zero field using the Schottky plot (not shown), yield the barrier height between the top of the GaP VB and the bottom of the a- $\mathrm{Al}_{2} \mathrm{O}_{3} \mathrm{CB}$ of $4.1 \pm 0.1 \mathrm{eV}$. The latter value coincides with the InP/a- $\mathrm{Al}_{2} \mathrm{O}_{3}$ barrier height within the experimental error margin importantly indicating that the VB top energy in $\mathrm{A}_{\mathrm{III}} \mathrm{B}_{\mathrm{V}}$ compounds is determined by the type of the group $\mathrm{V}$ anion. Therefore, the bandgap widening from $1.35 \mathrm{eV}$ in InP to $2.24 \mathrm{eV}$ in GaP occurs, again, through the shift of the semiconductor $\mathrm{CB}$ bottom edge.

More evidence is provided to the above conclusions by the analysis of band offsets at the (100) $\mathrm{InSb} / \mathrm{a}-\mathrm{Al}_{2} \mathrm{O}_{3}$ [137] and $(100) \mathrm{GaSb} / \mathrm{a}-\mathrm{Al}_{2} \mathrm{O}_{3}$ interfaces $[144,145]$. From the overview IPE spectra shown in Figures 32 and 33, respectively, it is evident that the electron IPE from these semiconductors has an energy onset close to $3.0 \mathrm{eV}$. More elaborate analysis $[144,146]$ yields the same values of the energy barrier values of $3.05 \pm 0.10 \mathrm{eV}$ between the top of the VBs in InSb and $\mathrm{GaSb}$ and the bottom of the $\mathrm{Al}_{2} \mathrm{O}_{3} \mathrm{CB}$, indicating that in
TABLE 1: Bandgap width of several semiconductors $\left(E_{g}\right)$, the electron photoemission barrier $\left(\Phi_{e}\right)$, and the corresponding $\mathrm{CB}$ and $\mathrm{VB}$ offsets $\Delta E_{C}$ and $\Delta E_{V}$ at the interfaces with a- $\mathrm{Al}_{2} \mathrm{O}_{3}\left(E_{g}=6.1 \mathrm{eV}\right)$. All energies are in $\mathrm{eV}( \pm 0.1 \mathrm{eV})$.

\begin{tabular}{lcccc}
\hline $\mathrm{sc}$ & $E_{g}(300 \mathrm{~K})$ & $\Phi_{e}$ & $\Delta E_{C}$ & $\Delta E_{V}$ \\
\hline $\mathrm{Si}$ & 1.12 & 3.25 & 2.1 & 2.9 \\
$\mathrm{Ge}$ & 0.67 & 2.85 & 2.2 & 3.3 \\
$\mathrm{GaAs}$ & 1.42 & 3.45 & 2.0 & 2.7 \\
$\mathrm{In}_{0.53} \mathrm{Ga}_{0.47} \mathrm{As}$ & 0.74 & 3.45 & 2.7 & 2.7 \\
$\mathrm{InAs}$ & 0.35 & 3.45 & 3.1 & 2.7 \\
$\mathrm{GaP}$ & 2.24 & 4.1 & 1.8 & 2.0 \\
$\mathrm{InP}$ & 1.35 & 4.05 & 2.7 & 2.05 \\
$\mathrm{GaSb}$ & 0.73 & 3.05 & 2.3 & 3.05 \\
$\mathrm{InSb}$ & 0.17 & 3.05 & 2.9 & 3.05 \\
\hline
\end{tabular}

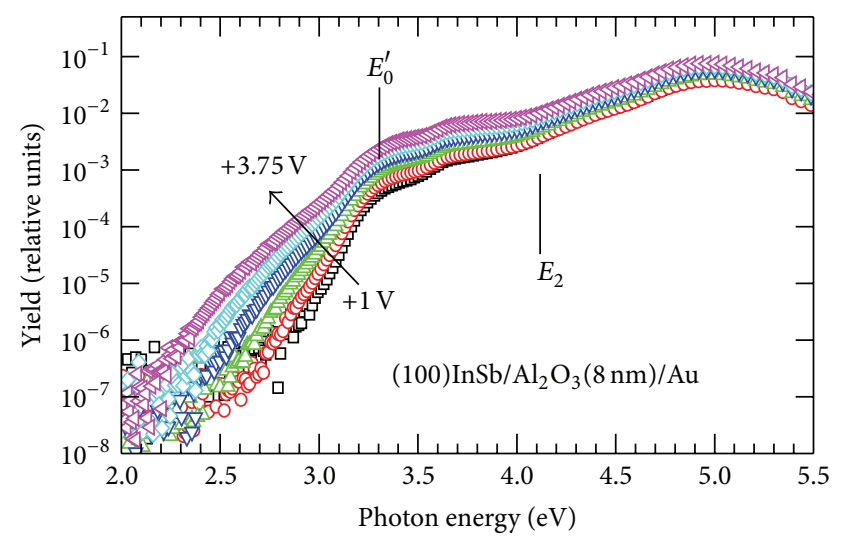

FIGURE 32: Semilogarithmic plot of the IPE quantum yield as a function of photon energy as measured on (100) InSb/a- $\mathrm{Al}_{2} \mathrm{O}_{3}(8 \mathrm{~nm}) / \mathrm{Au}$ sample with the applied bias varying from $1 \mathrm{~V}$ to $3.75 \mathrm{~V}$. The vertical arrows $E_{0}$ and $E_{2}$ mark the energy of direct optical transitions in the InSb crystal.

both antimonides the top of the $\mathrm{VB}$ is shifted by $\approx 0.4 \mathrm{eV}$ with respect to arsenides and by $\approx 1 \mathrm{eV}$ as compared to phosphides of the same metals. In order to illustrate this behavior using experimental data, the electron IPE spectra from different semiconductor crystals into the same a- $\mathrm{Al}_{2} \mathrm{O}_{3}$ insulator are compared in Figure 34. Though there are differences in the range of low photon energies in the cases of $\mathrm{GaSb}$ and $\mathrm{GaP}$ associated with the use of $\mathrm{p}^{+}$-doped substrates [145] and with the low $\mathrm{CB}$ offset, respectively, the general trend of the VB shift following the change of the group $\mathrm{V}$ anion is clearly seen. At the same time, while keeping the group $\mathrm{V}$ component unchanged, variation in the group III cation sort causes no measurable variations in the VB top energy leaving the shift of the $\mathrm{CB}$ bottom edge entirely responsible for the bandgap variations. To provide the reader with the relevant numerical data, in Table 1 are listed the accepted values of semiconductor bandgap widths at $300 \mathrm{~K}$, the electron IPE barriers measured by IPE at the interfaces of the corresponding semiconductors with a- $\mathrm{Al}_{2} \mathrm{O}_{3}$, and the $\mathrm{CB}$ and $\mathrm{VB}$ offsets calculated using the listed semiconductor bandgaps and the a- $\mathrm{Al}_{2} \mathrm{O}_{3}$ bandgap value of $6.1 \mathrm{eV}$, respectively. 


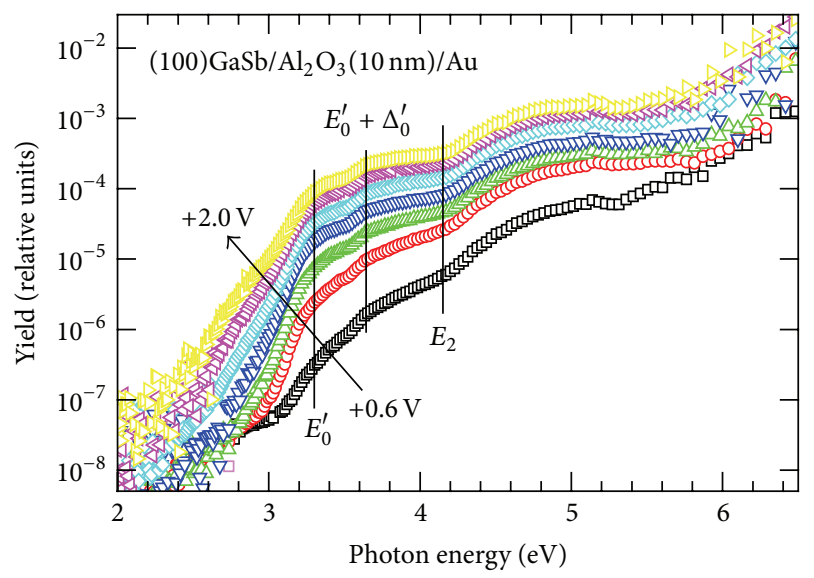

FIGURE 33: Semilogarithmic plot of the IPE quantum yield as a function of photon energy as measured on (100) GaSb/a$\mathrm{Al}_{2} \mathrm{O}_{3}(10 \mathrm{~nm}) / \mathrm{Au}$ sample with the applied bias varying from $0.6 \mathrm{~V}$ to $2.0 \mathrm{~V}$. The vertical arrows $E_{0}, E_{0}^{\prime}+\Delta_{0}^{\prime}$, and $E_{2}$ mark the energy of direct optical transitions in the GaSb crystal.

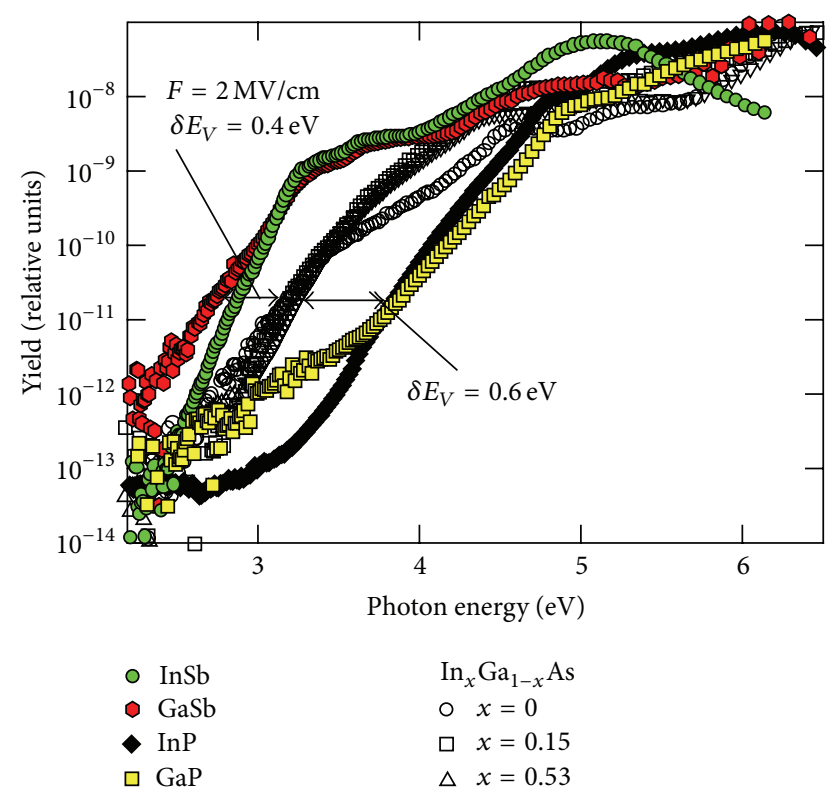

Figure 34: Comparison of the yield spectra for (100) faces of GaSb, GaAs, GaP, $\operatorname{In}_{x} \mathrm{Ga}_{1-x}$ As $(x=0.15,0.53) \mathrm{InSb}$, and $\mathrm{InP}$ in contact with a $10-20 \mathrm{~nm}$ thick a- $\mathrm{Al}_{2} \mathrm{O}_{3}$ insulating film measured under approximately equal strength of electric field in the oxide (positive bias applied to the opposite metal electrode). The inferred variations in the semiconductor VB top energies $\left(\delta E_{V}\right)$ measured relative to the common reference level of $\mathrm{Al}_{2} \mathrm{O}_{3} \mathrm{CB}$ edge are indicated by arrows.

7.3. IPE from Semiconductor Heterojunctions and Band Offset Transitivity. The results presented so far pertain to interfaces of bulk or bulk-like layers of semiconductors aiming at revealing behavior of the intrinsic DOS at the interfaces with insulating oxides. In recent years, however, a large number of semiconductor heterojunctions have been introduced at the interfaces with insulating oxides to facilitate better interface passivation $[147,148]$, to introduce additional barrier layer

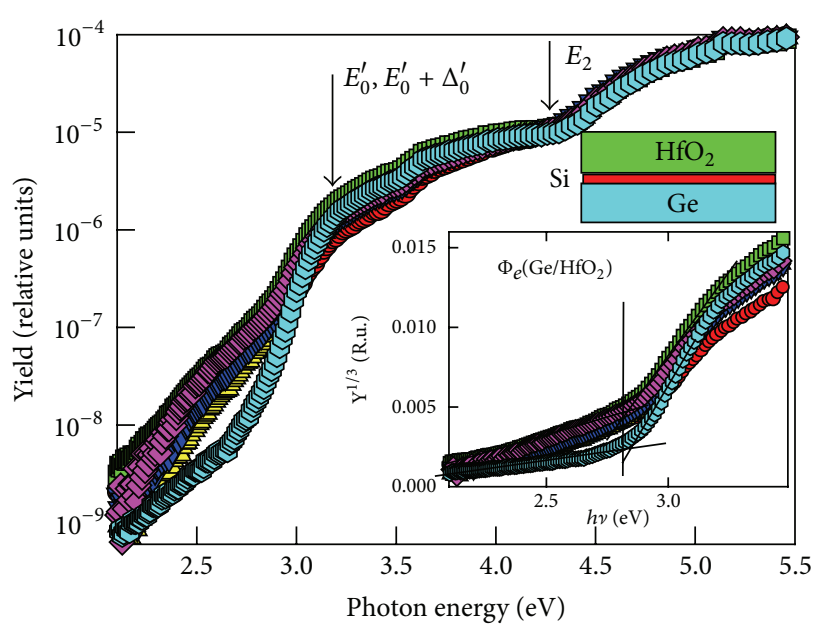

[Si] MLs

$\begin{array}{ll}\bigcirc 0 & \triangle 4 \\ 02 & \nabla 5 \\ \square 3 & \diamond 6\end{array}$

FIGURE 35: Semiogarithmic spectral plots of the electron IPE yield as measured in $(100) \mathrm{Ge} / \mathrm{Si} / \mathrm{HfO}_{2} / \mathrm{Au}$ samples under $+1.5 \mathrm{~V}$ bias on the $\mathrm{Au}$ electrode for different thicknesses (in monolayers of $\mathrm{Si}$ ) of the inserted Si IL. The vertical lines mark the energy onsets of direct optical transitions between high symmetry points in the Brillouin zone of the Ge substrate crystal. The inset illustrates determination of the electron IPE threshold $\Phi_{e}\left(\mathrm{Ge} / \mathrm{HfO}_{2}\right)$ marked by the vertical line using $Y^{1 / 3}-h v$ plots. Lines guide the eye.

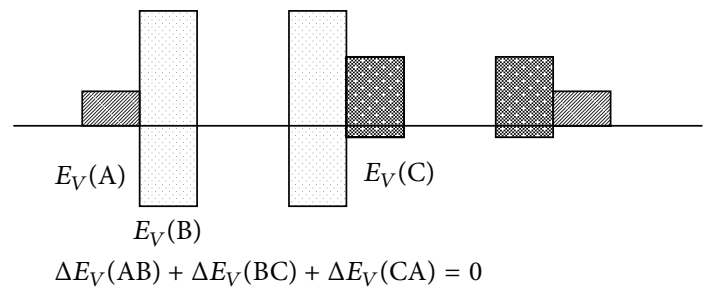

FIGURE 36: Schematic representation of the valence band offset $\Delta E_{V}$ transitivity principle in semiconductor heterojunctions.

[149], to fabricate quantum well channel structure [150] or a tunneling transistors [151]. In all these cases the band offset in the semiconductor heterojunction itself plays a crucial role in attaining the desired functionality of these devices. Since the thicknesses of the involved semiconductor layers are usually in the range of a few nanometers, that is, it is comparable to the mean photoelectron escape length, it becomes in principle possible to observe IPE from two component materials of a heterojunction. Then, by comparing the energy thresholds of electron IPE from their VBs into the $\mathrm{CB}$ of the same insulator, one can directly measure the band offset value in the heterojunction itself $[139,140]$. The fundamental difficulty of this approach consists in the influence of the built-in electric field in the heterojunction. This field is usually related to the presence of ionized dopants and may shift the energy 
of the band edges across the heterojunction region which gives rise to the IPE signal. As a result, two problems emerge at once. First, there is no uniquely defined semiconductor band edge energy since it is affected by the electrostatic shifts. Second, the application of the Schottky description to the image forces becomes questionable since the image interactions arise at each interface between materials with different dielectric permittivity [89-91].

Here we would like to consider an alternative approach to the evaluation of band offsets in semiconductor heterojunctions based on the earlier reported experimental results indicating the absence of substantial structure- or composition-sensitive contributions to the barriers at the interfaces of semiconductors with insulating oxides [116]. In this work the IPE barrier measured between the VB of a semiconductor crystal and the oxide CB bottom was analyzed as a function of thickness of a thin semiconductor layer of a dissimilar semiconductor inserted between the substrate and the insulating oxide. The typical result is illustrated in Figure 35 in which electron IPE spectra from the VB of germanium into the $\mathrm{CB}$ of $\mathrm{HfO}_{2}$ are shown as affected by the thickness of Si IL (from 2 to $6 \mathrm{MLs}$ ) epitaxially grown on $\mathrm{Ge}$ prior to $\mathrm{HfO}_{2}$ deposition. The same optical singularities (labeled according to Adachi, [152]) observed in the spectra leave no doubt that in all cases electron IPE from $\mathrm{Ge}$ is dominating the photocurrent. The spectral thresholds inferred from the standard $Y^{1 / 3}-h v$ plots and shown in the inset reveal that the electron IPE threshold $\Phi_{e}\left(\mathrm{Ge} / \mathrm{HfO}_{2}\right)$ is insensitive to the presence of the Si IL or to its thickness. In other words, the additional semiconductor IL does not affect the energy of the bandgap edges in the substrate crystal. This result is in general agreement with the earlier indicated negligible effect of the ILs on the interface band alignment. The evidence for the validity of this conclusion in the case of interfaces of $\mathrm{A}_{\mathrm{III}} \mathrm{B}_{\mathrm{V}}$ semiconductors is also presented in [116].

This experimental observation provides strong support to the hypothesis that the so-called band offset transitivity rule is valid at the interfaces between semiconductors and oxide insulators. According to this rule [153], the band offsets observed at the interfaces of three semiconductors A, B, and C satisfy the condition

$$
\Delta E_{V}(\mathrm{AB})+\Delta E_{V}(\mathrm{BC})+\Delta E_{V}(\mathrm{CA})=0,
$$

which is schematically illustrated in Figure 36 in which material $\mathrm{B}$ is taken as the wide-gap insulator, for example, $\mathrm{a}-\mathrm{Al}_{2} \mathrm{O}_{3}$ used in the present experimental analysis. The experimentally observed validity of the transitivity rule indicates that the $\mathrm{VB}$ offset of interest $\left[\Delta E_{V}(\mathrm{CA})\right]$ can directly be evaluated if the offsets (or IPE barriers) at the interfaces $\mathrm{AB}$ and $\mathrm{BC}$ are known.

This concept allows for determination of the intrinsic band lineup unaffected by the influence of electric fields or charged impurities, and therefore, provides a reliable firstorder evaluation of the heterojunction band offsets. In this way the complete band edge profile at the interfaces of semiconductor heterojenctons with insulating oxides can be found. Furthermore, thanks to the revealed sensitivity of the $\mathrm{VB}$ energies in $\mathrm{A}_{\mathrm{III}} \mathrm{B}_{\mathrm{V}}$ semiconductors exclusively to the sort of group V element, this analysis can be extended to the alloys with variable In or Ga composition by using the semiconductor bandgap values known from optical measurements.

\section{Conclusions}

The spectral analysis of the IPE photocurrents is shown to be capable of delivering a vast amount of information regarding electron energy band alignment at interfaces of semiconductors with insulator materials provided the nonIPE related components are identified and eliminated. Using the IPE/PC spectroscopy, several general trends in the evolution of electron band offsets have been revealed. First, with the bulk electron DOS determining the semiconductor/oxide interface band alignments, the interface barriers are expected to be mostly sensitive to the bandgap width and electron affinity of the materials in contact. Second, on the oxide side of the interface, the energy of the O2p-derived VB top can be used as the convenient reference. It is found to be nearly invariant (within $\pm 0.2 \mathrm{eV}$ limit) in the oxides with large $\left(r_{i} \gtrsim 0.07 \mathrm{~nm}\right)$ cations as well as in amorphous oxides of even smaller cations $\left(r_{i}>0.05 \mathrm{~nm}\right)$. As a result, the band offsets can be estimated on the basis of the known oxide bandgap and the energies of semiconductor bandgap edges known from the IPE experiments. The bandgap widening in the oxides with compact $\left(r_{i}<0.07 \mathrm{~nm}\right)$ cations upon crystallization appears to be predominantly caused by the downshift of the $\mathrm{O} 2 \mathrm{p}$-derived VB states. Third, on the semiconductor side of the interface significant differences are revealed between the composition-sensitive bandgap shifts in $\mathrm{A}_{\mathrm{IV}} \mathrm{B}_{\mathrm{IV}}$ and $\mathrm{A}_{\mathrm{III}} \mathrm{B}_{\mathrm{V}}$ materials. In the latter, case the $\mathrm{VB}$ top energy appears to be insensitive to the type of cation (In or $\mathrm{Ga}$ ), while varying dramatically if the group $\mathrm{V}$ anion is changed. Nevertheless, for both classes of semiconductors the IPE results suggest the validity of the band offset transitivity rule which allows for an easy evaluation of band offsets not only at the semiconductor/oxide interfaces but also in semiconductor heterojunctions.

\section{Conflict of Interests}

The author declares that there is no conflict of interests regarding the publication of this paper.

\section{Acknowledgments}

The author would like to thank a large group of colleagues who over many years have provided invaluable contribution to the experiments overviewed in this work: A. Stesmans, M. Houssa, M. Badylevich, W.-C. Wang, H.-Y. Chou, S. Shamuilia, F. De Stefano, Ch. Adelmann, G. Brammertz, S. DeGendt, A. Delabie, L. Goux, M. Heyns, D. Lin, C. Merckling, M. Meuris, L. Pantisano, S. Sionke, L. Souriau, W. Tsai, B. Vincent, C. Zhao, J. Schubert, A. Laha., H.-J. Osten, A. Fissel, P. K. Hurley, E. O'Connor, K. Cherkaoui, M. Pemble, I. Povey, S. B. Newcomb, A. Molle, L. Lamagna, G. Mavrou, A. Dimoulas, L. Edge, D. G. Schlom, S. A. Campbell, P. D. Ye, M. Passlack, and R. Droopad. The author acknowledges partial 
support from the EU FP7 Project MORDRED (Grant no. 261868) and from the Fonds Wetenschappelijk OnderzoekVlaanderen (Project G.0C05.13).

\section{References}

[1] G. D. Wilk, R. M. Wallace, and J. M. Anthony, "High- $\kappa$ gate dielectrics: current status and materials properties considerations," Journal of Applied Physics, vol. 89, no. 10, pp. 5243-5275, 2001.

[2] M. Houssa, Ed., High-K Gate Dielectrics, IoP Publishing, Bristol, UK, 2004.

[3] H. R. Huff and D. C. Gilmer, Eds., High Dielectric Constant Materials, Springer, Berlin, Germany, 2005.

[4] A. Dimoulas, E. Gusev, P. C. McIntyre, and M. Heyns, Eds., Advanced Gate Stacks for High-Mobility Semiconductors, Springer, Berlin, Germany, 2007.

[5] G. He and Z. Sun, Eds., High-K Gate Dielectrics for C-MOS Technology, Wiley-VCH, Weinheim, Germany, 2012.

[6] J. Robertson, "Band offsets of wide-band-gap oxides and implications for future electronic devices," Journal of Vacuum Science and Technology B, vol. 18, no. 3, pp. 1785-1791, 2000.

[7] D. G. Schlom and J. H. Haeni, "A thermodynamic approach to selecting alternative gate dielectrics," MRS Bulletin, vol. 27, no. 3, pp. 198-204, 2002.

[8] J. Robertson, "High dielectric constant gate oxides for metal oxide Si transistors," Reports on Progress in Physics, vol. 69, no. 2, pp. 327-396, 2006.

[9] D. G. Schlom, S. Guha, and S. Datta, "Gate oxides beyond $\mathrm{SiO}_{2}$," MRS Bulletin, vol. 33, no. 11, pp. 1017-1025, 2008.

[10] J. A. Kittl, K. Opsomer, M. Popovici et al., "High-k dielectrics for future generation memory devices (Invited Paper)," Microelectronic Engineering, vol. 86, no. 7-9, pp. 1789-1795, 2009.

[11] V. V. Afanas'ev, M. Houssa, A. Stesmans, G. J. Adriaenssens, and M. M. Heyns, "Band alignment at the interfaces of $\mathrm{Al}_{2} \mathrm{O}_{3}$ and $\mathrm{ZrO}_{2}$-based insulators with metals and $\mathrm{Si}$," Journal of NonCrystalline Solids, vol. 303, no. 1, pp. 69-77, 2002.

[12] J. Robertson, "Band offsets, Schottky barrier heights, and their effects on electronic devices," Journal of Vacuum Science and Technology A, vol. 31, Article ID 050821, 2013.

[13] W. M. Lau, "Use of surface charging in X-ray photoelectron spectroscopic studies of ultrathin dielectric films on semiconductors," Applied Physics Letters, vol. 54, no. 4, pp. 338-340, 1989.

[14] W. M. Lau, "Effects of a depth-dependent specimen potential on X-ray photoelectron spectroscopic data," Journal of Applied Physics, vol. 65, no. 5, pp. 2047-2052, 1989.

[15] J. L. Alay and M. Hirose, "The valence band alignment at ultrathin $\mathrm{SiO}_{2} / \mathrm{Si}$ interfaces," Journal of Applied Physics, vol. 81, no. 3, pp. 1606-1608, 1997.

[16] S. Toyoda, J. Okabayashi, H. Kumigashira et al., "Precise determination of band offsets and chemical states in SiN/Si studied by photoemission spectroscopy and X-ray absorption spectroscopy," Applied Physics Letters, vol. 87, no. 10, Article ID 102901, 2005.

[17] T. Tanimura, S. Toyoda, H. Kamada et al., "Photoinduced charge-trapping phenomena in metal/high-k gate stack structures studied by synchrotron radiation photoemission spectroscopy," Applied Physics Letters, vol. 96, no. 16, Article ID 162902, 2010.
[18] H. Nohira, W. Tsai, W. Besling et al., "Characterization of ALCVD- $\mathrm{Al}_{2} \mathrm{O}_{3}$ and $\mathrm{ZrO}_{2}$ layer using X-ray photoelectron spectroscopy," Journal of Non-Crystalline Solids, vol. 303, no. 1, pp. 83-87, 2002.

[19] M. Perego, G. Seguini, and M. Fanciulli, "XPS and IPE analysis of $\mathrm{HfO}_{2}$ band alignment with high-mobility semiconductors," Materials Science in Semiconductor Processing, vol. 11, no. 5, pp. 221-225, 2008.

[20] E. Bersch, M. Di, S. Consiglio, R. D. Clark, G. J. Leusink, and A. C. Diebold, "Complete band offset characterization of the $\mathrm{HfO}_{2} / \mathrm{SiO}_{2} / \mathrm{Si}$ stack using charge corrected X-ray photoelectron spectroscopy," Journal of Applied Physics, vol. 107, no. 4, Article ID 043702, 2010.

[21] V. V. Afanas'ev and A. Stesmans, "Internal photoemission at interfaces of high-k insulators with semiconductors and metals," Journal of Applied Physics, vol. 102, Article ID 081301, 2007.

[22] V. V. Afanas'ev, Internal Photoemission Spectroscopy: Principles and Applications, Elsevier, Oxford, UK, 2008.

[23] V. V. Afanas'ev, M. Badylevich, A. Stesmans, A. Laha, H. J. Osten, and A. Fissel, "Size-dependent interface band alignment between $\mathrm{Si}$ nanocrystals and lattice-matched $\mathrm{Gd}_{2} \mathrm{O}_{3}$," Applied Physics Letters, vol. 95, no. 10, Article ID 102107, 2009.

[24] G. Seguini, S. Schamm-Chardon, P. Pellegrino, and M. Perego, "The energy band alignment of Si nanocrystals in $\mathrm{SiO}_{2}$," Applied Physics Letters, vol. 99, no. 8, Article ID 082107, 2011.

[25] G. Seguini, C. Castro, S. Schamm-Chardon, G. BenAssayag, P. Pellegrino, and M. Perego, "Scaling size of the interplay between quantum confinement and surface related effects in nanostructured silicon," Applied Physics Letters, vol. 103, Article ID 023103, 2013.

[26] E. A. Kraut, "Heterojunction band off-sets-variation with ionization potential compared to experiment," Journal of Vacuum Science and Technology B, vol. 2, no. 3, pp. 486-490, 1984.

[27] G. R. Waldrop, G. W. Grant, S. P. Kowalszyk, and E. A. Kraut, "Measurement of semiconductor heterojunction band discontinuities by X-ray photoemission spectroscopy," Journal of Vacuum Science and Technology B, vol. 3, p. 835, 1985.

[28] M. Perego and G. Seguini, "Charging phenomena in dielectric/semiconductor heterostructures during X-ray photoelectron spectroscopy measurements," Journal of Applied Physics, vol. 110, no. 5, Article ID 053711, 2011.

[29] Z. Q. Liu, W. K. Chim, S. Y. Chiam, J. S. Pan, and C. M. Ng, "Evaluating the use of electronegativity in band alignment models through the experimental slope parameter of lanthanum aluminate heterostructures," Journal of Applied Physics, vol. 110, no. 9, Article ID 093701, 2011.

[30] X. Wang, J. Xiang, W. Wang et al., "Reexamination of band offset transitivity employing oxide heterojunctions," Applied Physics Letters, vol. 102, Article ID 031605, 2013.

[31] V. K. Adamchuk and V. V. Afanas'ev, "Internal photoemission spectroscopy of semiconductor-insulator interfaces," Progress in Surface Science, vol. 41, no. 2, pp. 111-211, 1992.

[32] U. Bangert, A. J. Harvey, and R. Keyse, "Assessment of electron energy-loss spectroscopy below $5 \mathrm{eV}$ in semiconductor materials in a VG STEM," Ultramicroscopy, vol. 68, no. 3, pp. 173-180, 1997.

[33] B. Rafferty and L. M. Brown, "Direct and indirect transitions in the region of the band gap using electron-energy-loss spectroscopy," Physical Review B, vol. 58, no. 16, pp. 10326-10337, 1998. 
[34] B. Rafferty, S. J. Pennycook, and L. M. Brown, "Zero loss peak deconvolution for bandgap EEL spectra," Journal of Electron Microscopy, vol. 49, no. 4, pp. 517-524, 2000.

[35] M. C. Cheynet, S. Pokrant, F. D. Tichelaar, and J.-L. Rouvire, "Crystal structure and band gap determination of $\mathrm{HfO}_{2}$ thin films," Journal of Applied Physics, vol. 101, no. 5, Article ID 054101, 2007.

[36] J. Park, S. Heo, J.-G. Chung et al., "Bandgap measurement of thin dielectric films using monochromated STEM-EELS," Ultramicroscopy, vol. 109, no. 9, pp. 1183-1188, 2009.

[37] S. Miyazaki, "Photoemission study of energy-band alignments and gap-state density distributions for high-k gate dielectrics," Journal of Vacuum Science and Technology B, vol. 19, no. 6, pp. 2212-2216, 2001.

[38] V. V. Afanas'ev, M. Houssa, A. Stesmans, and M. M. Heyns, "Band alignments in metal-oxide-silicon structures with atomic-layer deposited $\mathrm{Al}_{2} \mathrm{O}_{3}$ and $\mathrm{ZrO}_{2}$," Journal of Applied Physics, vol. 91, no. 5, pp. 3079-3084, 2002.

[39] S. Shamuilia, V. V. Afanas'ev, A. Stesmans et al., "Photoconductivity of Hf-based binary metal oxide systems," Journal of Applied Physics, vol. 104, no. 11, Article ID 114103, 2008.

[40] E. O. Kane, "Theory of photoelectric emission from semiconductors," Physical Review, vol. 127, no. 1, pp. 131-141, 1962.

[41] R. Y. Koyama and N. V. Smith, "Photoemission properties of simple metals," Physical Review B, vol. 2, no. 8, pp. 3049-3059, 1970.

[42] J. M. Ballantyne, "Effect of phonon energy loss on photoemissive yield near threshold," Physical Review B, vol. 6, no. 4, pp. 1436-1455, 1972.

[43] R. J. Powell, "Interface barrier energy determination from voltage dependence of photoinjected currents," Journal of Applied Physics, vol. 41, no. 6, pp. 2424-2432, 1970.

[44] R. H. Fowler, "The analysis of photoelectric sensitivity curves for clean metals at various temperatures," Physical Review, vol. 38, no. 1, pp. 45-56, 1931.

[45] A. M. Goodman, "Photoemission of electrons from n-type degenerate silicon into silicon dioxide," Physical Review, vol. 152, no. 2, pp. 785-787, 1966.

[46] C. Sebenne, D. Bolmont, G. Guichar, and M. Balkanski, "Surface states from photoemission threshold measurements on a clean, cleaved, Si (111) surface," Physical Review B, vol. 12, no. 8, pp. 3280-3285, 1975.

[47] J. E. Rowe and H. Ibach, "Surface and bulk contributions to ultraviolet photoemission spectra of silicon," Physical Review Letters, vol. 32, no. 8, pp. 421-424, 1974.

[48] I.-S. Chen, T. N. Jackson, and C. R. Wronski, "Characterization of semiconductor heterojunctions using internal photoemission," Journal of Applied Physics, vol. 79, no. 11, pp. 8470-8474, 1996.

[49] V. V. Afanas'ev, S. Shamuilia, M. Badylevich et al., "Electronic structure of silicon interfaces with amorphous and epitaxial insulating oxides: $\mathrm{Sc}_{2} \mathrm{O}_{3}, \mathrm{Lu}_{2} \mathrm{O}_{3}, \mathrm{LaLuO}_{3}$," Microelectronic Engineering, vol. 84, no. 9-10, pp. 2278-2281, 2007.

[50] T. H. DiStefano, "Field-dependent internal photoemission probe of electronic structure of $\mathrm{Si}_{-} \mathrm{SiO}_{2}$ interface," Journal of Vacuum Science and Technology, vol. 13, no. 4, pp. 856-859, 1976.

[51] V. V. Afanas'ev, M. Houssa, A. Stesmans, and M. M. Heyns, "Electron energy barriers between (100)Si and ultrathin stacks of $\mathrm{SiO}_{2}, \mathrm{Al}_{2} \mathrm{O}_{3}$, and $\mathrm{ZrO}_{2}$ insulators," Applied Physics Letters, vol. 78, no. 20, pp. 3073-3075, 2001.
[52] V. V. Afanas'ev and A. Stesmans, "Internal photoemission over high-k insulating barriers," in Physics and Technology of High-k Gate Dielectrics II-Proceedings of the Intenational Symposium on High Dielectric Constant Materials: Materials Science, Processing, Reliability, and Manufacturing Issues, pp. 115-129, October 2003.

[53] V. V. Afanas'ev, M. Houssa, A. Stesmans, G. J. Adriaenssens, and M. M. Heyns, "Energy barriers between (100)Si and $\mathrm{Al}_{2} \mathrm{O}_{3}$ and $\mathrm{ZrO}_{2}$-based dielectric stacks: internal electron photoemission measurements," Microelectronic Engineering, vol. 59, no. 1-4, pp. 335-339, 2001.

[54] V. V. Afanas'ev, "Comment on "A model for internalphotoemission at high-k oxide/silicon energy barriers',' Journal of Applied Physics, vol. 113, Article ID 166101, 2013.

[55] P. Lange, J. K. Sass, R. Unwin, and D. M. Tench, "Improved analysis of the power law in photoemission yield spectroscopy," Journal of Electroanalytical Chemistry, vol. 122, pp. 387-391, 1981.

[56] P. Lange, D. Grider, H. Neff, J. K. Sass, and R. Unwin, "Limitations of the Fowler method in photoelectric work function determination: oxygen on magnesium single crystal surfaces," Surface Science, vol. 118, no. 1-2, pp. L257-L262, 1982.

[57] V. V. Afanas'ev, A. Stesmans, L. Pantisano et al., " $\mathrm{TiN}_{x} / \mathrm{HfO}_{2}$ interface dipole induced by oxygen scavenging," Applied Physics Letters, vol. 98, no. 13, Article ID 132901, 2011.

[58] H.-Y. Chou, V. V. Afanas'ev, M. Houssa, A. Stesmans, L. Dong, and P. D. Ye, "Electron band alignment at the interface of (100)InSb with atomic-layer deposited $\mathrm{Al}_{2} \mathrm{O}_{3}$, Applied Physics Letters, vol. 101, Article ID 082114, 2012.

[59] V. V. Afanas'ev, A. Stesmans, B. J. Mrstik, and C. Zhao, "Impact of annealing-induced compaction on electronic properties of atomic-layer-deposited $\mathrm{Al}_{2} \mathrm{O}_{3}$," Applied Physics Letters, vol. 81, no. 9, pp. 1678-1680, 2002.

[60] E. P. Gusev, E. Cartier, D. A. Buchanan et al., "Ultrathin high$\mathrm{K}$ metal oxides on silicon: processing, characterization and integration issues," Microelectronic Engineering, vol. 59, no. 1-4, pp. 341-349, 2001.

[61] E. P. Gusev, M. Copel, E. Cartier, I. J. R. Baumvol, C. Krug, and M. A. Gribelyuk, "High-resolution depth profiling in ultrathin $\mathrm{Al}_{2} \mathrm{O}_{3}$ films on Si," Applied Physics Letters, vol. 76, no. 2, pp. 176$178,2000$.

[62] V. V. Afanas'ev, M. Houssa, A. Stesmans, C. Merckling, T. Schram, and J. A. Kittl, "Influence of $\mathrm{Al}_{2} \mathrm{O}_{3}$ crystallization on band offsets at interfaces with $\mathrm{Si}$ and $\mathrm{TiN}_{x}$," Applied Physics Letters, vol. 99, no. 7, Article ID 072103, 2011.

[63] M. Meuris, S. Verhaverbeke, P. W. Mertens et al., "Cleaning technology for improved gate oxide integrity," Microelectronic Engineering, vol. 22, no. 1-4, pp. 21-28, 1993.

[64] B. Ealet, M. H. Elyakhloufi, E. Gillet, and M. Ricci, "Electronic and crystallographic structure of $\gamma$-alumina thin films," Thin Solid Films, vol. 250, no. 1-2, pp. 92-100, 1994.

[65] O. Engström, "A model for internal photoemission at high-k oxide/silicon energy barriers," Journal of Applied Physics, vol. 112, Article ID 064115, 2012.

[66] R. Yan, Q. Zhang, O. A. Kirillov et al., “Graphene as transparent electrode for direct observation of hole photoemission from silicon to oxide," Applied Physics Letters, vol. 102, Article ID 123106, 2013.

[67] V. V. Afanas'ev, A. Stesmans, K. Cherkaoui, and P. K. Hurley, "Electron energy band alignment at the (100)Si/MgO interface," Applied Physics Letters, vol. 96, no. 5, Article ID 052103, 2010. 
[68] V. V. Afanas'ev, M. Houssa, and A. Stesmans, "Electron states at interfaces of semiconductors and metals with insulating films," ECS Transactions, vol. 34, no. 1, pp. 467-472, 2011.

[69] M. Lei, J. H. Yum, J. Price et al., "Spectroscopic evaluation of band alignment of atomic layer deposited $\mathrm{BeO}$ on $\mathrm{Si}(100)$," Applied Physics Letters, vol. 100, no. 12, Article ID 122906, 2012.

[70] J. H. Yum, G. Bersuker, D. A. Ferrer et al., "ALD beryllium oxide: novel barrier layer for high performance gate stacks on $\mathrm{Si}$ and high mobility substrates," in IEEE International Electron Devices Meeting (IEDM '11), p. 28.2, December 2011.

[71] J. H. Yum, T. Akyol, M. Lei et al., "Electrical and physical characteristics for crystalline atomic layer deposited beryllium oxide thin film on Si and GaAs substrates," Thin Solid Films, vol. 520, no. 7, pp. 3091-3095, 2012.

[72] T. H. DiStefano and D. E. Eastman, "The band edge of amorphous $\mathrm{SiO}_{2}$ by photoinjection and photoconductivity measurements," Solid State Communications, vol. 9, no. 24, pp. 2259-2261, 1971.

[73] V. K. Adamchuk and V. V. Afanas'ev, "Determination of the band gap of a thermal oxide on silicon," Soviet Physics: Solid State, vol. 26, pp. 1519-1520, 1984.

[74] R. C. Whited, C. J. Flaten, and W. C. Walker, "Exciton thermoreflectance of $\mathrm{MgO}$ and CaO," Solid State Communications, vol. 13, no. 11, pp. 1903-1905, 1973.

[75] G. Lucovsky, "Correlations between electronic structure of transition metal atoms and performance of high-k gate dielectrics in advanced Si devices," Journal of Non-Crystalline Solids, vol. 303, no. 1, pp. 40-49, 2002.

[76] V. V. Afanas'ev, M. Houssa, and A. Stesmans, "Band alignment at interfaces of oxide insulators with semiconductors," Integrated Ferroelectrics, vol. 125, no. 1, pp. 53-60, 2011.

[77] V. V. Afanas'ev, M. Houssa, and A. Stesmans, "High-k insulating films on semiconductors and metals: general trends in electron band alignment," in High-K Gate Dielectrics for CMOS Technology, G. He and Z. Sun, Eds., chapter 8, Wiley-VCH, Weinheim, Germany, 2012.

[78] V. V. Afanas'ev, A. Stesmans, F. Chen, M. Li, and S. A. Campbell, "Electrical conduction and band offsets in $\mathrm{Si} / \mathrm{Hf}_{x} \mathrm{Ti}_{1-x} \mathrm{O}_{2} /$ metal structures," Journal of Applied Physics, vol. 95, no. 12, pp. 79367939, 2004.

[79] V. V. Afanas'ev, A. Stesmans, C. Zhao, M. Caymax, Z. M. Rittersma, and J. W. Maes, "Band alignment at the interface of (100) Si with $\mathrm{Hf}_{x} \mathrm{Ta}_{1-x} \mathrm{O}_{y}$ high- $\kappa$ dielectric layers," Applied Physics Letters, vol. 86, no. 7, Article ID 072108, pp. 1-3, 2005.

[80] V. V. Afanas'ev, A. Stesmans, C. Zhao, M. Caymax, Z. M. Rittersma, and J. W. Maes, "Band alignment between (1 00 ) Si and Hf-based complex metal oxides," Microelectronic Engineering, vol. 80, pp. 102-105, 2005.

[81] V. V. Afanas'ev, A. Stesmans, F. Chen, X. Shi, and S. A. Campbell, "Internal photoemission of electrons and holes from (100)Si into $\mathrm{HfO}_{2}$," Applied Physics Letters, vol. 81, no. 6, pp. 1053-1055, 2002.

[82] E. E. Hoppe, R. S. Sorbello, and C. R. Aita, "Near-edge optical absorption behavior of sputter deposited hafnium dioxide," Journal of Applied Physics, vol. 101, no. 12, Article ID 123534, 2007.

[83] L. Pantisano, V. V. Afanas'ev, S. Cimino et al., "Towards barrier height modulation in $\mathrm{HfO}_{2} / \mathrm{TiN}$ by oxygen scavengingdielectric defects or metal induced gap states?" Microelectronic Engineering, vol. 88, no. 7, pp. 1251-1254, 2011.
[84] M. Lei, J. H. Yum, S. K. Banerjee, G. Bersuker, and M. C. Downer, "Band offsets of atomic layer deposited $\mathrm{Al}_{2} \mathrm{O}_{3}$ and $\mathrm{HfO}_{2}$ on $\mathrm{Si}$ measured by linear and nonlinear internal photoemission," Physica Status Solidi B, vol. 249, p. 1160, 2012.

[85] V. V. Afanas'ev, A. Stesmans, and W. Tsai, "Determination of interface energy band diagram between (100)Si and mixed Al-Hf oxides using internal electron photoemission," Applied Physics Letters, vol. 82, no. 2, pp. 245-247, 2003.

[86] E. E. Hoppe and C. R. Aita, "Suppression of near-edge optical absorption band in sputter deposited $\mathrm{HfO}_{2}-\mathrm{Al}_{2} \mathrm{O}_{3}$ nanolaminates containing nonmonoclinic $\mathrm{HfO}_{2}$," Applied Physics Letters, vol. 92, no. 26, Article ID 141912, 2008.

[87] F. Amy, A. S. Wan, A. Kahn, F. J. Walker, and R. A. McKee, "Band offsets at heterojunctions between $\mathrm{SrTiO}_{3}$ and $\mathrm{BaTiO}_{3}$ and Si(100)," Journal of Applied Physics, vol. 96, no. 3, pp. 16351639, 2004.

[88] J. C. Maxwell, A Treatise on Electricity and Magnetism, Dover, New York, NY, USA, 1954.

[89] M. Kleefstra and G. C. Herman, "Influence of the image force on the band gap in semiconductors and insulators," Journal of Applied Physics, vol. 51, no. 9, pp. 4923-4926, 1980.

[90] A. G. O'Neill, “Calculation of the image potential in multiple layered structures," Journal of Applied Physics, vol. 58, no. 12, pp. 4740-4742, 1985.

[91] P. de Visschere, "Image forces and MIS Schottky barriers," Solid State Electronics, vol. 29, no. 9, pp. 873-875, 1986.

[92] F. Giustino and A. Pasquarello, "Electronic and dielectric properties of a suboxide interlayer at the silicon-oxide interface in MOS devices," Surface Science, vol. 586, no. 1-3, pp. 183-191, 2005.

[93] S. Markov, P. V. Sushko, S. Roy et al., "Si-SiO ${ }_{2}$ interface bandgap transition-effects on MOS inversion layer," Physica Status Solidi (A) Applications and Materials Science, vol. 205, no. 6, pp. 1290-1295, 2008.

[94] A. Alkauskas, P. Broqvist, F. Devynck, and A. Pasquarello, "Band offsets at semiconductor-oxide interfaces from hybrid density-functional calculations," Physical Review Letters, vol. 101, no. 10, Article ID 106802, 2008.

[95] K. Seino, F. Bechstedt, and P. Kroll, "Band alignment at a nonplanar $\mathrm{Si} / \mathrm{SiO}_{2}$ interface," Physical Review B, vol. 82, no. 8, Article ID 085320, 2010.

[96] W. Mönch, "On the electric-dipole contribution to the valenceband offsets in semiconductor-oxide heterostructures," Applied Physics Letters, vol. 91, no. 4, Article ID 042117, 2007.

[97] V. V. Afanas'ev and A. Stesmans, "Band alignment at the interfaces of $\mathrm{Si}$ and metals with high-permittivity insulating oxides," in High-K Gate Dielectrics, M. Houssa, Ed., pp. 217-250, Institute of Physics Publishing, Bristol, UK, 2003.

[98] A. V. Prokofiev, A. I. Shelykh, and B. T. Melekh, "Periodicity in the band gap variation of $\operatorname{Ln}_{2} \mathrm{X}_{3}(\mathrm{X}=\mathrm{O}, \mathrm{S}, \mathrm{Se})$ in the lanthanide series," Journal of Alloys and Compounds, vol. 242, no. 1-2, pp. 41-44, 1996.

[99] A. I. Shelykh, A. V. Prokofiev, and B. T. Melekh, "Characteristics of the modification in the width of forbidden zone in $\mathrm{Ln}_{2} \mathrm{X}_{3}$ compounds where $(\mathrm{Ln}=\mathrm{REE}, \mathrm{X}=\mathrm{O}, \mathrm{S}, \mathrm{Se})$," Fizika Tverdogo Tela, vol. 38, p. 427, 1996.

[100] A. V. Prokof'ev, A. I. Shelykh, I. A. Smirnov, B. T. Melekh, and W. Assmus, "Absorption edge in rare-earth-doped zirconium dioxide crystals," Physics of the Solid State, vol. 38, no. 9, pp. 1502-1504, 1996. 
[101] V. V. Afanas'ev, A. Stesmans, C. Zhao et al., "Band alignment between (100)Si and complex rare earthtransition metal oxides," Applied Physics Letters, vol. 85, no. 24, pp. 5917-5919, 2004.

[102] V. V. Afanas'ev, A. Stesmans, L. F. Edge, D. G. Schlom, T. Heeg, and J. Schubert, "Band alignment between (100) Si and amorphous $\mathrm{LaAlO}_{3}, \mathrm{LaScO}_{3}$, and $\mathrm{Sc}_{2} \mathrm{O}_{3}$ : Atomically abrupt versus interlayer-containing interfaces," Applied Physics Letters, vol. 88, no. 3, Article ID 032104, pp. 1-3, 2006.

[103] V. V. Afanas'ev, H. -Y. Chou, M. Houssa, and A. Stesmans, "Electron band alignment at $\mathrm{Ge}$ /oxide and $\mathrm{A}_{\mathrm{III}} \mathrm{B}_{\mathrm{V}}$ /oxide interfaces from internal photoemission experiments," ECS Transactions, vol. 58, no. 7, pp. 311-331, 2013.

[104] M. Badylevich, S. Shamuilia, V. V. Afanas'ev et al., "Investigation of the electronic structure at interfaces of crystalline and amorphous $\mathrm{Gd}_{2} \mathrm{O}_{3}$ thin layers with silicon substrates of different orientations," Applied Physics Letters, vol. 90, no. 25, Article ID 252101, 2007.

[105] V. V. Afanas'ev, M. Badylevich, A. Stesmans et al., "Band offsets between $\mathrm{Si}$ and epitaxial rare earth sesquioxides $\left(\mathrm{RE}_{2} \mathrm{O}_{3}, \mathrm{RE}\right.$ = La,Nd,Gd,Lu): effect of $4 f$-shell occupancy," Applied Physics Letters, vol. 93, no. 19, Article ID 192105, 2008.

[106] K. Oishi and Y. Matsuo, "Internal photoemission and photoconduction on $\mathrm{GeO}_{2} / \mathrm{Ge}$ films," Thin Solid Films, vol. 274, no. 1-2, pp. 133-137, 1996.

[107] V. V. Afanas'ev and A. Stesmans, "Energy band alignment at the (100) $\mathrm{Ge} / \mathrm{HfO}_{2}$ interface," Applied Physics Letters, vol. 84, no. 13, pp. 2319-2321, 2004.

[108] V. V. Afanas'ev and A. Stesmans, "Spectroscopy of electron states at interfaces of $\left(\begin{array}{lll}1 & 0 & 0\end{array}\right) \mathrm{Ge}$ with high- $\kappa$ insulators," Materials Science in Semiconductor Processing, vol. 9, no. 4-5, pp. 764-771, 2006.

[109] V. V. Afanas'ev, A. Stesmans, A. Delabie, F. Bellenger, M. Houssa, and M. Meuris, "Electronic structure of $\mathrm{GeO}_{2}$-passivated interfaces of (100)Ge with $\mathrm{Al}_{2} \mathrm{O}_{3}$ and $\mathrm{HfO}_{2}$," Applied Physics Letters, vol. 92, no. 2, Article ID 022109, 2008.

[110] Y. Fukuda, Y. Yazaki, Y. Otani, T. Sato, H. Toyota, and T. Ono, "Low-temperature formation of high-quality $\mathrm{GeO}_{2}$ interlayer for high- $\kappa$ gate dielectrics/Ge by electron-cyclotron-resonance plasma techniques," IEEE Transactions on Electron Devices, vol. 57, no. 1, pp. 282-287, 2010.

[111] W. F. Zhang, T. Nishimula, K. Nagashio, K. Kita, and A. Toriumi, "Conduction band offset at $\mathrm{GeO}_{2} / \mathrm{Ge}$ interface dtermined by internal photoemission and charge-corrected X-ray photoelectron spectroscopies," Applied Physics Letters, vol. 102, Article ID 102106, 2013.

[112] V. V. Afanas'ev, S. Shamuilia, A. Stesmans et al., "Electron energy band alignment at interfaces of (100)Ge with rare-earth oxide insulators," Applied Physics Letters, vol. 88, no. 13, Article ID 132111, 2006.

[113] V. V. Afanas'ev, A. Stesmans, G. Mavrou, and A. Dimoulas, "Beneficial effect of $\mathrm{La}$ on band offsets in Ge/high- $\kappa$ insulator structures with $\mathrm{GeO}_{2}$ and $\mathrm{La}_{2} \mathrm{O}_{3}$ interlayers," Applied Physics Letters, vol. 93, no. 10, Article ID 102115, 2008.

[114] V. V. Afanas'ev, A. Stesmans, A. Delabie et al., "Influence of passivating interlayer on $\mathrm{Ge} / \mathrm{HfO}_{2}$ and $\mathrm{Ge} / \mathrm{Al}_{2} \mathrm{O}_{3}$ interface band diagrams," Materials Science in Semiconductor Processing, vol. 11, no. 5, pp. 230-235, 2008.

[115] M. Perego, G. Seguini, G. Scarel, and M. Fanciulli, "X-ray photoelectron spectroscopy study of energy-band alignments of $\mathrm{Lu}_{2} \mathrm{O}_{3}$ on Ge," Surface and Interface Analysis, vol. 38, no. 4, pp. 494-497, 2006.
[116] V. V. Afanas'ev, H.-Y. Chou, M. Houssa et al., "Transitivity of band offsets between semiconductor heterojunctions and oxide insulators," Applied Physics Letters, vol. 99, no. 17, Article ID 172101, 2011.

[117] M. Perego, G. Seguini, and M. Fanciulli, "Energy band alignment of $\mathrm{HfO}_{2}$ on Ge," Journal of Applied Physics, vol. 100, no. 9, Article ID 093718, 2006.

[118] M. Perego, G. Seguini, and M. Fanciulli, "XPS and IPE analysis of $\mathrm{HfO}_{2}$ band alignment with high-mobility semiconductors," Materials Science in Semiconductor Processing, vol. 11, no. 5, pp. 221-225, 2008.

[119] V. V. Afanas'ev, M. Bassler, G. Pensl, M. J. Schulz, and E. S. Von Kamienski, "Band offsets and electronic structure of $\mathrm{SiC} / \mathrm{SiO}_{2}$ interfaces," Journal of Applied Physics, vol. 79, no. 6, pp. 31083114, 1996.

[120] V. V. Afanas'ev, F. Ciobanu, S. Dimitrijev, G. Pensl, and A. Stesmans, "Band alignment and defect states at SiC/oxide interfaces," Journal of Physics Condensed Matter, vol. 16, no. 17, pp. S1839-S1856, 2004.

[121] V. V. Afanas'ev, F. Ciobanu, G. Pensl, and A. Stesmans, "Contributions to the density of interface states in $\mathrm{SiC}$ MOS structures," in Silicon Carbide: Recent Major Advances, W. J. Choyke, H. Matsunami, and G. Pensl, Eds., pp. 344-371, Springer, Berlin, Germany, 2004.

[122] V. V. Afanas'ev and A. Stesmans, "Valence band offset and hole injection at the $4 \mathrm{H}-, 6 \mathrm{H}-\mathrm{SiC} / \mathrm{SiO}_{2}$ interfaces," Applied Physics Letters, vol. 77, no. 13, pp. 2024-2026, 2000.

[123] L. Souriau, V. Terzieva, W. Vandervorst et al., "High Ge content SGOI substrates obtained by the Ge condensation technique: a template for growth of strained epitaxial Ge," Thin Solid Films, vol. 517, no. 1, pp. 23-26, 2008.

[124] L. Souriau, T. Nguyen, E. Augendre et al., "High-hole-mobility silicon germanium on insulator substrates with high crystalline quality obtained by the germanium condensation technique," Journal of the Electrochemical Society, vol. 156, no. 3, pp. H208H213, 2009.

[125] V. V. Afanas'ev, A. Stesmans, L. Souriau, R. Loo, and M. Meuris, "Valence band energy in confined $\mathrm{Si}_{1-x} \mathrm{Ge}_{x}(0.28<\mathrm{x}<0.93)$ layers," Applied Physics Letters, vol. 94, no. 17, Article ID 172106, 2009.

[126] C. Merckling, X. Sun, Y. Shimura et al., "Molecular beam deposition of $\mathrm{Al}_{2} \mathrm{O}_{3}$ on $\mathrm{p}-\mathrm{Ge}(001) / \mathrm{Ge} 0.95 \mathrm{Sn} 0.05$ heterostructure and impact of a Ge-cap interfacial layer," Applied Physics Letters, vol. 98, no. 19, Article ID 192110, 2011.

[127] V. V. Afanas'ev, A. Stesmans, M. Passlack, and N. Medendorp, "Band offsets at the interfaces of $\mathrm{GaAs}(100)$ with $\mathrm{Gd}_{x} \mathrm{Ga}_{0.4-x} \mathrm{O}_{0.6}$ insulators," Applied Physics Letters, vol. 85, no. 4, pp. 597-599, 2004.

[128] V. V. Afanas'ev, A. Stesmans, R. Droopad, M. Passlack, L. F. Edge, and D. G. Schlom, "Electron energy barriers at interfaces of GaAs(100) with $\mathrm{LaAlO}_{3}$ and $\mathrm{Gd}_{2} \mathrm{O}_{3}$," Applied Physics Letters, vol. 89, no. 9, Article ID 092103, 2006.

[129] G. Seguini, M. Perego, S. Spiga, M. Fanciulli, and A. Dimoulas, "Conduction band offset of $\mathrm{HfO}_{2}$ on GaAs," Applied Physics Letters, vol. 91, no. 19, Article ID 192902, 2007.

[130] N. V. Nguyen, O. A. Kirillov, W. Jiang et al., "Band offsets of atomic-layer-deposited $\mathrm{Al}_{2} \mathrm{O}_{3}$ on GaAs and the effects of surface treatment," Applied Physics Letters, vol. 93, no. 8, Article ID 082105, 2008.

[131] V. V. Afanas'ev, M. Badylevich, A. Stesmans et al., "Energy barriers at interfaces of (100)GaAs with atomic layer deposited 
$\mathrm{Al}_{2} \mathrm{O}_{3}$ and $\mathrm{HfO}_{2}$," Applied Physics Letters, vol. 93, no. 21, Article ID 212104, 2008.

[132] C. L. Hinkle, A. M. Sonnet, E. M. Vogel et al., "GaAs interfacial self-cleaning by atomic layer deposition," Applied Physics Letters, vol. 92, no. 7, Article ID 071901, 2008.

[133] C. L. Hinkle, E. M. Vogel, P. D. Ye, and R. M. Wallace, "Interfacial chemistry of oxides on $\operatorname{In}_{x} \mathrm{Ga}_{(1-x)}$ As and implications for MOSFET applications," Current Opinion in Solid State and Materials Science, vol. 15, no. 5, pp. 188-207, 2011.

[134] H. Y. Chou, E. O’Connor, P. K. Hurley et al., "Interface barriers at the interfaces of polar $\mathrm{GaAs}(111)$ faces with $\mathrm{Al}_{2} \mathrm{O}_{3}$," Applied Physics Letters, vol. 100, no. 14, Article ID 141602, 2012.

[135] V. V. Afanas'ev, A. Stesmans, G. Brammertz et al., "Energy barriers at interfaces between (100) $\mathrm{In}_{x} \mathrm{Ga}_{1-x} \mathrm{As}(0 \leq \mathrm{x} \leq 0.53)$ and atomic-layer deposited $\mathrm{Al}_{2} \mathrm{O}_{3}$ and $\mathrm{HfO}_{2}$," Applied Physics Letters, vol. 94, no. 20, Article ID 202110, 2009.

[136] V. V. Afanas'ev, A. Stesmans, G. Brammertz et al., "Band offsets at interfaces of (1 000$) \operatorname{In}_{x} \mathrm{Ga}_{1-x}$ As $(0 \leq \mathrm{x} \leq 0.53)$ with $\mathrm{Al}_{2} \mathrm{O}_{3}$ and $\mathrm{HfO}_{2}$," Microelectronic Engineering, vol. 86, no. 7-9, pp. 1550$1553,2009$.

[137] N. V. Nguyen, M. Xu, O. A. Kirillov et al., "Band offsets of $\mathrm{Al}_{2} \mathrm{O}_{3} / \mathrm{In}_{x} \mathrm{Ga}_{1-x}$ As ( $\mathrm{x}=0.53$ and 0.75 ) and the effects of postdeposition annealing," Applied Physics Letters, vol. 96, no. 5, Article ID 052107, 2010.

[138] T. J. Kim, J. J. Yoon, S. Y. Hwang et al., "InAs critical-point energies at $22 \mathrm{~K}$ from spectroscopic ellipsometry," Applied Physics Letters, vol. 97, no. 17, Article ID 171912, 2010.

[139] Q. Zhang, G. Zhou, H. G. Xing et al., "Tunnel field-effect transistor heterojunction band alignment by internal photoemission spectroscopy," Applied Physics Letters, vol. 100, no. 10, Article ID 102104, 2012.

[140] Q. Zhang, R. Li, R. Yan et al., "A unique photoemission method to measure semiconductor heterojunction band offsets," Applied Physics Letters, vol. 102, Article ID 012101, 2013.

[141] H.-Y. Chou, V. V. Afanas'ev, A. Stesmans, H. C. Lin, P. K. Hurley, and S. B. Newcomb, "Electron band alignment between (100)InP and atomic-layer deposited $\mathrm{Al}_{2} \mathrm{O}_{3}$," Applied Physics Letters, vol. 97, no. 13, Article ID 132112, 2010.

[142] D. E. Aspnes and A. A. Studna, "Dielectric functions and optical parameters of $\mathrm{Si}$, Ge, GaP, GaAs, GaSb, InP, InAs, and InSb from 1.5 to 6.0 eV," Physical Review B, vol. 27, no. 2, pp. 985-1009, 1983.

[143] V. I. Gavrilenko, A. M. Grekhov, D. V. Korbutyak, and V. G. Litovchenko, Optical Properties of Semiconductors, Naukova Dumka, Kiev, Ukraine, 1987.

[144] V. V. Afanas'ev, H.-Y. Chou, A. Stesmans, C. Merckling, and X. Sun, "Electron band alignment at the interface of (100) GaSb with molecular-beam deposited $\mathrm{Al}_{2} \mathrm{O}_{3}$," Applied Physics Letters, vol. 98, no. 7, Article ID 072102, 2011.

[145] V. V. Afanas'ev, H.-Y. Chou, A. Stesmans, C. Merckling, and $\mathrm{X}$. Sun, "Band offsets at the $\left(\begin{array}{lll}1 & 0 & 0\end{array}\right) \mathrm{GaSb} / \mathrm{Al}_{2} \mathrm{O}_{3}$ interface from internal electron photoemission study," Microelectronic Engineering, vol. 88, no. 7, pp. 1050-1053, 2011.

[146] V. V. Afanas'ev and A. Stesmans, "Barrier characterization at interfaces of high-mobility semiconductors with oxide insulators," ECS Transactions, vol. 25, no. 6, pp. 95-1000, 2009.

[147] G. Pourtois, M. Houssa, B. De Jaeger et al., "Threshold voltage shifts in Si passivated (100)Ge p-channel field effect transistors: insights from first-principles modeling," Applied Physics Letters, vol. 91, no. 2, Article ID 023506, 2007.

[148] Z. Yuan, A. Nainani, B. R. Bennett, J. Brad Boos, M. G. Ancona, and K. C. Saraswat, "Amelioration of interface state response using band engineering in III-V quantum well metal-oxidesemiconductor field-effect transistors," Applied Physics Letters, vol. 100, no. 14, Article ID 143503, 2012.

[149] H. Zhao, Y.-T. Chen, J. H. Yum et al., "Effects of barrier layers on device performance of high mobility $\mathrm{In}_{0.7} \mathrm{Ga}_{0.3}$ as metal-oxidesemiconductor field-effect-transistors," Applied Physics Letters, vol. 96, no. 10, Article ID 102101, 2010.

[150] M. G. Ancona, B. R. Bennett, and J. B. Boos, "Scaling projections for Sb-based p-channel FETs," Solid-State Electronics, vol. 54, no. 11, pp. 1349-1358, 2010.

[151] A. C. Seabaugh and Q. Zhang, "Low-voltage tunnel transistors for beyond CMOS logic," Proceedings of the IEEE, vol. 98, no. 12, pp. 2095-2110, 2010.

[152] S. Adachi, "Model dielectric constants of Si and Ge," Physical Review B, vol. 38, no. 18, pp. 12966-12976, 1988.

[153] R. T. Tung, "Recent advances in Schottky barrier concepts," Materials Science and Engineering: $R$, vol. 35, no. 1-3, pp. 1-138, 2001. 

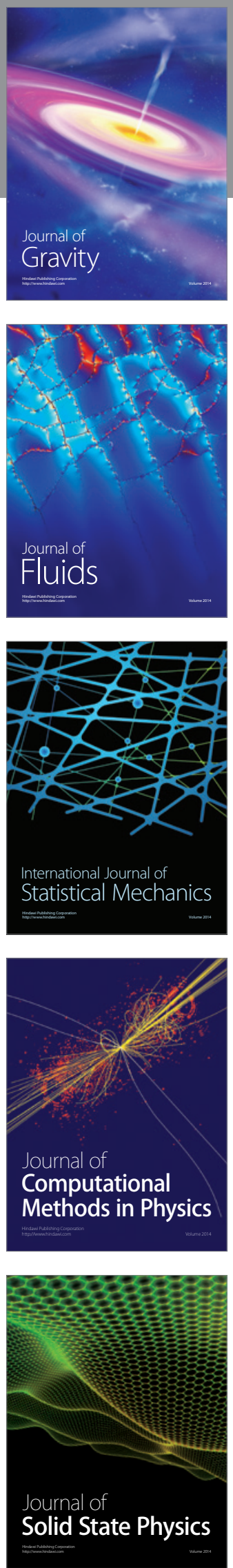

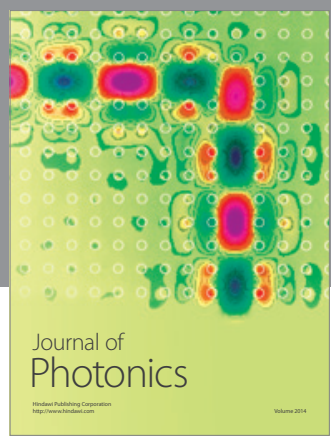

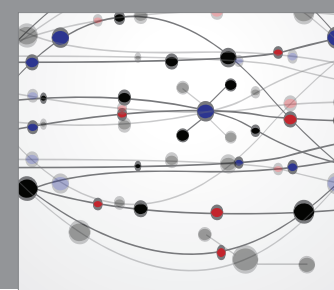

The Scientific World Journal

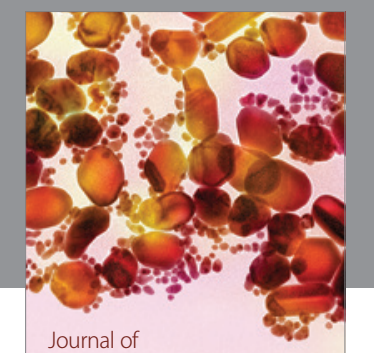

Soft Matter
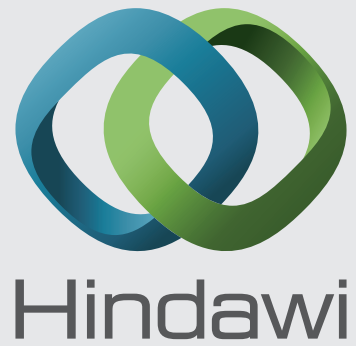

Submit your manuscripts at

http://www.hindawi.com
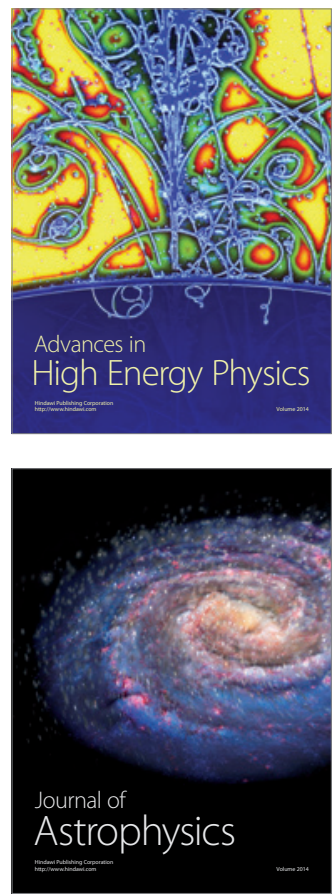
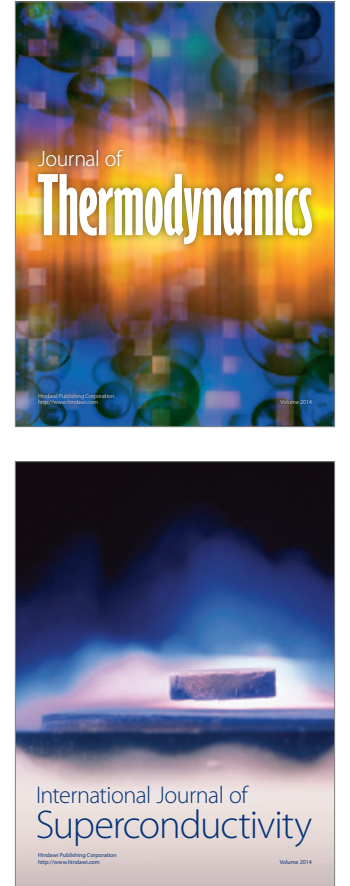
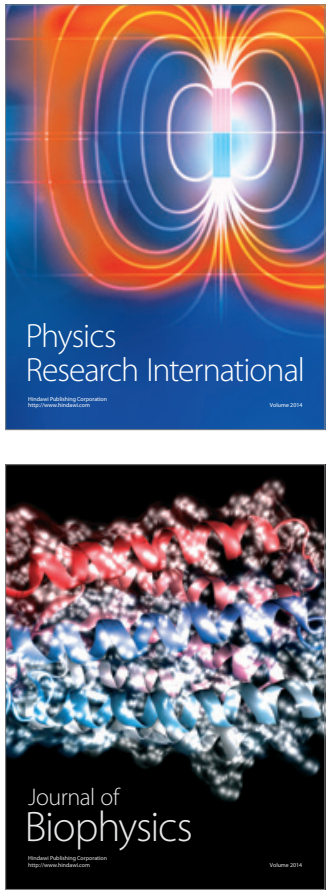
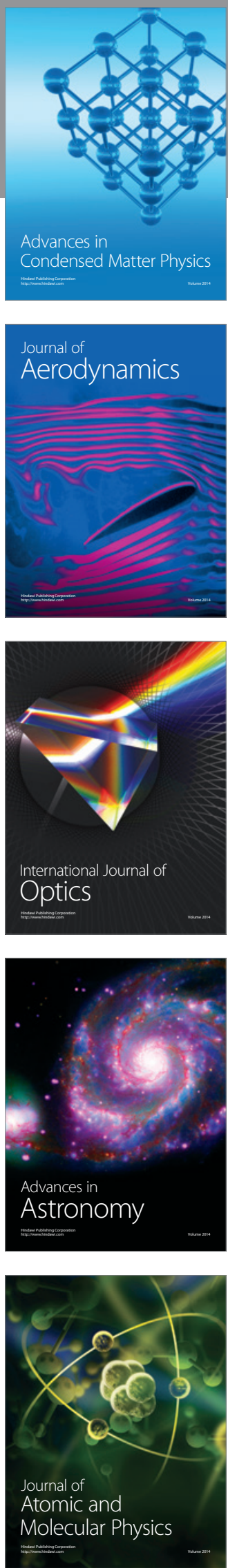